\title{
The Search for Superconductivity in High Pressure Hydrides
}

\author{
Tiange Bi, Niloofar Zarifi, Tyson Terpstra, Eva Zurek* \\ Department of Chemistry \\ University at Buffalo \\ State University of New York \\ Buffalo, NY 14260-3000, USA
}

tiangebi@buffalo.edu,nzarifi@buffalo.edu, tysonter@buffalo.edu, *ezurek@buffalo.edu

June 4, 2018

\begin{abstract}
The computational and experimental exploration of the phase diagrams of binary hydrides under high pressure has uncovered phases with novel stoichiometries and structures, some which are superconducting at quite high temperatures. Herein we review the plethora of studies that have been undertaken in the last decade on the main group and transition metal hydrides, as well as a few of the rare earth hydrides at pressures attainable in diamond anvil cells. The aggregate of data shows that the propensity for superconductivity is dependent upon the species used to "dope" hydrogen, with some of the highest values obtained for elements that belong to the alkaline and rare earth, or the pnictogen and chalcogen families.
\end{abstract}

\section{Keywords:}

superconductivity, hydrides, high pressure, density functional theory, electronic structure, crystal structure prediction, materials, extreme conditions, metallic hydrogen, Bardeen-Cooper-Schrieffer superconductor 


\section{Introduction}

The metalization of hydrogen under pressure was first proposed by J. D. Bernal, but only later transcribed by Wigner and Huntington in their seminal 1935 paper, which discussed the possibility of hydrogen transforming to an alkali metal-like monoatomic solid at $P>25 \mathrm{GPa}{ }^{1} \mathrm{In}$ 1968 Ashcroft predicted that this elusive substance had the propensity to be a high temperature Bardeen-Cooper-Schrieffer (BCS) type superconductor based upon its large density of states at the Fermi level, high phonon frequencies (a result of the small atomic mass), and substantial electron-phonon coupling (due to the lack of core electrons and strong covalent bonding). ${ }^{2}$ Nearly 40 years later the same considerations led Ashcroft to propose that hydrogen-rich solids, such as the group 14 hydrides, had the potential to become high temperature superconductors under pressure. ${ }^{3 / 4}$ Moreover, he speculated that the addition of a second element to hydrogen could reduce the physical pressure required to metallize the system via doping ${ }^{[5}$ or "chemical precompression". 3 Herein, we provide a thorough review of the efforts, both experimental and theoretical, undertaken to metallize hydrogen-rich solids under pressure in the search for new superconducting materials.

Pressure can coerce compounds to assume stoichiometries and geometric arrangements that would not be accessible at atmospheric conditions. ${ }^{6-17}$ Because experimental trial-and-error high pressure syntheses can be expensive to carry out and the results difficult to analyze, it is desirable to predict which elemental combinations and pressures could be used to synthesize compounds with useful properties. However, neither chemical intuition nor data-mining techniques are typically useful for these purposes because they have been developed based upon information gathered at atmospheric conditions. Fortunately, the spectacular advances in computer hardware, coupled with the developments in a priori crystal structure prediction (CSP) using Density Functional Theory (DFT) ${ }^{18}$ has led to synergy between experiment and theory in high pressure research. 1525 -28 Computations are carried out to predict the structures and properties of targets for synthesis, and also to aid in the characterization of phases that have been made in experiment. This synergy has been instrumental in advancing the research carried out on high pressure hydrides.

Ashcroft's original predictions regarding superconductivity in hydrogen-rich systems ${ }^{3 / 4}$ have led to a plethora of theoretical, and some experimental investigations of high pressure hydrides. By now the phase diagrams of most binary hydrides have been explored on a computer. In the early days of CSP it was common to interrogate the stability of a single stoichiometry by carrying out geometry optimizations, as a function of pressure, on simple lattices or crystal structure types that were known for other chemically similar systems. The next major step in CSP was taken when it became standard to employ automated techniques such as random structure searches, 27/29

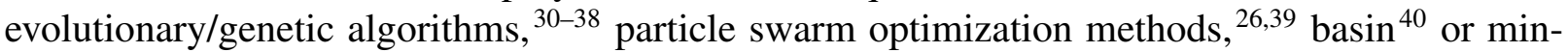
ima hopping, ${ }^{41}$ metadynamics ${ }^{42}$ and simulated annealing ${ }^{43}$ to predict the global minima at a given set of conditions. However, these studies were typically limited in that they investigated the stoichiometries that were known to be stable at atmospheric conditions (e.g. $\mathrm{H}_{2} \mathrm{~S}$ ). It was eventually realized that under pressure the stable and metastable structures may have very different stoichiometries, and by now it is common practice to use CSP to predict the thermodynamically and dynamically stable phases while varying the hydrogen content (e.g. $\mathrm{H}_{n} \mathrm{~S}$ for a range of $n$ ) as a function of pressure. In fact, there is by now a standard "recipe" of how to carry out these 
studies. However, because of the stochastic nature of CSP searches the only way to be certain that the global minimum has been found is to compute all of the local minima (which is impractical for all but the simplest systems), and because different decisions can be made about which pressures, stoichiometries and unit cell sizes are considered in the CSP searches, it should not be surprising that the findings of two or more investigations on the same system can, at times, yield different results. Moreover, it is not always clear whether or not the phases that are formed in experiment are the global minima or simply metastable. For example, recent work on a sample of compressed hydrogen disulfide has shown that the stoichiometries that are formed, and their $T_{c}$, depends upon the experimental conditions. $\underline{\text {.44 }}$

Therefore, this review compiles and presents all of the theoretical and much of the experimental data available to date on the high pressure investigations of binary hydrides, with a particular emphasis on the structures that have been proposed as being stable and their propensity for superconductivity (in a few cases the main motivation for the study may have been for other applications such as hydrogen storage ${ }^{7}$ ). The focus is on hydrides of the main group elements, the transition metal elements, and only a few of the rare earths (Sc, Y, La) are discussed in detail, typically at pressures that can be achieved reliably in a diamond anvil cell (DAC), $400 \mathrm{GPa}$. The results are organized according to the groups within the periodic table to which the "dopant" element added to hydrogen belongs. We note that a number of excellent reviews of the high pressure hydrides have appeared recently, however most of them have focused on particular elements or authors, and none have presented a thorough compilation of the work carried out so far. $25 / 28 / 45,50$

We hope this review is therefore useful for those interested in comparing the results obtained in different studies of the same set of hydrides, for determining which binary compounds have not yet been intensely studied, and for unveiling chemical trends in the properties and behavior of the binary hydrides under pressure. We also point the reader to a number of reviews that cover advances in high pressure CSP, 25 the successes and limitations of DFT calculations in high pressure research, $\frac{15}{15}$ as well as the methods employed to estimate $T_{c}$ in BCS-type superconductors. $\frac{51}{1}$

\section{Group 1: Alkali Metal Hydrides}

At atmospheric conditions the alkali metal $(\mathrm{M}=\mathrm{Li}, \mathrm{Na}, \mathrm{K}, \mathrm{Rb}, \mathrm{Cs})$ hydrides adopt an $\mathrm{MH}$ stoichiometry and crystallize in the rock-salt $(B 1)$ structure. The band gaps of these ionic solids are large, ranging from 4- $6 \mathrm{eV} . \underline{5253}$ A number of phase changes $(B 1 \rightarrow B 2$ for $\mathrm{M}=\mathrm{Na}, \mathrm{K}, \mathrm{Rb}, \mathrm{Cs}$; $B 2 \rightarrow \mathrm{CrB}$ for $\mathrm{M}=\mathrm{Rb}, \mathrm{Cs}$ ) occur at progressively lower pressures for the heavier alkali metals. ${ }^{54}$ Computations suggest that under sufficient compression $\mathrm{KH}$ will also assume the $\mathrm{CrB}$ structure. ${ }^{5859}$ A hitherto unobserved transition to a $P n m a$ and a $P 6_{3} / m m c$ phase was computationally predicted in $\mathrm{CsH}, \stackrel{60}{6}$ but it has not yet been observed. A pressure induced insulator to metal transition is likely to take place in these systems due to pressure induced broadening of the valence and conduction bands. So far these alkali hydrides have not been metalized, but extrapolation of experimentally determined band gaps and first-principles calculations suggests that band gap closure may occur between 300-1000 GPa. ${ }^{57,61 / 62}$ Because metalization in these systems will likely occur because of pressure induced band overlap, the "classic" alkali hydrides are unlikely to have a high density of states (DOS) at the Fermi level $\left(E_{F}\right)$, and therefore will not be good 
candidates for high temperature superconductivity at pressures attainable in a DAC.

The only alkali metal subhydrides that were predicted to become stable under pressure are $\mathrm{LiH}_{m}$ with $m>1 .{ }^{63}$ They were computed to be thermodynamically stable with respect to decomposition into $\mathrm{Li}$ and $\mathrm{LiH}$ in a very narrow pressure range $(\sim 50-100 \mathrm{GPa})$. None of the phases found were good metals, but the band structure of two $\mathrm{Li}_{5} \mathrm{H}$ compounds that had the lowest enthalpies of formation, $\Delta H_{F}$, contained two conical bands with a linear dispersion around $E_{F}$. Thus, even though these phases are not promising for superconductivity their electronic structure features a Dirac cone, hinting that they may have unusual electron transport properties.

Evolutionary algorithms coupled with the PBE functional predicted that phases with stoichiometries such as $\mathrm{LiH}_{2}, \mathrm{LiH}_{6}$ and $\mathrm{LiH}_{8}$ would become stable at pressures ranging from $\sim 100$ $300 \mathrm{GPa} \cdot{ }^{64}$ Fig. 1 (a) plots $\Delta H_{F}$ for the reaction $\mathrm{LiH}+\left(\frac{n-1}{2}\right) \mathrm{H}_{2} \rightarrow \mathrm{LiH}_{n}$ versus the mole fraction of hydrogen in the products. The solid lines denote the convex hulls for the different pressures. Any phase whose $\Delta H_{F}$ lies on the hull is thermodynamically stable, whereas other phases may be metastable. $\mathrm{LiH}_{2}$, shown in Fig. 1 (b), was comprised of $\mathrm{H}_{2}$ and $\mathrm{H}^{-}$units and it was found to metallize via pressure induced broadening and overlap of the $\mathrm{H}^{-}$donor/impurity band with the $\mathrm{H}_{2} \sigma^{*}$ anti-bonding band. The DOS at $E_{F}$ was low, and the $T_{c}$ of $\mathrm{LiH}_{2}$ was calculated to be $0 \mathrm{~K}$ at $150 \mathrm{GPa} .{ }^{65]}$ The most stable $\mathrm{LiH}_{6}$ (shown in Fig. 1.(b)) and $\mathrm{LiH}_{8}$ phases, on the other hand, only contained molecular hydrogen units that obtained a partial negative charge via electron transfer from the electropositive lithium atom, i.e. $\mathrm{H}_{2}^{\delta-}$. These systems were good metals because of the partial filling of the $\mathrm{H}_{2} \sigma^{*}$ anti-bonding bands even at $1 \mathrm{~atm}$. The high DOS at $E_{F}$ persisted at pressures where they became stable, wherein DFT calculations predicted $T_{c}$ values that ranged from $\sim 30-80 \mathrm{~K} .65$

Recently, Pépin and co-workers succeeded in synthesizing the lithium polyhydrides after squeezing $\mathrm{LiH}$ in a DAC at $300 \mathrm{~K}$ above $130 \mathrm{GPa} .{ }^{66}$ Synchrotron infrared (IR) absorption revealed peaks whose frequencies differed significantly from the $\mathrm{H}-\mathrm{H}$ stretching mode in pure $\mathrm{H}_{2}$ (the $\mathrm{H}_{2}$ vibron), but roughly matched those computed for the $\mathrm{LiH}_{2}$ and $\mathrm{LiH}_{6}$ phases in $\mathrm{Ref} . \underline{64}$ Therefore, Pépin et al. proposed that lithium diffuses into the diamond where it can react with carbon, and this mechanism leads to the formation of an $\mathrm{LiH}_{6}$ layer at the diamond/sample interface, and an $\mathrm{LiH}_{2}$ layer at the $\mathrm{LiH}_{6} / \mathrm{LiH}$ interface. Further characterization was not possible, however it was noted that the sample remained optically transparent until $215 \mathrm{GPa}$, and the IR measurements did not provide any evidence of metallicity. This suggests that despite the agreement of the measured and computed IR data, the $\mathrm{LiH}_{6}$ phase predicted in Ref. ${ }^{64}$ cannot be formed in experiment because it must be metallic as a consequence of the fact that its unit cell contains an odd number of electrons.

The discrepancy between theory and experiment inspired a theoretical study that employed the nonlocal van der Waals (vdW) including functional of Dion et al. (vdW-DF), ${ }^{67}$ which incorporates the effects of dispersion self-consistently. $\frac{68}{\text { It }}$ was shown that the inclusion of vdW interactions affects the relative stabilities of the polyhydride phases, so that the $\mathrm{LiH}_{6}$ phase predicted to be

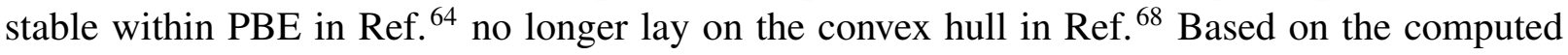
enthalpies, the insulating character observed in experiment, plus a comparison of the experimental and theoretical vibron frequencies as a function of pressure, the authors of Ref. ${ }^{[6]}$ concluded that $\mathrm{LiH}_{2}, \mathrm{LiH}_{9}$ or a metastable $\mathrm{LiH}_{7}$ phase are the most likely candidates for the species made in experiment. Exploratory calculations indicated that vdW interactions did not influence the structures nor stabilities of the heavier group I polyhydrides. 
(a) $100 \%$ LiH $\quad \mathrm{n}=2 \quad 3 \quad 4 \quad 5678 \quad 100 \% \mathrm{H}_{2}$ (b)

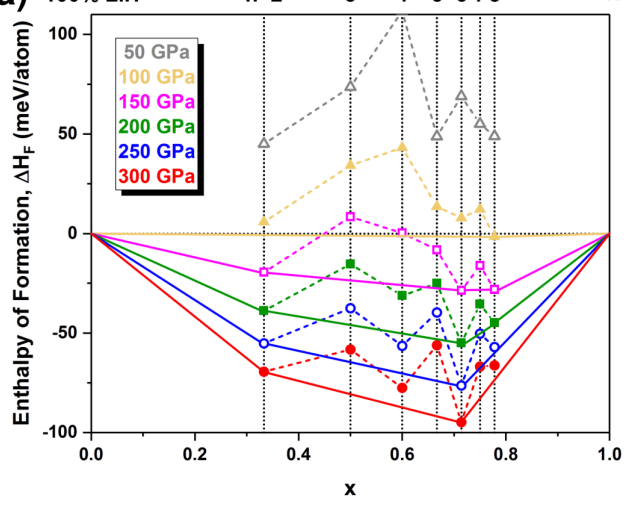

(c)

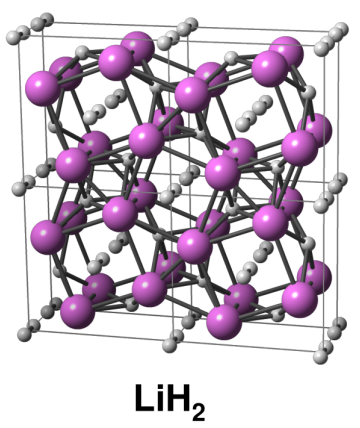

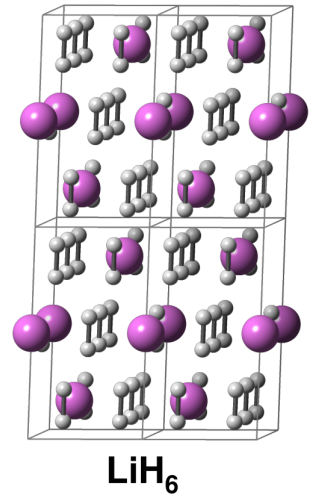

Figure 1: (a) Enthalpies of formation, $\Delta H_{F}$, with respect to $\mathrm{LiH}$ and $\mathrm{H}_{2}$ of the most stable $\mathrm{LiH}_{n}$, $n=1-8$, phases predicted in Ref. ${ }^{64}$ between 50-300 GPa. The $x$ axis shows the fraction of $\mathrm{H}_{2}$ in the structures and the solid lines denote the convex hulls. Supercells of the (b) $P 4 / m b m-\mathrm{LiH}_{2}$ and (c) $R \overline{3} m-\mathrm{LiH}_{6}$ phases that fell on the hull between 100-300 GPa are shown.

A theoretical investigation predicting that sodium polyhydrides will become stable above $\sim 25 \mathrm{GPa}^{69}$ inspired an experimental study where these phases were synthesized in a DAC above $40 \mathrm{GPa}$ and $2000 \mathrm{~K} \cdot{ }^{[0]}$ Because the computed X-ray diffraction (XRD) patterns and Raman spectra of the phases predicted as being stable in Ref. ${ }^{69}$ could not account for the experimental results, further CSP searches were carried out in Ref. ${ }^{70}$ on a larger range of stoichiometries than those originally considered. An $\mathrm{NaH}_{3}$ phase was found to be the lowest point on the convex hull at $50 \mathrm{GPa}$, and the observables calculated for the $\mathrm{NaH}_{3}$ and $\mathrm{NaH}_{7}$ stoichiometries gave the best agreement with experiment. The $\mathrm{NaH}_{3}$ and $\mathrm{NaH}_{7}$ phases were insulating at $50 \mathrm{GPa}$, and they were comprised of $\mathrm{H}_{2}$ as well as $\mathrm{H}^{-}$and $\mathrm{H}_{3}^{-}$units, respectively.

Ref. ${ }^{71}$ used CSP to find the most stable structures of $\mathrm{KH}_{n}$ with $n=2,4,6,8$. Calculations were carried out to determine the superconducting properties of a $C 2 / c-\mathrm{KH}_{6}$ phase with a large DOS at $E_{F}$ that was comprised of $\mathrm{H}_{2}$ units. The $T_{c}$ was estimated to be $58-70 \mathrm{~K}$ at $230 \mathrm{GPa}$ and 46-57 K at $300 \mathrm{GPa}$. Another study carried out evolutionary searches on $\mathrm{KH}_{n}$ with $n=2-13$ and found that phases with the $\mathrm{KH}_{6}$ stoichiometry did not lie on the convex hull up to pressures of $250 \mathrm{GPa} \cdot{ }^{72}$ Instead, the systems that were thermodynamically and dynamically stable contained $\mathrm{H}^{-}$and $\mathrm{H}_{3}^{-}$units, and they were unlikely to be good superconductors.

Finally, CSP has been employed to predict the structures of the polyhydrides of rubidium ${ }^{73}$ and cesium. ${ }^{74}$ Above $30 \mathrm{GPa}$ the rubidium polyhydride with the most negative $\Delta H_{F}$ was $\mathrm{RbH}_{5}$, which was comprised of linear $\mathrm{H}_{3}^{-}$and $\mathrm{H}_{2}$ units. The lowest point on the $\mathrm{CsH}_{n}$ convex hulls between 50-100 $\mathrm{GPa}$ was $\mathrm{CsH}_{3}$, and five nearly isoenthalpic structures were found. They were all comprised of linear $\mathrm{H}_{3}^{-}$building blocks and $\mathrm{Cs}^{+}$ions whose positions were related to those of the silicon and thorium atoms, respectively, in either $\alpha$ - or $\beta-\mathrm{ThSi}_{2}$. Metalization of $\mathrm{RbH}_{5}$ and $\mathrm{CsH}_{3}$ was predicted to occur at high pressures due to pressure induced band overlap of the $\mathrm{H}_{3}^{-}$ non-bonding bands with either the metal $d$-bands or the $\mathrm{H}_{3}^{-}$anti-bonding bands, but the DOS at $E_{F}$ was low suggesting that these systems are not good candidates for superconductivity.

Most of the thermodynamically and dynamically stable polyhydrides of the alkali metals 
that were predicted via CSP contained $\mathrm{H}^{-}$and $\mathrm{H}_{3}^{-}$units rendering them insulating at low pressures. Metalization could be induced via pressure induced broadening and overlap of the $\mathrm{H}^{-}$ donor/impurity band with the $\mathrm{H}_{2} \sigma^{*}$ anti-bonding bands, or the $\mathrm{H}_{3}^{-}$non-bonding and anti-bonding bands. As a result these phases had a low DOS at $E_{F}$, suggesting that their $T_{c}$ values were likely

to be low, like what was found for $\mathrm{LiH}_{2} \cdot{ }^{65}$ Phases whose hydrogenic lattices only contained $\mathrm{H}_{2}^{\delta-}$ molecules had a substantial DOS at $E_{F}$ suggesting they could have a high $T_{c}$. Calculations predicted $T_{c}$ values ranging from $\sim 30-80 \mathrm{~K}$ for $\mathrm{LiH}_{6}, \mathrm{LiH}_{8}$, and $\mathrm{KH}_{6}$. However, it is likely that these phases are metastable.

\section{Group 2: Alkaline Earth Metal Hydrides}

Whereas $\mathrm{BeH}_{2}$ and $\mathrm{MgH}_{2}$ undergo a unique sequence of phase transitions under pressure, $\mathrm{CaH}_{2},{ }^{80 \mid 81} \mathrm{SrH}_{2}{ }^{82}$ and $\mathrm{BaH}_{2}{ }^{83 / 84}$ all undergo the same structural changes, but they occur at lower pressures for the heavier alkaline earth metals. Despite their large band gaps at atmospheric conditions, these phases are computed, within PBE, to metallize at pressures attainable in a DAC. Band gap closure occurs at higher pressures for the lighter systems. ${ }^{[85}$ However, the DOS at $E_{F}$ for the metallic phases is low, and DFT calculations predict small to moderate $T_{c}$ values, for example $38 \mathrm{~K}^{75}$ and $32-44 \mathrm{~K}^{[86}$ for $\mathrm{BeH}_{2}$ at $250 \mathrm{GPa}, 16-23 \mathrm{~K}$ for $\mathrm{MgH}_{2}$ at $180 \mathrm{GPa}, \stackrel{87}{\text { and only }}$ a few $\mathrm{mK}$ for $\mathrm{BaH}_{2}$ at $60 \mathrm{GPa} . \underline{83}$

CSP techniques have been used to predict the most stable structures of the polyhydrides of magnesium,,$\frac{8788}{}$ calcium, ${ }^{89}$ strontium ${ }^{90191}$ and barium, ${ }^{92}$ i.e. $\mathrm{MH}_{n}$ with $n>2$, under pressure. Similar to what was observed for the alkali metal polyhydrides, thermodynamic stability was achieved at the lowest pressures for the heaviest systems: whereas $\mathrm{BeH}_{2}$ was the only hydride of beryllium that was stable below $200 \mathrm{GPa}$, the polyhydrides of barium were predicted to form above $20 \mathrm{GPa} \cdot{ }^{[22}$ For comparison $\mathrm{LiH}_{n}{ }^{64}$ and $\mathrm{CsH}_{n}{ }^{74}$ with $n>1$ were predicted to stabilize by $\sim 120 \mathrm{GPa}$ and $3 \mathrm{GPa}$, respectively, as a result of the lower ionization potentials of Li and $\mathrm{Cs}$ as compared to those of $\mathrm{Be}$ and $\mathrm{Ba}$. Whereas the hydrogen content in the stoichiometry that had the most negative $\Delta H_{F}$ always increased with increasing pressure for the alkaline earth polyhydrides, this was not always the case for the alkali metal polyhydrides. Another difference, that is important for the $T_{c} \mathrm{~s}$ of these phases, is that whereas the hydrogenic sublattices of the alkali metal polyhydrides only contained discrete hydrogenic motifs $\left(\mathrm{H}^{-}, \mathrm{H}_{2}, \mathrm{H}_{2}^{\delta-}\right.$, and $\left.\mathrm{H}_{3}^{-}\right)$, a few of the stable alkaline earth polyhydrides were comprised of extended hydrogenic lattices such as clathrate-like structures or one-dimensional chains, as shown in Fig. 2(a) and Fig. 2(b). It turns out that these structural motifs are linked to high temperature superconductivity.

In 2012 Wang et al. predicted that the $\mathrm{CaH}_{6}$ phase illustrated in Fig. 2(a) would become thermodynamically and dynamically stable above $150 \mathrm{GPa} .{ }^{[89}$ The calcium atoms in this $\operatorname{Im} \overline{3} \mathrm{~m}$ symmetry phase are found in a body-centered arrangement and the hydrogen atoms encapsulate them in a sodalite-like clathrate cage. DFT calculations indicated a weak bonding interaction between adjacent hydrogen atoms whose $\mathrm{H}-\mathrm{H}$ distances measured $1.24 \AA$. A particularly large electron-phonon-couping (EPC, or $\lambda$ ) parameter of 2.69 was found, with the largest contribution towards $\lambda$ arising from the modes that resulted from the vibrations of the atoms in the $\mathrm{H}_{4}$ faces comprising the clathrate-like lattice. The estimated $T_{c}$ at $150 \mathrm{GPa}$ ranged from $220-235 \mathrm{~K}$ for a Coulomb pseudopotential $\left(\mu^{*}\right)$ value of $0.13-0.1$, and $T_{c}$ decreased under pressure. Because of 
(a)

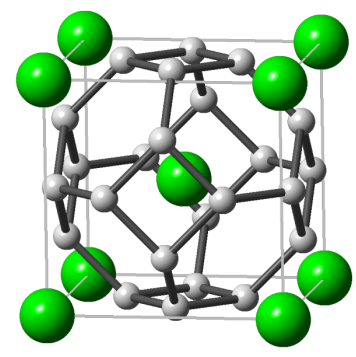

$\mathrm{CaH}_{6}$ (b)

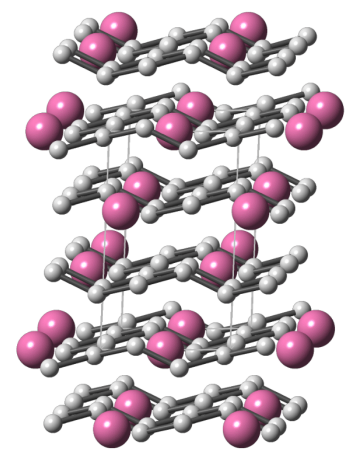

(c)

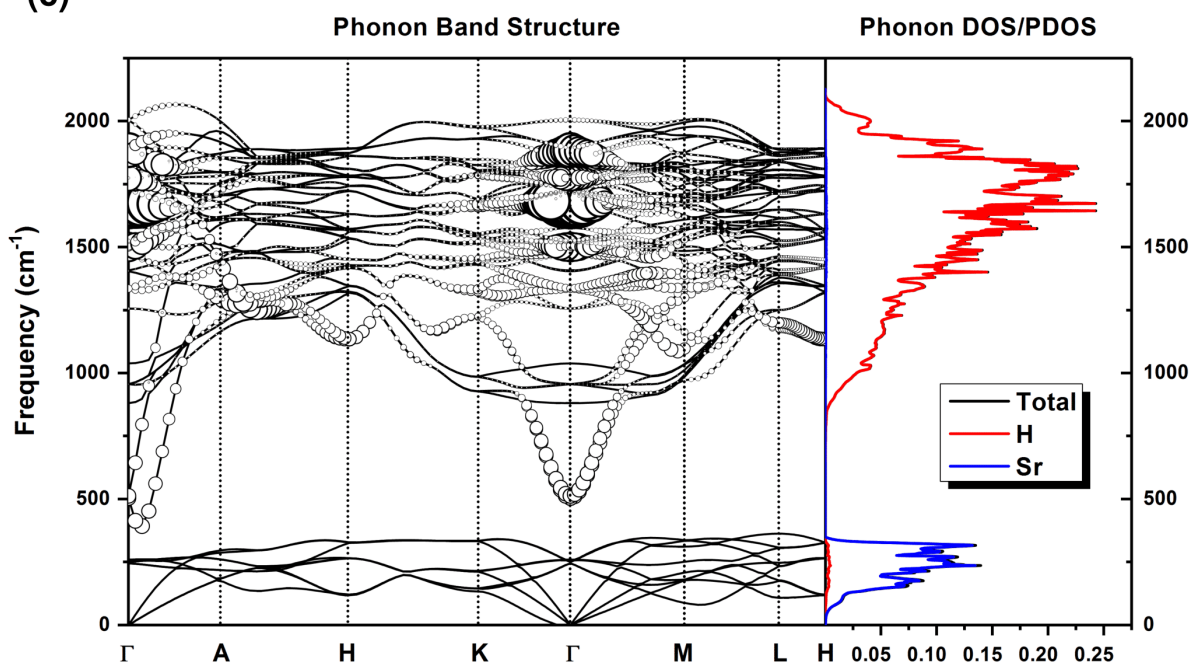

Figure 2: Unit cells of (a) the sodalite-like $\operatorname{Im} \overline{3} m-\mathrm{CaH}_{6}, \underline{89}$ and (b) $R \overline{3} m-\mathrm{SrH}_{6}{ }^{90 \mid 91}$ phases predicted to be stable above $150 \mathrm{GPa}$ and $250 \mathrm{GPa}$, respectively. (c) The calculated phonon band structure along with the phonon projected density of states (PDOS) plot of $R \overline{3} m-\mathrm{SrH}_{6}$ at $250 \mathrm{GPa}$. Circles in the phonon band structure represent the phonon line-width. Because of the separation of the hydrogen-based and strontium-based vibrations, the PDOS overlaps with the total DOS. The calculated values of $\lambda=1.10$, and $\omega_{\log }=1358 \mathrm{~K}$ gave $T_{c}$ values of $108 \mathrm{~K} / 156 \mathrm{~K}$ as estimated using the modified McMillan/Eliashberg equation for $\mu^{*}=0.10$.

the large value of $T_{c}$ predicted for this phase DFT calculations were employed to study $\mathrm{MgH}_{6} .{ }^{88}$ It was shown that this structure, which is isotypic to $I m \overline{3} m-\mathrm{CaH}_{6}$, became stable with respect to decomposition into $\mathrm{MgH}_{2}$ and $\mathrm{H}_{2}$ above $263 \mathrm{GPa}$ when the zero-point-energy (ZPE) was included. However, because CSP searches were not carried out as a function of stoichiometry at this pressure, it is not clear if this $\mathrm{MgH}_{6}$ phase comprises the convex hull, which has been calculated elsewhere. ${ }^{87}$ The electron localization function (ELF) indicated bonding interactions within the hydrogenic framework in $\mathrm{MgH}_{6}$, and a large EPC parameter yielded a $T_{c}$ ranging from 263-271 K between $300-400 \mathrm{GPa} .{ }^{88}$ 
The reason why $I m \overline{3} m-\mathrm{MgH}_{6}$ and $\mathrm{CaH}_{6}$ have such a high $T_{c}$ can be traced back to their large DOS at $E_{F}$, which is derived primarily from hydrogen-like states, and the pronounced impact on the electronic structure that results from the motions of the atoms comprising their hydrogenic lattices. As has been recently pointed out, perturbing the hydrogen atoms within quasi-molecular units does not have such a large impact on the electronic structure of the high pressure phases of the hydrides $25 / 46$ yielding lower $\lambda$, and concomitantly $T_{c}$ values. Therefore, systems with extended hydrogenic lattices are more likely to become superconducting at higher temperatures as compared to those containing $\mathrm{H}^{-}, \mathrm{H}_{2}^{\delta-}$ or $\mathrm{H}_{3}^{-}$units, for example.

Another phase related to the $I m \overline{3} m-\mathrm{CaH}_{6}$ structure is $R \overline{3} m-\mathrm{SrH}_{6}$, which becomes stable near $\sim 250 \mathrm{GPa} \cdot{ }^{90 \mid 91}$ The latter can be derived from the former by elongating four out of the six closest metal-metal contacts and distorting the face that bisects them so it is no longer an ideal hexagon. The elongation of some of the $\mathrm{H}-\mathrm{H}$ contacts leads to the formation of a hydrogenic lattice comprised of one-dimensional helical hydrogenic chains, as illustrated in Fig.2(b). Fig.2(c) provides the results of our calculations of the phonon band structure and phonon projected density of states of $R \overline{3} m-\mathrm{SrH}_{6}$. The EPC of $R \overline{3} m-\mathrm{SrH}_{6}$ is lower than what was reported for $I m \overline{3} m-\mathrm{CaH}_{6}$ and $\mathrm{MgH}_{6}, \lambda=1.10$ at $250 \mathrm{GPa}$, and so is the estimated $T_{c}$.

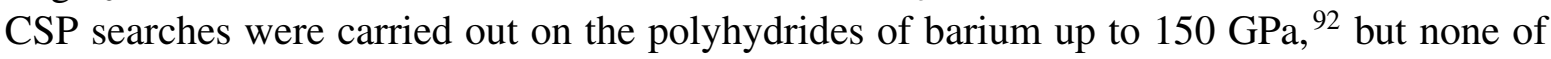
the phases found were isotypic with either $I m \overline{3} m-\mathrm{CaH}_{6}$ or $R \overline{3} m-\mathrm{SrH}_{6}$. Perhaps higher pressures would yield geometries with motifs that are conducive towards high temperature superconductivity within $\mathrm{BaH}_{n}$.

\section{$4 d$-Block Elements}

\subsection{Group 3: Scandium, Yttrium, Lanthanum}

Scandium, yttrium and lanthanum belong to the rare earth (RE) elements, and their chemical behavior resembles that observed in the lanthanides. So, whereas at 1 atm most of the $d$-block elements form hydrides where the $\mathrm{H} / \mathrm{M}$ ratio is less than $1, \mathrm{Sc}, \mathrm{Y}$ and La can form dihydrides that assume a $\mathrm{CaF}_{2}$ type crystal structure where hydrogen fills the tetrahedral holes of a face centered cubic (fcc) lattice, and trihydrides of these elements can be made under pressure. $\mathrm{ScH}_{3}$ and $\mathrm{YH}_{3}$ assume a hexagonal closed packed (hcp) structure. Increasing the pressure leads to a structural transition to an fcc lattice, and this transformation has been studied via DFT calculations. $\underline{93 \mid 94}$ The superconducting behavior of $\mathrm{ScH}_{3},{ }^{95 \mid 96} \mathrm{YH}_{3}{ }^{95 \mid 97}$ and $\mathrm{LaH}_{3}{ }^{95}$ in the fcc structure has been estimated using the Allen-Dynes approximation. The highest $T_{c}$ values calculated, 20, 40, and $18 \mathrm{~K}$, respectively, were attained at the pressure where the fcc structure first became stable. ${ }^{05}$ The $T_{c}$ was found to decrease with increasing pressure, but a secondary superconducting regime was observed in $\mathrm{YH}_{3}$ above $50 \mathrm{GPa}{ }^{95}$ Later work wherein the Eliashberg formalism was employed to estimate the $T_{c}$ yielded maximum values of $19.3 \mathrm{~K}$ for $\mathrm{ScH}_{3}$ at $18 \mathrm{GPa}$, and $22.5 \mathrm{~K}$ for $\mathrm{LaH}_{3}$ at $11 \mathrm{GPa} .{ }^{98}$ Another theoretical study found that the $T_{c}$ of $\mathrm{ScH}_{2}$ rises steeply under pressure reaching a maximum value of $38 \mathrm{~K}$ at $30 \mathrm{GPa}$, and then it plateaus near $31 \mathrm{~K}$ until at least $80 \mathrm{GPa} \cdot{ }^{[96}$

CSP studies have been employed to explore the phase diagram of $\mathrm{ScH}_{n}(n=1-3)$ up to $500 \mathrm{GPa} .{ }^{99}$ The monohydride was found to assume several phases that were calculated as being more stable at $1 \mathrm{~atm}$ than the previously suggested rock-salt structure, which became preferred 
at $10 \mathrm{GPa}$, followed by a transition to a $\mathrm{Cmcm}$ phase at $265 \mathrm{GPa}$. $\mathrm{ScH}_{2}$ was found to transform from a $\mathrm{CaF}_{2}$-type structure to one with $C 2 / m$ symmetry at $65 \mathrm{GPa}$. At $72 \mathrm{GPa}$, decomposition into $\mathrm{ScH}$ and $\mathrm{ScH}_{3}$ was computed to be enthalpically preferred, but higher pressures resulted in the stabilization of $\mathrm{ScH}_{2}$. $\mathrm{ScH}_{3}$ was computed to undergo the following set of transitions: $P 6_{3} \rightarrow$ $F m \overline{3} m \rightarrow P 6_{3} / m m c \rightarrow C m c m$ at 29,360 , and $483 \mathrm{GPa}$ respectively. Theoretical calculations have also been undertaken to predict the structure of $\mathrm{YH}_{3}$ at pressures where experimental data is not available. ${ }^{100101}$ Even though the estimated Debye temperature for a $\mathrm{Cmcm}$ phase at $225 \mathrm{GPa}$ was high, the DOS at $E_{F}$ suggested that the predicted phase would be superconducting only at low temperatures. ${ }^{100}$ Recently, $\mathrm{YH}_{3}$ compounds with the $P 2_{1} / \mathrm{m}$ and $I 4 / \mathrm{mmm}$ spacegroups were predicted to be stable, and their $T_{c}$ values were estimated as being $19 \mathrm{~K}$ and $9 \mathrm{~K}$ at $200 \mathrm{GPa}$, respectively. 101

A number of theoretical studies have recently appeared that investigated the higher hydrides of scandium with $n>3.102-105]$ Abe predicted the following stable phases: $I 4 / m m m-\mathrm{ScH}_{4}$ above $160 \mathrm{GPa}, P 6_{3} / m m c \mathrm{ScH}_{6}$ from $135-265 \mathrm{GPa}$, and above $265 \mathrm{GPa}$ an $I m \overline{3} m-\mathrm{ScH}_{6}$ structure isotypic with the $\mathrm{CaH}_{6}$ phase show in Fig. 2(a) that possesses $\left[4^{6} 6^{8}\right]$ polyhedra. ${ }^{102}$ The $\mathrm{ScH}_{4}$ structure contained $\mathrm{H}^{-}$as well as $\mathrm{H}_{2}^{\delta-}$ units as shown in Fig. 3.a), and it is isotypic with previously predicted phases for $\mathrm{CaH}_{4}{ }^{\frac{89}{}}$ and $\mathrm{SrH}_{4} \cdot{ }^{.091]}$ In addition to these structures the following phases have been predicted above $300 \mathrm{GPa}: \mathrm{Immm}-\mathrm{ScH}_{8}$, which was found by Qian et al., ${ }^{103}$ as well as $\mathrm{P}_{3} / \mathrm{mmc}_{\mathrm{ScH}}, \mathrm{Cmcm}-\mathrm{ScH}_{10}$, and $\mathrm{C} 2 / \mathrm{c}-\mathrm{ScH}_{12}$, which were found by Peng and co-workers. 104 Finally, Ye et al. showed that the $I 4_{1} m d-\mathrm{ScH}_{9}$ phase illustrated in Fig. 3(b) and $\mathrm{Immm}-\mathrm{ScH}_{12}$ had somewhat lower enthalpies than the previously proposed structures. ${ }^{105}$ In addition two new stable phases, $\mathrm{Cmcm}-\mathrm{ScH}_{6}$ and $\mathrm{Cmcm}-\mathrm{ScH}_{7}$, were predicted. It was therefore proposed that a large number of high hydrides of scandium could be synthesized above $150 \mathrm{GPa}$, and some of them were computed to have $T_{c}$ values as high as $\sim 200 \mathrm{~K} .{ }^{104} \mid 105$

Three manuscripts have explored superconductivity in higher hydrides of yttrium, $\mathrm{YH}_{n}, n \geq$ 4. 101106107 CSP techniques found that (in addition to $\mathrm{YH}_{3}$ ) $\mathrm{YH}_{4}$ and $\mathrm{YH}_{6}$ phases, which are isotypic to $I 4 / m m m-\mathrm{ScH}_{4}$ and $I m \overline{3} m-\mathrm{ScH}_{6}$, were thermodynamically and dynamically stable under pressure. At $120 \mathrm{GPa}$, the $T_{c}$ was estimated to be $84-95 \mathrm{~K}$ for the former and $251-264 \mathrm{~K}$ for the latter. ${ }^{106}$ Even though the $\mathrm{H}-\mathrm{H}$ distances at $120 \mathrm{GPa}$ in $\mathrm{Im} \overline{3} m-\mathrm{YH}_{6}$ were somewhat long, $1.31 \AA$, the ELF revealed covalent bonding interactions between the hydrogens. Another hydride of yttrium that was predicted to be superconducting at high temperatures was the sodalite-like $\mathrm{YH}_{10}$ phase illustrated in Fig. 3(c) whose $T_{c}$ was estimated as being 305-326 K at $250 \mathrm{GPa}$ via the Eliashberg equations. 107

Computational explorations of the hydrogen-rich phase diagram of lanthanum also led to the prediction of phases with the propensity for high temperature superconductivity. ${ }^{107}$ At $150 \mathrm{GPa}$ $\mathrm{LaH}_{n}$ with $n=2-5,8,10$ were found to be stable. $\mathrm{LaH}_{8}$ was comprised of an extended hydrogenic lattice and at $300 \mathrm{GPa}$ it's $T_{c}$ was estimated as being 114-131 K. $\mathrm{LaH}_{10}$ adopted a sodalitelike structure (isotypic to the $\mathrm{YH}_{10}$ phase show in Fig. 3.(c)) wherein the La atoms were arranged on an fcc lattice. This phase contained $\left[4^{6} 6^{12}\right]$ polyhedra, and numerically solving the Eliashberg equations yielded a $T_{c}$ of $257-274 \mathrm{~K}$ at $250 \mathrm{GPa}$ for $\mathrm{LaH}_{10}$. Its $T_{c}$ was found to decrease with increasing pressure. Remarkably, a superhydride of lanthanum consistent with the theoretically predicted structure for $\mathrm{LaH}_{10}$ was recently synthesized at $170 \mathrm{GPa} \cdot{ }^{108}$ Decompression led to a $F m \overline{3} m \rightarrow R \overline{3} m \rightarrow C 2 / m$ phase transformation.

Recently, an extensive theoretical investigation was carried out on the high hydrides of the 
(a)

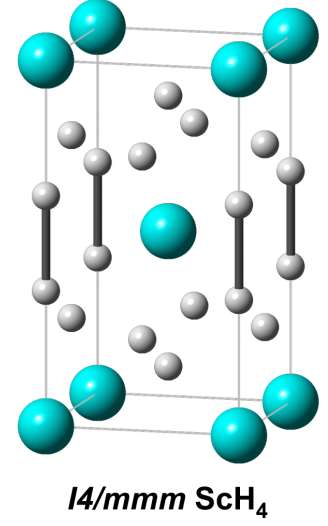

(b)

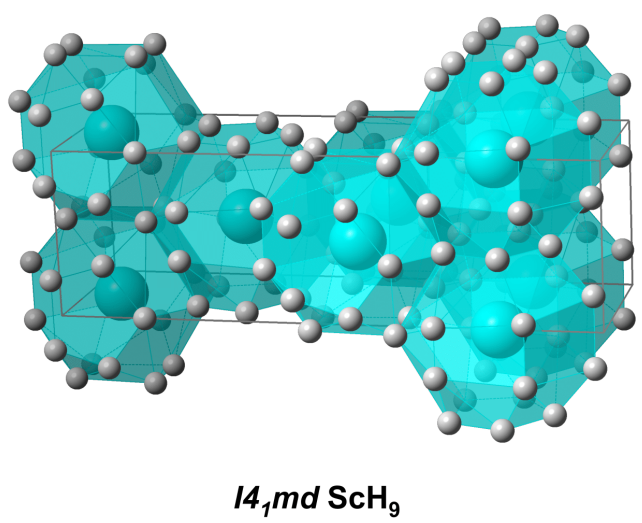

(c)

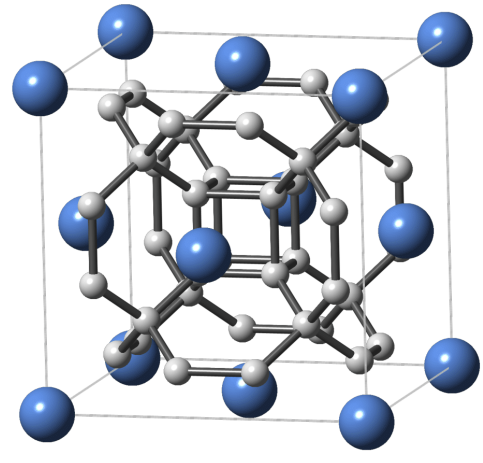

$F m \overline{3} m \mathrm{YH}_{10}$

Figure 3: Unit cell of (a) $I 4 / m m m-\mathrm{ScH}_{4} \frac{102-105}{\text { (which is isotypic with } \mathrm{YH}_{4} 101 / 106 / 107}$ and $\mathrm{LaH}_{4}{ }^{107}$ ), (b) $I 4_{1} m d-\mathrm{ScH}_{9},{ }^{105}$ and (c) $F m \overline{3} m-\mathrm{YH}_{10}$ 101106107 (which is isotypic with the recently predicted ${ }^{107}$ and synthesized ${ }^{108} \mathrm{LaH}_{10}$ phase).

REs under pressure. ${ }^{104}$ CSP calculations were performed to determine the most stable structures of the hydrides of Sc, Y, La, Ce and $\mathrm{Pr}$ and their convex hulls were obtained. It was assumed that the the polyhydrides of Nd, Pm, Sm, Eu, Gd, Tb, Dy, Ho, Er, Tm, Yb and Lu would be isotypic with phases that were found via CSP for the other REs. Therefore, the convex hulls for the hydrides of these twelve REs were generated by carrying out geometry optimizations on the predicted $\mathrm{REH}_{n}(n=3,4,6,9,10)$ species. The candidate phases considered were: $F m \overline{3} m$, $C m c m$, and $R \overline{3} m \mathrm{REH}_{3}, I 4 / m m m \mathrm{REH}_{4}, I m \overline{3} m, R \overline{3} c$, and $C 2 / m \mathrm{REH}_{6}, P 6_{3} / m m c, F \overline{4} 3 d$, and $P 6_{3} m \mathrm{REH}_{9}$, and $F m \overline{3} m, R \overline{3} m$, and $C m c m \mathrm{REH}_{10}$. The stable $\mathrm{REH}_{6}, \mathrm{REH}_{9}$ and $\mathrm{REH}_{10}$ phases resembled clathrate structures (examples of two of these, $I m \overline{3} m-\mathrm{MH}_{6}$ and $F m \overline{3} m-\mathrm{MH}_{10}$, are shown in Fig. 2(a) and Fig. 3(c), respectively) with $\mathrm{H}_{24}, \mathrm{H}_{29}$ and $\mathrm{H}_{32}$ cages surrounding the metal atoms, and $\mathrm{H}-\mathrm{H}$ distances of 1-1.2 $\AA$. The $T_{c}$ of some of these phases was estimated via solving the Eliashberg equations. $\mathrm{ScH}_{6}, \mathrm{ScH}_{9}, \mathrm{YH}_{6}, \mathrm{YH}_{9}, \mathrm{YH}_{10}$, and $\mathrm{LaH}_{6}$ had quite high $T_{c} \mathrm{~s}$, with predicted values of up to $303 \mathrm{~K}$ at $400 \mathrm{GPa}$ for $\mathrm{YH}_{10}$. The $T_{c}$ of $\mathrm{LaH}_{9}, \mathrm{CeH}_{9}, \mathrm{CeH}_{10}$ and $\mathrm{PrH}_{9}$ were significantly lower, $<56 \mathrm{~K}$, because they contained heavier elements.

\subsection{Group 4: Titanium, Zirconium, Hafnium}

The superconducting properties of $\mathrm{TiD}_{0.74}$ have been measured under pressure, and it was shown that $T_{c}$ varied from 4.17-4.43 K between $14-30 \mathrm{GPa},{ }^{109}$ somewhat lower than the value of $5.0 \mathrm{~K}$ obtained when a metastable form of this system was quenched to atmospheric pressures. As is common for many transition metal dihydrides, $\mathrm{TiH}_{2}$ crystallizes in the $\mathrm{CaF}_{2}$ (fcc) structure illustrated in Fig. 4(a) at room temperature. At lower temperatures a transition to the $14 / \mathrm{mmm}$ structure in Fig. 4(b) occurs. A recent DFT study computed a $T_{c}$ of $6.7 \mathrm{~K}$ and $2 \mathrm{mK}$ for the high and low temperature phases, respectively. ${ }^{110}$ Experiments revealed that the fcc $\rightarrow I 4 / \mathrm{mmm}$ transition also occurs at room temperature and $0.6 \mathrm{GPa}$, and suggested that this phase remains stable up to $90 \mathrm{GPa}{ }^{111} \mathrm{CSP}$ techniques, on the other hand, predicted the following sequence 
of transitions: $I 4 / \mathrm{mmm} \rightarrow P 4 / \mathrm{nmm} \rightarrow P 2_{1} / \mathrm{m}$ at 63 and $294 \mathrm{GPa}$, respectively. ${ }^{112}$ The computed XRD patterns of the $P 4 / \mathrm{nmm}$ structure were found to be in better agreement with experimental results up to $90 \mathrm{GPa}$ than those of $I 4 / \mathrm{mmm}$.

(a)

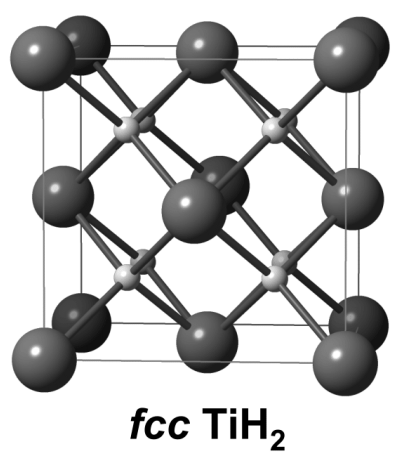

(b)

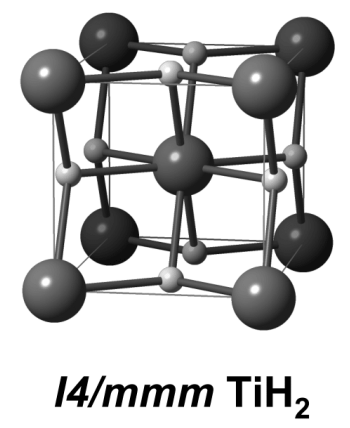

Figure 4: Two structures often adopted by metal hydrides: (a) the $\mathrm{CaF}_{2}$ (fcc) structure, and (b) the $I 4 / \mathrm{mmm}$ structure. For example, $\mathrm{TiH}_{2}$ assumes the fcc arrangement at ambient conditions, and the $I 4 / \mathrm{mmm}$ configuration at lower temperatures.

A number of phases have been reported in the $\mathrm{Zr} / \mathrm{H}$ phase diagram at ambient pressure, including two non-stoichiometric compounds: fcc $\delta$ - $\mathrm{ZrH}_{x}$ with $1.4<x<1.7$, and an $I 4 / \mathrm{mmm}$ symmetry $\epsilon-\mathrm{ZrH}_{x}$ structure, as well as a stoichiometric $P 4_{2} / n-\mathrm{ZrH}$ phase. ${ }^{113}$ At ambient conditions $\mathrm{ZrH}_{2}$ assumes the same $I 4 / \mathrm{mmm}$ symmetry structure as $\mathrm{TiH}_{2}$ (see Fig. 4(b)), and DFT calculations suggested that it would undergo an $I 4 / \mathrm{mmm} \rightarrow P 4 / \mathrm{nmm}$ transition at $103 \mathrm{GPa} .114$ Phases with $\mathrm{Zr}_{2} \mathrm{H}, \mathrm{ZrH}, \mathrm{ZrH}_{1.5}$, and $\mathrm{ZrH}_{2}$ stoichiometries have been studied theoretically, 115 and CSP techniques were employed to find stable phases with the stoichiometry $\mathrm{Zr}_{x} \mathrm{H}_{y}(x=$ $1, y=1-8 ; x=2, y=3,5)$ up to $150 \mathrm{GPa} \cdot{ }^{116}$ For pressures up to $100 \mathrm{GPa} \mathrm{ZrH}, \mathrm{ZrH}_{2}$ and $\mathrm{ZrH}_{3}$ were the only thermodynamically stable phases that were identified, whereas at $150 \mathrm{GPa}$ $\mathrm{ZrH}_{6}$ also emerged as a stable phase. The following structures were found to be dynamically stable and metallic at the pressures in the parentheses: $P 4_{2} / m m c-Z r H ~(0 \mathrm{GPa}), C m c m-\mathrm{ZrH}$ (120 GPa), $R \overline{3} m-\mathrm{ZrH}$ (150 GPa), I4/mmm-ZrH ${ }_{2}(50 \mathrm{GPa}), P m \overline{3} n-\mathrm{ZrH}_{3}(50 \mathrm{GPa})$, and $C m c 2_{1^{-}}$ $\mathrm{ZrH}_{6}(140 \mathrm{GPa})$. The only phase that exhibited a significant EPC, wherein the motions of the $\mathrm{Zr}$ atoms contributed towards $82 \%$ of the total $\lambda$, was $C m c m-\mathrm{ZrH}$, whose $T_{c}$ was estimated as being $10.6 \mathrm{~K}$ at $120 \mathrm{GPa}$.

The phases adopted by $\mathrm{HfH}_{2}$ under pressure, as well as their electronic structure and properties, were recently explored theoretically. $\frac{117}{17}$ The $14 / \mathrm{mmm}$ phase was calculated as being more stable than the $\mathrm{CaF}_{2}$ structure at atmospheric conditions (both structure types are shown in Fig. 4). A transition to a $C m m a$ structure at $180 \mathrm{GPa}$ was predicted, followed by a transformation to a $P 2_{1} / m$ phase at $250 \mathrm{GPa}$. The $T_{c}$ was estimated via the Allen-Dynes modified McMillan equation as being $47-193 \mathrm{mK}$ at $1 \mathrm{~atm}, 5.99-8.16 \mathrm{~K}$ at $180 \mathrm{GPa}$ and $10.62-12.8 \mathrm{~K}$ at $260 \mathrm{GPa}$ for the aforementioned phases. For each phase $T_{c}$ was found to decrease as the pressure increased.

At the time of writing this review the chemistry of the hydrides of titanium and of hafnium with unique stoichiometries had not yet been investigated. 


\subsection{Group 5: Vanadium, Niobium, Tantalum}

A number of molecular hydrides of the group 5 metals, including $\mathrm{VH}_{2}\left(\mathrm{H}_{2}\right), \mathrm{NbH}_{4}$ and $\mathrm{TaH}_{4}$ have been synthesized using laser ablation, and studied via molecular quantum mechanical calculations. 118

In the solid state experiments have shown that $\mathrm{VH}_{2}$ adopts the $\mathrm{CaF}_{2}$ structure, and CSP investigations have also found this to be the most stable phase at $1 \mathrm{~atm} .119$ A further transition to a Pnma phase was predicted at $50 \mathrm{GPa}$, and the $T_{c}$ was estimated as being $0.5 \mathrm{~K}$ and $4 \mathrm{~K}$ for $\mathrm{VH}_{2}$ at 0 and $60 \mathrm{GPa}$, respectively. This finding is in line with experiments that did not show any hints of superconductivity for $\mathrm{VH}_{n}, n<1.93$, above $1.5 \mathrm{~K} .120$ To the best of our knowledge, the structures and properties of vanadium hydrides with $n>2$ have not yet been explored computationally.

At ambient temperature and pressure phases with the stoichiometries $\mathrm{NbH}_{x}, x \leq 0.9$, are known, and various forms of $\mathrm{NbH}_{x}, x<1$, have been proposed to exist at different temperatures. $\mathrm{NbH}_{2}$ has been synthesized at 2 atm in the $\mathrm{CaF}_{2}$ structure shown in Fig. 4(a). DFT calculations found this to be the most stable phase at atmospheric pressures, and a transition to a $P 6_{3} m c$ phase was predicted to occur at $45 \mathrm{GPa} \cdot{ }^{119}$ The $T_{c}$ of $\mathrm{NbH}_{2}$ was estimated as being $1.5 \mathrm{~K}$ and $0.5 \mathrm{~K}$ at 0 and $60 \mathrm{GPa}$, respectively. A comprehensive theoretical investigation of $\mathrm{NbH}_{n}(n=0.75,1-6)$ up to $400 \mathrm{GPa}$ has been carried out. ${ }^{121}$ At 1 atm and $10 \mathrm{GPa} \mathrm{NbH}_{0.75}, \mathrm{NbH}$ and $\mathrm{NbH}_{2}$ were computed to be thermodynamically stable. At $50 \mathrm{GPa}$ the $\mathrm{NbH}_{3}$ stoichiometry joined them on the convex hull. By $400 \mathrm{GPa}$ species with $n=1-4$ were found to be stable. $C c c m-\mathrm{NbH}$ ( $1 \mathrm{~atm})$, $F m \overline{3} m-\mathrm{NbH}_{2}(50 \mathrm{GPa}), I \overline{4} 3 d-\mathrm{NbH}_{3}(100 \mathrm{GPa}), I 4 / m m m-\mathrm{NbH}_{4}(300 \mathrm{GPa})$ and $C m m m-\mathrm{NbH}_{6}$ $(400 \mathrm{GPa})$ were good metals at the pressures given in the parentheses, suggesting that all of them have the potential to be superconductors. Due to the computational expense involved the $T_{c}$ of only a few phases could be estimated via the Allen-Dynes modified McMillan equation. The $T_{c}$ values were calculated to be: $1.5-2.4 \mathrm{~K}$ for $\mathrm{NbH}$ at $1 \mathrm{~atm}, 1.5-2.6 \mathrm{~K}$ and $0.7-1.5 \mathrm{~K}$ for $\mathrm{NbH}_{2}$ at 1 atm and $50 \mathrm{GPa}$, respectively, and 38-47 K for $\mathrm{NbH}_{4}$ at $300 \mathrm{GPa}$. The reason for the higher $T_{c}$ obtained for $\mathrm{NbH}_{4}$ can be traced back to the larger $\lambda$ and average logarithmic frequency $\left(\omega_{\log }\right)$, which is a result of the presence of a larger mole ratio of hydrogen as compared to the other phases. Within the framework of strongly-coupled Eliashberg theory, the $T_{c}$ of $\mathrm{NbH}_{4}$ was calculated as being somewhat higher, $49.6 \mathrm{~K} .{ }^{122} \mathrm{~A}$ recent combined experimental and theoretical study showed that $\mathrm{NbH}_{2.5}$ could be synthesized below $46 \mathrm{GPa}$, and above $56 \mathrm{GPa}$ the $\mathrm{NbH}_{3}$ phase illustrated in Fig. 5(a) was made. ${ }^{123}$ The formation of some of the phases that were experimentally observed could only be explained when finite temperature contributions to the free energy were considered.

A number of $\mathrm{Ta}_{x} \mathrm{H}_{y}$ compounds with varying stoichiometries including $\mathrm{Ta}_{2} \mathrm{H}$ and $\mathrm{TaH}_{2}$ have been synthesized at ambient conditions. A review of the experimental studies, coupled with the results of DFT calculations that investigated the structures and electronic structures of the hydrides of tantalum up to $300 \mathrm{GPa}$ is given in Ref. ${ }^{[124}$ This study showed that the XRD patterns for the theoretically predicted ambient pressure phases of $\mathrm{Ta}_{2} \mathrm{H}(C 222)$ and $\mathrm{Ta}_{5} \mathrm{H}(C 2)$, shown in Fig. 5(b,c), agreed well with those determined experimentally. The computed enthalpy of a $\mathrm{P}_{3} m c$ symmetry $\mathrm{TaH}_{2}$ phase was somewhat lower than that of the experimentally found $F m \overline{3} m$ $\left(\mathrm{CaF}_{2}\right.$ type, see Fig. $\left.4 \mathrm{~b}\right)$ structure. At 1 atm only $\mathrm{TaH}$ and $\mathrm{TaH}_{2}$ were thermodynamically stable. By $50 \mathrm{GPa} \mathrm{TaH}_{3}$ and $\mathrm{TaH}_{4}$ joined them on the convex hull, and $\mathrm{TaH}_{6}$ became stable by $270 \mathrm{GPa}$. 
(a)

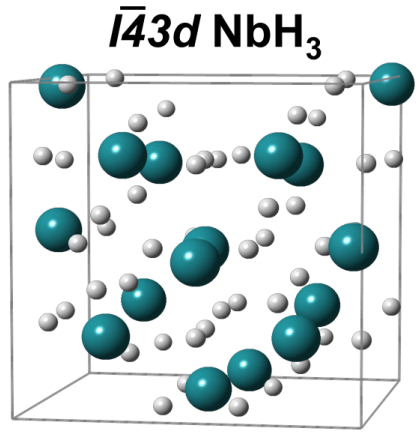

(b)

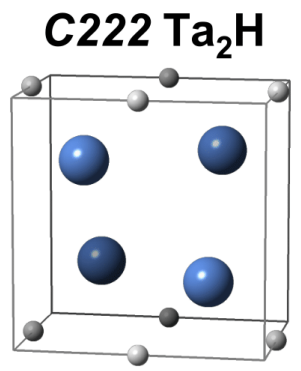

(c)

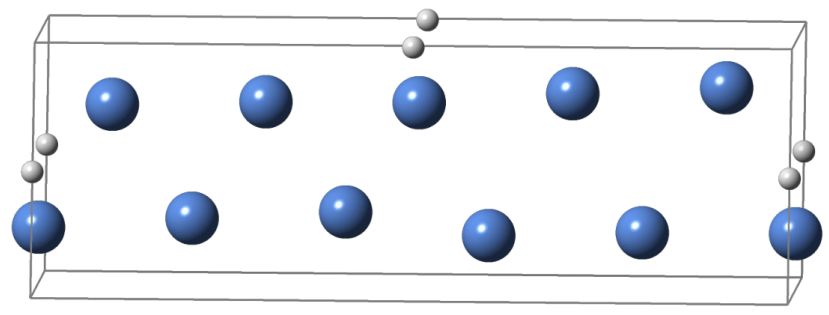

$\mathrm{C2} \mathrm{Ta}_{5} \mathrm{H}$

Figure 5: Hydrides of group 5 transition metals that have been synthesized and studied theoreti-

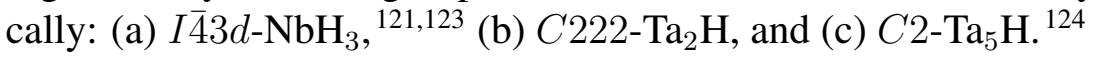

Molecular $\mathrm{H}_{2}$ units were absent in the most stable structures, which were all metallic. Phonon calculations verified dynamic stability for $P n m a-\mathrm{TaH}_{2}, R \overline{3} m-\mathrm{TaH}_{4}$ and $F d d 2-\mathrm{TaH}_{6}$ at 200, 250 and $300 \mathrm{GPa}$, respectively. At these pressures the $T_{c}$ s were predicted to be 5.4-7.1 K, 23.9-31 K, and 124.2-135.8 K, respectively. Recently, a new dihydride of tantalum that had an hcp metal lattice was synthesized under pressure. 125

\subsection{Group 6: Chromium, Molybdenum, Tungsten}

In the solid state at $1 \mathrm{~atm} \mathrm{CrH}, \mathrm{MoH}$ and $\mathrm{WH}$ crystallize in an anti-NiAs structure, shown in Fig. 6(a), wherein the hydrogen atoms are found in the interstitial sites. Less is known about a cubic polymorph of $\mathrm{CrH}$, because of the difficulties of preparing it reproducibly. ${ }^{[26] 127}$ The dihydride and trihydride of chromium have been made, but they have not yet been structurally characterized. Molecular hydrides of the group 6 transition metals have been synthesized with a wide range of stoichiometries, including species with high hydrogen content such as $\left(\mathrm{H}_{2}\right)_{2} \mathrm{CrH}_{2},{ }^{128} \mathrm{MoH}_{6},{ }^{129}$ $\mathrm{WH}_{6}, \frac{130}{}$ and $\mathrm{WH}_{4}\left(\mathrm{H}_{2}\right)_{4} \cdot \frac{131}{}$ In fact, quantum chemical calculations showed that the formation of $\mathrm{MH}_{12}(\mathrm{M}=\mathrm{Cr}, \mathrm{Mo}, \mathrm{W})$ from $\mathrm{MH}_{6}$ and $3 \mathrm{H}_{2}$ molecules is energetically favorable. ${ }^{132}$ The existence of these molecules has led to the speculation that high pressure could potentially be used to stabilize high hydrides of the group 6 transition metals, despite the fact that such species are not known at ambient conditions.

Recently, CSP has been employed to predict the most stable structures of compounds with 
the $\mathrm{Cr}_{x} \mathrm{H}_{y}$ stoichiometry up to pressures of $300 \mathrm{GPa} \cdot \frac{133}{\sqrt{13}}$ Whereas at $1 \mathrm{~atm} \mathrm{CrH}$ was the only thermodynamically stable phase found, pressure promoted the stabilization of hydrogen rich phases. When ZPE effects were included $\mathrm{Cr}_{2} \mathrm{H}_{3}, \mathrm{CrH}_{2}, \mathrm{CrH}_{3}, \mathrm{CrH}_{4}$, and $\mathrm{CrH}_{8}$ were found to lie on the convex hull at some pressure. All of these structures contained the common feature that the metal sublattices were hexagonal close-packed, and hydrogen atoms were found in the octahedral or tetrahedral sites. The EPC parameter was calculated for $\mathrm{CrH}$ and $\mathrm{CrH}_{3}$ as representative structures for these phases, and the $T_{c}$ was estimated using the Allen-Dynes equation. The $T_{c}$ of $\mathrm{CrH}$ was calculated as being $10.6 \mathrm{~K}$ at $0 \mathrm{GPa}$, and it was found to decrease with increasing pressure to $3.1 \mathrm{~K}$ at $200 \mathrm{GPa}$. Because of the increased hydrogen content, the $\mathrm{EPC}$ and $\omega_{\text {log }}$ of $\mathrm{CrH}_{3}$ was calculated as being larger than that of $\mathrm{CrH}$, with a concomitantly larger $T_{c}$ of $37.1 \mathrm{~K}$ at $81 \mathrm{GPa}$. Again, $T_{c}$ decreased under pressure, dropping to $\sim 28 \mathrm{~K}$ at $200 \mathrm{GPa}$.

Recent experiments uncovered the crystal structures assumed when Mo was subject to hydrogen pressures up to $30 \mathrm{GPa} \cdot \frac{134}{}$ At $4 \mathrm{GPa}$ a phase transformation from a bcc to an hep metal hydride occurred. The H:Mo ratio increased continuously with pressure and reached a saturation limit of 1.35:1 at about $15 \mathrm{GPa}$. First principles calculations have shown that phases with $\mathrm{MoH}$ and $\mathrm{MoH}_{2}$ stoichiometries are dynamically stable from $0-100 \mathrm{GPa} \cdot{ }^{135]}$ Whereas $P 6_{3} / \mathrm{mmc}-\mathrm{MoH}$ was found on both the $20 \mathrm{GPa}$ and $100 \mathrm{GPa}$ convex hulls, $\mathrm{MoH}_{2}$ only became thermodynamically stable by $100 \mathrm{GPa}$, and $\mathrm{MoH}_{3}$ did not lie on the convex hull between 0-100 GPa. At $2 \mathrm{GPa}$ the most stable $\mathrm{MoH}_{2}$ phase assumed the $P 6_{3} m c$ spacegroup, and a Pnma phase became preferred past $24 \mathrm{GPa}$. In all of the stable structures Mo atoms were found in the hexagonal sites and $\mathrm{H}$ atoms in the octahedral and tetrahedral sites. Even though both $\mathrm{MoH}$ and $\mathrm{MoH}_{2}$ were metallic at $100 \mathrm{GPa}$, the DOS at $E_{F}$ was dominated by metal $d$-states, suggesting that their $T_{c} \mathrm{~s}$ will be lower than that of elemental Mo.

(a)

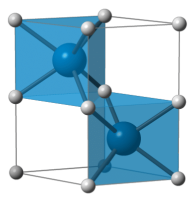

$P 6_{3} / m m c$ WH

(c)

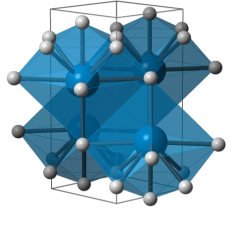

$\mathrm{P4} / \mathrm{nmm} \mathrm{WH}_{4}$ (b)

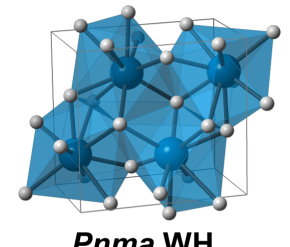

(d)

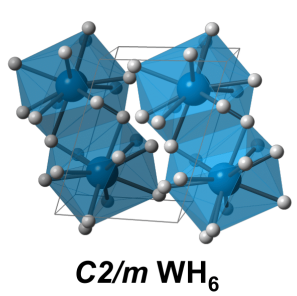

Figure 6: The hydrides of tungsten studied theoretically and experimentally in Ref. $\frac{136}{13 H}$ has been synthesized in the anti-NiAs $\left(P 6_{3} / m m c\right.$ symmetry) structure under pressure. 1361137

Because the solubility of hydrogen in tungsten is low, this metal is often employed as a gasket material to seal hydrogen in DACs at high pressure. However, during an experimental study of a mixture of $\mathrm{H}_{2}$ and $\mathrm{SiH}_{4}$ under pressure, diffraction measurements showed evidence for the serendipitous formation of a tungsten hydride. $\frac{138}{1}$ This, along with the synthesis of a number 
of molecular hydrides of tungsten in low temperature matrices (see Ref. $\frac{131}{1}$ and Refs. within) inspired the computational search for high hydrides of tungsten in the solid state at 1 atm and under pressure. ${ }^{136 / 139}$ Some of the phases predicted are illustrated in Fig. 6. CSP studies found $\mathrm{WH}$ and $\mathrm{WH}_{4}$ phases that lay on the convex hull at $25 \mathrm{GPa}$, where they were joined by $\mathrm{WH}_{2}$ by $100 \mathrm{GPa}$, and $\mathrm{WH}_{6}$ by $150 \mathrm{GPa} \cdot{ }^{136}$ Moreover, computational experiments hinted that $\mathrm{WH}_{6}$ has the ability to polymerize at ambient conditions. $\stackrel{139}{ }$ Phonon calculations confirmed that all of these phases were dynamically stable at $150 \mathrm{GPa}$. Only $\mathrm{WH}$ and $\mathrm{WH}_{2}$ were found to be good metals, whereas the Fermi level of $\mathrm{WH}_{4}$ and $\mathrm{WH}_{6}$ lay in a pseudogap. Unfortunately, attempts to synthesize hydrides beyond $\mathrm{WH}$, which was found to crystallize in an anti-NiAs structure, were not successful. A more recent computational and experimental study confirmed the synthesis of WH by $25 \mathrm{GPa} \cdot{ }^{137}$ However, it was shown that between 25 and $50 \mathrm{GPa}$ the equation of states data was best explained by a combination of $\mathrm{WH}$ and $\mathrm{WH}_{2}$, suggesting that the maximum $\mathrm{H}: \mathrm{W}$ ratio attained in the synthesis is $\sim 1 \frac{1}{3}$. The superconducting properties of the compressed tungsten hydride phases has so far not been explored theoretically or experimentally.

\subsection{Group 7: Manganese, Technetium, Rhenium}

At the time of writing this manuscript, very little had been reported about the high pressure structures of the group 7 hydrides, and their propensity for superconductivity. On the other hand, molecular hydrides of these elements such as $\mathrm{MnH}_{2}$ and $\mathrm{ReH}_{4}$ have been made via laser ablation, and their electronic structure has been studied via first-principles calculations. 140

$\mathrm{MnH}_{x}$ phases with $x<1$ are known to adopt structures that are based on fcc, hcp and double hexagonal closed packed (dhcp) metal lattices, and their phase diagrams have been studied as a function of $\mathrm{H} / \mathrm{Mn}$ content up to $1000{ }^{\circ} \mathrm{C}$ and $7.6 \mathrm{GPa} . \frac{141 / 142}{}$

Below $2 \mathrm{GPa}$ the $\mathrm{H}$ :Tc ratio in hydrides of technetium is less than 1 , and the $T_{c}$ of the hydrogenated system is lower than that of the pure metal. ${ }^{143 \mid 144}$ Theoretical CSP investigations found that at $50 \mathrm{GPa} P 6_{3} / m m c-\mathrm{TcH}$ is the only thermodynamically stable phase, but by $150 \mathrm{GPa}$ $I 4 / m m m-\mathrm{TcH}_{2}$ and $\mathrm{Pnma}-\mathrm{TcH}_{3}$ also lie on the convex hull. ${ }^{145} \mathrm{By} 200 \mathrm{GPa} \mathrm{TcH}_{2}$ is no longer thermodynamically stable. However, these phases, and others, are metastable across a broad pressure range. The Allen-Dynes modified McMillan equation was employed to estimate the following $T_{c} \mathrm{~s}$ : $5.4-10.7 \mathrm{~K}$ for $I 4 / m m m-\mathrm{TcH}_{2}$ between $100-200 \mathrm{GPa}, 8.6 \mathrm{~K}$ for $C m c m-\mathrm{TcH}_{2}$ at $300 \mathrm{GPa}$, and $9.9 \mathrm{~K}$ for $\mathrm{P}_{2} / m m c-\mathrm{TcH}_{3}$ at $300 \mathrm{GPa}$.

An experimental study showed that above $50 \mathrm{GPa}$ silane decomposes and the released $\mathrm{H}_{2}$ reacts with metals in the DAC to form metal hydrides. ${ }^{146}$ At $50 \mathrm{GPa}$ the diffraction pattern of the rhenium metal indicated that its volume had expanded, presumably due to the uptake of hydrogen. Based upon the volume it was proposed that a compound with $\mathrm{ReH}_{0.39}$ stoichiometry had formed, in agreement with previous studies, $\frac{147}{}$ and this species was found to be stable up to at least $108 \mathrm{GPa}$. An $\mathrm{ReH}_{0.5}$ stoichiometry was synthesized at $15 \mathrm{GPa}$ in the layered anti-CdI $\mathrm{C}_{2}$ structure, and heating this compound promoted a phase transition to the NiAs structure type wherein the hydrogen content was increased to $\mathrm{ReH}_{0.85}$. 148 


\subsection{Group 8: Iron, Ruthenium, Osmium}

The Earth's core is composed primarily of iron alloyed with nickel and light elements. However, because seismic models suggest that the density of the Earth's core is several percent lower than estimates made for iron-nickel alloys, it has been proposed that iron hydrides may be important constituents of the core. A number of experiments have shown that pressure dramatically increases the solubility of hydrogen in iron $\frac{149}{y i e l d i n g ~} \mathrm{Fe}: \mathrm{H}$ ratios approaching $1: 1 \stackrel{150}{1}$ that assume a number of potential structure types including dhcp, hcp and fcc. $151-153$ DFT calculations on FeH up to $130 \mathrm{GPa}$ suggested the following sequence of structural phase transitions: $\mathrm{dhcp} \rightarrow \mathrm{hcp} \rightarrow$ fcc. 154

The propensity for the stabilization of novel stoichiometries under pressure has inspired theoretical and experimental exploration of iron hydrides with non-classical compositions. Evolutionary structure searches coupled with DFT calculations were employed to predict the most stable $\mathrm{Fe}_{x} \mathrm{H}_{y}(x=1-4, y=1-4)$ structures at pressures of 100-400 GPa. ${ }^{155}$ Even though $\mathrm{FeH}$ was the lowest point on the convex hull within the whole pressure range studied, all of the other stoichiometries either lay on the hull or close to it. For $\mathrm{Fe}_{x} \mathrm{H}$ with $x \geq 1$ the iron atoms in the most stable structures assumed close-packed lattices, and the hydrogen atoms were located in the octahedral voids. At pressures similar to those in the center of the Earth, FeH was found to adopt a rock-salt structure wherein the iron atoms were fcc-packed. At 300 and $400 \mathrm{GPa}$ the preferred $\mathrm{FeH}_{3}$ geometries were predicted to assume the $\mathrm{Cu}_{3} \mathrm{Au}$ structure (spacegroup $\mathrm{Pm} \overline{3} m$ ), and the $\mathrm{Cr}_{3} \mathrm{Si}(P m \overline{3} n)$ structure types, respectively. At both of these pressures the most stable $\mathrm{FeH}_{4}$ structure adopted $P 2_{1} / m$ symmetry. A theoretical investigation focused on the $\mathrm{FeH}_{4}$ stoichiometry from $80-400 \mathrm{GPa} \cdot{ }^{156} \mathrm{CSP}$ suggested that the following pressure induced phase transitions would occur in this phase: $P 2_{1} 3 \rightarrow I m m a \rightarrow P 2_{1} / m$ at 109 and $242 \mathrm{GPa}$, respectively. Only $\mathrm{Imma}_{\mathrm{meH}} \mathrm{FeH}_{4}$ was found to be a metal with the HSE hybrid functional. Its $T_{c}$ was estimated as being $1.7 \mathrm{~K}$ using the Allen-Dynes modified McMillan equation, where $75 \%$ of the EPC originated from the motions of the hydrogen atoms. Recent evolutionary structure searches have predicted hitherto unknown phases to be stable above $150 \mathrm{GPa}: P 4 / m m m-\mathrm{Fe}_{3} \mathrm{H}_{5}, I m m m-\mathrm{Fe}_{3} \mathrm{H}_{13}$, $I 4 / \mathrm{mmm}^{-\mathrm{FeH}_{5}}$, and $C m m m-\mathrm{FeH}_{6}$ whose $T_{c}$ was estimated to be $43 \mathrm{~K}$ at $150 \mathrm{GPa}$. 157

The work of Bazhanova et al. ${ }^{[155}$ inspired a combined experimental/theoretical investigation

wherein laser heating of a DAC was employed to synthesize higher hydrides of iron. ${ }^{158}$ In the experiment the dhcp-FeH structure illustrated in Fig. 7(a), which was calculated to undergo a ferromagnetic $(\mathrm{FM})$ to nonmagnetic $(\mathrm{NM})$ transition at $45 \mathrm{GPa}$, was observed. At $67 \mathrm{GPa}$ hydrogen uptake occurred leading to an $I 4 / m m m-\mathrm{FeH}_{\sim 2}$ phase with $\mathrm{FM}$ order. A recent study showed that the positions of the hydrogen atoms in the most stable dihydride phase, illustrated in Fig. 7 (b), differed from those proposed in the original study. ${ }^{[57}$ The NM $P m \overline{3} m-\mathrm{FeH}_{3}$ phase shown in Fig. 7(c), which was previously predicted by CSP in Ref., ${ }^{155}$ formed at $86 \mathrm{GPa}$. Calculations showed that all of the synthesized phases were metallic, hinting that they could potentially be superconducting. The FM to NM transition of the hcp and dhcp phases of FeH under pressure have been studied via DFT calculations. 159

Recently, the $\mathrm{FeH}_{5}$ phase illustrated in Fig. 7(d) was synthesized after laser heating in a DAC above $130 \mathrm{GPa} .{ }^{160}$ This phase contained $\mathrm{FeH}_{3}$ units separated by slabs of atomic hydrogen wherein the $\mathrm{H}-\mathrm{H}$ distances resembled those that would be found in bulk atomic hydrogen. Calculations predicted this phase to be superconducting below $\sim 50 \mathrm{~K}$ around $150 \mathrm{GPa} .157 / 161$ 


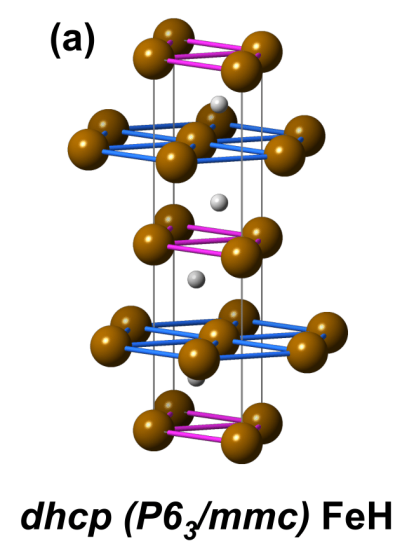

(b)

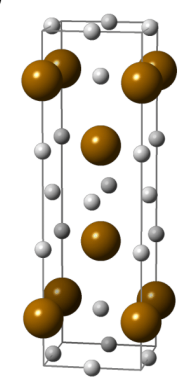

14/mmm $\mathrm{FeH}_{2}$ (c)

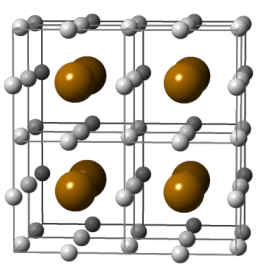

$P m \overline{3} m \mathrm{FeH}_{3}$ (d)

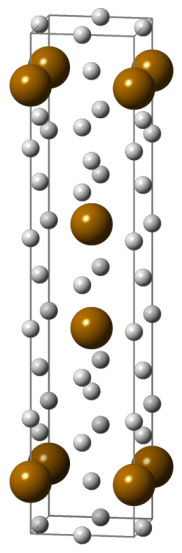

I4/mmm $\mathrm{FeH}_{5}$

Figure 7: Iron hydride phases that have been synthesized under pressure (a) dhcp-FeH, 158 (b) $I 4 / m m m-\mathrm{FeH}_{2},{ }^{158}$ (c) $\mathrm{Pm} \overline{3} m-\mathrm{FeH}_{3}, \stackrel{158}{{ }^{15}}$ and (d) $I 4 / m m m-\mathrm{FeH}_{5} .160$

Experiments carried out to $9 \mathrm{GPa}$ have not provided evidence for the formation of hydrides of ruthenium. However, CSP studies suggested that a monohydride in the fcc structure (also known as the $\mathrm{NaCl}$ structure with $F m \overline{3} m$ symmetry) would become stable above $10 \mathrm{GPa} .{ }^{162]} \mathrm{In}$ a later study of $\mathrm{RuH}_{n}(n=1-8)$ from 50-300 GPa three stoichiometries were found to lie on the convex hull. ${ }^{163} \mathrm{Fm} \overline{3} \mathrm{~m}$ - $\mathrm{RuH}$ ( $\mathrm{NaCl}$ type) became stable above $7 \mathrm{GPa}$ and remained the preferred monohydride until $300 \mathrm{GPa}$. $\mathrm{RuH}_{3}$ emerged as a stable structure at $66 \mathrm{GPa}$, assuming the $P m \overline{3} m$ structure illustrated in Fig. 7( c) below $120 \mathrm{GPa}$, and a $P m \overline{3} n$ symmetry structure at higher pressures. Another stable phase, $\mathrm{RuH}_{6}$, adopted the $\mathrm{Pm}$ spacegroup at $19.5 \mathrm{GPa}$ and transitioned to an Imma symmetry phase at $95 \mathrm{GPa}$. By $100 \mathrm{GPa}$, however, the decomposition of $\mathrm{RuH}_{6}$ to $\mathrm{RuH}_{3}$ and $\mathrm{H}_{2}$ was found to be thermodynamically preferred. Whereas the mono and trihydride only contained atomistic hydrogen, $\mathrm{RuH}_{6}$ was also comprised of molecular $\mathrm{H}_{2}$ units. $\mathrm{RuH}$ and $\mathrm{RuH}_{3}$ were found to be metallic, whereas $\mathrm{RuH}_{6}$ was semi-conducting. The $T_{c}$ for $\mathrm{RuH}$ was estimated as being $0.41 \mathrm{~K}$ at $100 \mathrm{GPa}$, and for $\mathrm{RuH}_{3}$ it was found to be $3.57 \mathrm{~K}$ and $1.25 \mathrm{~K}$ at $100 \mathrm{GPa}$ and $200 \mathrm{GPa}$, respectively. A subsequent experimental study illustrated that a hydride of ruthenium could be synthesized in a DAC between 14-30 GPa. ${ }^{164}$ The phase was identified as the monohydride wherein the metal lattice had the fcc structure and the hydrogen atoms were located on the octahedral sites, in agreement with the original prediction by Gao et al. 162

Evolutionary structure searches were carried out to identify new phases of $\mathrm{OsH}_{n}(n=1-8)$ from 50-300 GPa. ${ }^{165}$ Three stable stoichiometries were predicted, namely, OsH ( $P \geq 94 \mathrm{GPa}$ ), $\mathrm{OsH}_{3}(P=140-246 \mathrm{GPa})$, and $\mathrm{OsH}_{6}(P=38-155 \mathrm{GPa})$. OsH and $\mathrm{OsH}_{3}$ adopted a single stable phase (with $F m \overline{3} m$ and $C m m 2$ symmetry, respectively), while $\mathrm{OsH}_{6}$ assumed a $P 2_{1} / c$ phase below, and an $F d d 2$ phase above $104 \mathrm{GPa}$. Whereas $\mathrm{OsH}$ and $\mathrm{OsH}_{3}$ were found to be good metals, $\mathrm{OsH}_{6}$ was semi-conducting. The hydrogenic sublattices of the stable and metastable phases contained some of the following structural motifs: $\mathrm{H}, \mathrm{H}_{2}$, as well as linear, bent and triangular $\mathrm{H}_{3}$ units. The estimated $T_{c}$ of $\mathrm{OsH}$ was $2.1 \mathrm{~K}$ at $100 \mathrm{GPa}$, which is higher than that of pure osmium metal. 


\subsection{Group 9: Cobalt, Rhodium, Iridium}

Recent experiments carried out in a DAC show that cobalt undergoes a two-step hydrogenation process at and above room temperature. $\frac{166}{}$ Above $2.7 \mathrm{GPa}$ the hcp structure was maintained and hydrogen uptake resulted in a $\mathrm{CoH}_{0.6}$ stoichiometry, followed by a transformation to a fcc $\mathrm{CoH}_{0.9}$ structure above $4.2 \mathrm{GPa} . \frac{166}{}$ Between $5-11 \mathrm{GPa}$ the monohydride, FM fcc-CoH, was formed. Recent experiments carried out in a DAC up to $22 \mathrm{GPa}$ led to hydrogen uptake by cobalt at $\sim 4 \mathrm{GPa} .{ }^{167}$ Above this pressure a monohydride with the fcc structure formed, and the hydrogen content could not be increased further. Neutron diffraction experiments have shown that hydrogen occupies octahedral sites in both the hcp ${ }^{168}$ and fcc ${ }^{169}$ structures. Despite the fact that higher hydrides of cobalt have not been synthesized in the solid state, the molecular hydrides $\mathrm{CoH}, \mathrm{CoH}_{2}$, and $\mathrm{CoH}_{3}$ have been created in an electric field, $\stackrel{170}{ }$ and studied via unrestricted calculations using the B3LYP functional. ${ }^{171}$ Moreover, CSP studies have found that $F m \overline{3} m-\mathrm{CoH}_{2}$, $I 4 / m m m-\mathrm{CoH}_{2}$, and $\mathrm{Pm} \overline{3} m-\mathrm{CoH}_{3}$ (the latter two structures are isotypic with the $\mathrm{FeH}_{2}$ and $\mathrm{FeH}_{3}$ structures shown in Fig. 7(b,c)) are stable between 10-42 GPa, 42-300 GPa, and 30-300 GPa, respectively. ${ }^{172}$ Even though these ionic solids were metallic, calculations did not reveal superconductivity up to $200 \mathrm{GPa}$.

Rhodium assumes the fcc structure, with one octahedral and two tetrahedral sites per metal atom. In the monohydride, hydrogen atoms occupy the octahedral sites. Calculations have shown that $\mathrm{RhH}$ in the $\mathrm{NaCl}$ structure type has a magnetic moment of $0.45 \mu_{B}$ at $1 \mathrm{~atm}, \frac{173}{12}$ and predicted that it would undergo the following structural phase transitions: $\mathrm{NaCl} \rightarrow$ zincblende $\rightarrow \mathrm{CsCl} \rightarrow \mathrm{NiAs}$, at 11,154 and $382 \mathrm{GPa} \cdot{ }^{174}$ First principles calculations suggested that under mild pressures an $\mathrm{RhH}_{2}$ stoichiometry, which is isotypic with the $\mathrm{TiH}_{2}$ structure shown in Fig. 4. wherein each tetrahedral site was filled with a hydrogen atom, and each octahedral site was vacant, would be the thermodynamically preferred hydride of rhodium. ${ }^{175}$ In this same study experiments were carried out in a DAC up to $19 \mathrm{GPa}$ revealing $\mathrm{RhH}$ formation above $4 \mathrm{GPa}$ (in agreement with previous experiments $\frac{176}{}$ ), and $\mathrm{RhH}_{2}$ formation above $8 \mathrm{GPa}$. Upon decompression dehydrogenation occurred, yielding the pure metal by $3 \mathrm{GPa}$ at room temperature. At low temperatures the dihydride could be quenched to atmospheric pressures. This was the first dihydride of the platinum group metals to be synthesized.

Until 2013, no binary hydride of iridium was known. Experiments up to $125 \mathrm{GPa}$ wherein the metal was compressed in a DAC in a hydrogen medium revealed the formation of a new phase at $55 \mathrm{GPa} \cdot{ }^{177}$ The difference in volume between this phase and that of the pure metal suggested that a hydride with the $\mathrm{IrH}_{3}$ stoichiometry had formed, and XRD patterns showed the metal lattice assumed the same $P m \overline{3} m$ structure as $\mathrm{FeH}_{3}$ (see Fig. 7(c)). On decompression, decomposition of the trihydride began at 15-20 GPa, and it was not fully complete by $6 \mathrm{GPa}$. First-principles calculations verified that the synthesized phase is most likely a trihydride, and the thermodynamic and dynamic stability, as a function of pressure of various structural candidates was explored. The experiments did not support the existence of a Pnma structure that was found to have the lowest enthalpy above $68 \mathrm{GPa}$, and it was hypothesized that the formation of this phase was hindered by a kinetic barrier. At the same time Zaleski-Ejgierd carried out an independent theoretical study of the hydrides of iridium under pressure. ${ }^{178}$ Various CSP techniques were used to predict the most stable structures for a wide range of hydrogen content at 25 and $125 \mathrm{GPa}$. At $50 \mathrm{GPa}$ a dynamically stable $\mathrm{P}_{3}{ }_{3} \mathrm{mc}-\mathrm{IrH}_{3}$ phase, which was semiconducting at $25 \mathrm{GPa}$, was the lowest 
point on the convex hull. At low pressures this phase was comprised of molecular $\mathrm{IrH}_{3}$ units. A metallic $\mathrm{IrH}_{2}$ phase had the most negative $\Delta H_{F}$ at 100-125 GPa. The experimentally observed $P m \overline{3} m-\mathrm{IrH}_{3}$ phase was also found via CSP, but its enthalpy was higher than that of $\mathrm{P}_{3} m c-\mathrm{IrH}_{3}$ throughout the pressure range studied. Interestingly, the $\mathrm{Pnma}-\mathrm{IrH}_{3}$ phase predicted by Scheler and co-workers ${ }^{177}$ had nearly the same structure as $P 6_{3} m c-\mathrm{IrH}_{3}$ found by Zaleski-Ejgierd.

\subsection{Group 10: Nickel, Palladium, Platinum}

Hydrogenation of FM Ni to $\mathrm{NiH}_{x}$ occurs at $\sim 0.8 \mathrm{GPa}$, and an fcc structure is formed. ${ }^{166}$ As $x$ increases the magnetization drops, with the formation of the paramagnetic (PM) phase occurring by $x \sim 0.6 .179$ The disappearance of the magnetic state upon hydrogenation has been observed in Linear Muffin Tin Orbital (LMTO), ${ }^{180}$ full-potential linear augmented plane-wave (FLAPW), 166 and pseudopotential plane-wave calculations. $\frac{181}{1}$ The latter suggest that the most stable $\mathrm{NiH}_{x}$ structures up to $210 \mathrm{GPa}$ have a metal fcc lattice with the hydrogen atoms filling the octahedral sites. ${ }^{181}$ Moreover, the concentration at which the FM to PM transition is calculated to occur decreases under pressure from $\mathrm{NiH}_{0.375}$ at $210 \mathrm{GPa}$ to $\mathrm{NiH}_{0.6875}$ at $4 \mathrm{GPa}$.

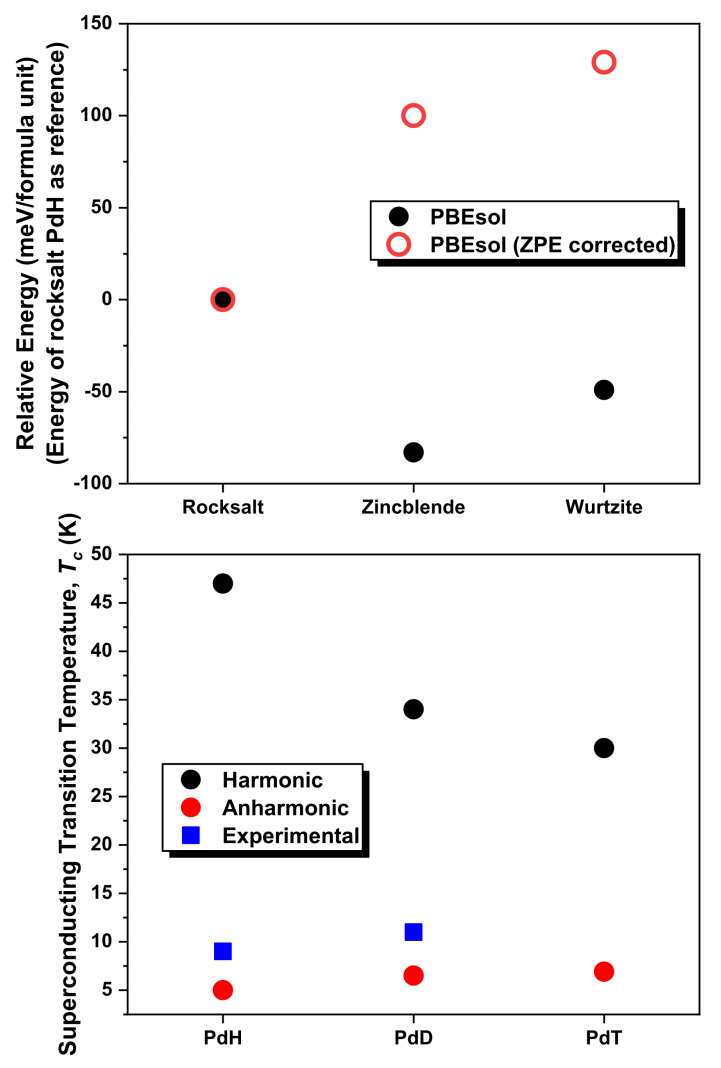

Figure 8: (top) The relative energies of different $\mathrm{PdH}$ structures at 1 atm with (open circles) and without (closed circles) the ZPE corrections. ${ }^{182}$ (bottom) The experimental superconducting temperature of $\mathrm{PdH}$ and its isotopes at $1 \mathrm{~atm}, \frac{183}{2}$ along with theoretically computed values. $\frac{184}{1}$

Palladium hydride, one of the first transition metal hydrides to be synthesized, was first made 
over 150 years ago. 185 At atmospheric pressure the metal lattice adopts an fcc structure. DFT calculations on $\mathrm{PdH}_{x}$ with $x \leq 1$ that neglected the $\mathrm{ZPE}$ showed that hydrogen prefers to occupy the tetrahedral sites as compared to the octahedral ones when the hydrogen content is large. $182[186$ This contradicts the results of most experimental observations, which are indicative of octahedral site filling. ${ }^{187}$ Inclusion of the ZPE was found to have a dramatic impact on the relative enthalpies of the zincblende, wurtzite and rock-salt structures, stabilizing the latter, $\frac{182}{12}$ as shown in Fig. 8 . The Pd-H distances are $\sim 0.25 \AA$ shorter in the $\mathrm{NaCl}$ structure, which leads to lower frequency vibrations that affect the total ZPE. At ambient conditions $\mathrm{PdH}$ is superconducting with a $T_{c}$ of 8-9 K. ${ }^{188}$ When hydrogen is replaced with deuterium the $T_{c}$ increases to $10-11 \mathrm{~K}$, the so-called "inverse isotope effect". $183 \mid 188$ The $T_{c}$ of both phases is reduced under pressure. ${ }^{189190}$ A tremendous amount of research has been devoted to uncovering the origin of the inverse isotope effect, and recently state-of-the-art first-principles calculations have illustrated that this phenomenon originates from the large anharmonicity in the phonon modes, $\frac{184}{25}$ see Fig. 8 . The superconductivity was shown to be phonon mediated, but neglecting the anharmonic motion leads to a large overestimation of the $T_{c}$. At pressures of $5 \mathrm{GPa}$ or less phase segregation into $\mathrm{PdH}$ and $\mathrm{Pd}_{3} \mathrm{H}_{4}$, resulting from the removal of $\mathrm{Pd}$ atoms, occurs. 191

The theoretical suggestion that compressed silane may be superconducting at pressures lower than those required to metallize hydrogen ${ }^{3 / 192 \mid 193}$ inspired a number of experiments. One of these concluded that metalization of $\mathrm{SiH}_{4}$ occurs at $50 \mathrm{GPa}$, and measured a $T_{c}<17 \mathrm{~K}$ at $96 \mathrm{GPa}$ and $120 \mathrm{GPa} .{ }^{194}$ However, discrepancies between theory and experiments (see Sec. 6 for further details) coupled with the realization that the platinum electrodes employed to measure the conductivity in the sample may react with hydrogen released from the pressure induced decomposition of $\mathrm{SiH}_{4}$, led to the suggestion that a superconducting hydride of platinum formed under pressure instead. ${ }^{146}$ CSP investigations showed that the PtH stoichiometry, which was found to be thermodynamically favored over the elemental phases between $3 \mathrm{GPa}^{162}$ to $20 \mathrm{GPa},{ }^{195}$ was the most stable point on the $\mathrm{Pt} / \mathrm{H}$ phase diagram at $\sim 100 \mathrm{GPa}{ }^{196}$ The two phases that were nearly isoenthalpic at $100 \mathrm{GPa}, \mathrm{hcp},{ }^{195[196}$ and $\mathrm{fcc}{ }^{195} \mathrm{PtH}$, shown in Fig. 9, were found to be superconducting. The highest $T_{c}$ occurred at the onset of dynamic stability, with fcc-PtH having a slightly larger value than hcp-PtH. ${ }^{195}$ The $T_{c}$ of both phases decreased with the application of pressure, 195 approaching $0 \mathrm{~K}$ by $\sim 200 \mathrm{GPa}$. The XRD pattern calculated for hcp-PtH matched well with the experimental results for 'silane', 196 as did the computed $T_{c}$ values. ${ }^{195 \mid 196198}$ Subsequent experiments confirmed the room-temperature synthesis of $\mathrm{PtH}$ above $27 \mathrm{GPa}$, and the formation of an hep structure above $42 \mathrm{GPa} \cdot{ }^{198}$ However, first principles computations that took anharmonic effects into account found the $T_{c}$ of hcp-PtH to be $<1 \mathrm{~K}$ at $100 \mathrm{GPa}{ }^{199}$ This strong suppression in $\lambda$ and in $T_{c}$ resulting from anharmonicity led the authors to question whether or not the superconductivity observed in experiment did in fact originate from $\mathrm{PtH}$.

\subsection{Group 11: Copper, Silver, Gold}

The first synthesis of copper hydride was by Wurtz in 1844,200 and it is the only coinage metal hydride that has been prepared to date. The protocol yielded a hcp crystal with the $\mathrm{CuH}$ stoichiometry that possessed the $P 6_{3} m c$ spacegroup, i.e. the wurtzite structure type. ${ }^{201202}$ This phase is stable below $-5^{\circ} \mathrm{C}$, but at room temperature it decomposes below $8.5 \mathrm{GPa} .{ }^{203}$ The synthesis of $\mathrm{CuH}$ above $14.4 \mathrm{GPa}$, on the other hand, yielded a $\mathrm{CuH}_{0.4}$ stoichiometry where the metal lattice 
(a)

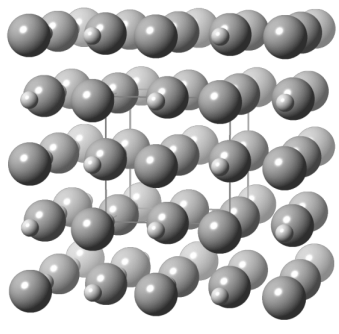

fcc PtH (b)

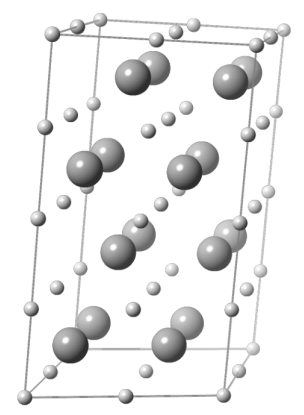

hcp PtH

Figure 9: Two PtH phases predicted to be stable under pressure using CSP. ${ }^{195] 196 / 198}$ These fcc ( $\mathrm{NaCl}$ structure with $F m \overline{3} m$ symmetry) and hcp ( $P 6_{3} / m m c$ symmetry) structures have been predicted or observed in many transition metal monohydrides. Calculations confirmed that both fcc and hcp PtH were superconducting. It has been proposed that one of these phases formed when the platinum comprising the electrodes employed in experiment reacted with the $\mathrm{H}_{2}$ that was liberated from the decomposition of compressed $\mathrm{SiH}_{4}$, and the $\mathrm{PtH}$ phase was responsible for the superconductivity observed in Ref. 194

is fcc and the hydrogen atoms reside in the interstitial regions $(\gamma-\mathrm{CuH}) .{ }^{204} \mathrm{~A}$ third phase, $\epsilon-\mathrm{Cu}_{2} \mathrm{H}$, that possesses an anti-CdI $\mathrm{C}_{2}$ structure ( $P \overline{3} m 1$ spacegroup) has been made in a DAC between 18.6$51 \mathrm{GPa} \cdot{ }^{205}$ The hydrogen atoms within this phase were found to be arranged in layers, instead of filling the void sites randomly. It is not clear why the method of preparation affects which hydride of copper is formed.

Experiments wherein silver and gold were compressed in a hydrogen medium up to $87 \mathrm{GPa}$ and $113 \mathrm{GPa}$, respectively, did not yield hydrides of these metals at, or above, room temperature. ${ }^{205}$ In another study, AuH was reportedly synthesized by annealing pure gold in a hydrogen atmosphere at $\sim 5 \mathrm{GPa}$ and $\sim 400^{\circ} \mathrm{C}$. ${ }^{[206}$ However, its structure is unknown, and the experiment has not been confirmed since. Based on the diffraction pattern, it was suggested that the structure may be related to the $t I 2$ phase of mercury. ${ }^{207}$ Theoretical work has therefore been undertaken to determine if hydrides of the heavier coinage metals could be synthesized. DFT calculations on $\mathrm{AgH}$ phases assuming structures that were predicted to be metastable or stable for $\mathrm{PtH}$ and $\mathrm{RuH}$ wherein different tetrahedral and octahedral sites were populated with hydrogen atoms suggested that $\mathrm{AgH}$ would become stable above $180 \mathrm{GPa} \cdot \frac{162}{162}$ The most stable structure was fcc with hydrogen occupying the octahedral sites. At $100 \mathrm{GPa}$ AgH was calculated to be a poor metal, suggesting that it is unlikely to be a high temperature superconductor. Another investigation found that dynamic stability within fcc-AgH was achieved by $50 \mathrm{GPa}$, and at this pressure the system was a semi-conductor precluding it from superconductivity. ${ }^{[195]}$ The theoretical calculations carried out so far do not support the stability of the monohydride of gold at the low pressures employed in Ref. ${ }^{206}$ For example, AuH in the fcc structure was computed to become dynamically stable above $220 \mathrm{GPa} \cdot{ }^{195}$ And, in a subsequent DFT study none of the structure types that were considered for $\mathrm{AuH}$ were found to be thermodynamically stable with respect to the elemental phases at pressures attainable in a DAC. 162 


\subsection{Group 12: Zinc, Cadmium, Mercury}

At ambient conditions in the solid state the group 12 metals form dihydride phases. It is believed that $\mathrm{HgH}_{2}$ is a covalent molecular solid, ${ }^{208}$ whereas $\mathrm{ZnH}_{2}$ and $\mathrm{CdH}_{2}$ contain hydrogen bridges between the metal atoms. Because solid $\mathrm{HgH}_{2}$ and $\mathrm{CdH}_{2}$ decompose to the elemental phases at low temperatures, they have not been intensely investigated. ${ }^{209}$ At the time of writing this review, we were unable to locate any theoretical or experimental studies that had examined the structures and superconducting properties of the hydrides of zinc, cadmium or mercury as a function of pressure.

\section{Group 13: Icosagen Hydrides}

\section{Boron}

Even though the chemistry of boron hydride clusters has been actively researched, $\stackrel{210}{20}$ not as much effort has been placed into studying these systems in the solid state and under pressure. One exception is diborane, $\mathrm{B}_{2} \mathrm{H}_{6}$. At ambient conditions molecular diborane assumes a geometry wherein two hydrogens each bridge two boron atoms, and it is metastable towards hydrogen loss. Crystalline $\mathrm{BH}_{3}$ is unknown, however solid diborane adopts the $\alpha$ phase below $60 \mathrm{~K}$, and annealing above $90 \mathrm{~K}$ yields the $\beta$ phase shown in Fig. 10(a). Raman spectroscopy has been employed to study diborane up to $24 \mathrm{GPa} \cdot{ }^{211}$ At $4 \mathrm{GPa}$ the system underwent a liquid-solid transition to phase I, followed by a transformation to phase II at $6 \mathrm{GPa}$, and phase III at $14 \mathrm{GPa}$. The phase transitions were reversible upon decompression. Infrared (IR) spectroscopy studies up to $50 \mathrm{GPa}$ provided further evidence for the phase transitions observed via Raman. ${ }^{212}$ The IR measurements suggested that the $\mathrm{B}_{2} \mathrm{H}_{6}$ molecule remains intact within these phases. Spectroscopic studies also provided evidence for further structural transitions at $42 \mathrm{GPa}$ and $57 \mathrm{GPa} .213$ Another boron hydride whose high pressure behavior has been scrutinized experimentally is decaborane, $\mathrm{B}_{10} \mathrm{H}_{14} \cdot{ }^{214}$ It's Raman spectrum did not show any dramatic changes up to $50 \mathrm{GPa}$. Above this pressure the sample changed color from transparent yellow to orange/red, and Raman spectroscopy suggested that the backbone of the molecule had been perturbed. The sample became black above $100 \mathrm{GPa}$ implying that a transition into a non-molecular phase, which was shown to be semiconducting, had occurred.

Early theoretical studies concluded that molecular boranes become thermodynamically unstable towards systems comprised of extended bonded networks by $\sim 100-300 \mathrm{GPa}$, and that a $\mathrm{BH}_{3}$ analogue of $\mathrm{AlH}_{3}$ would become metallic below $30 \mathrm{GPa} \cdot{ }^{215}$ Twenty years later DFT cal-

culations ${ }^{213 / 216}$ were carried out to help characterize the phases investigated in Refs. $\frac{211212}{2}$ The computed IR and Raman spectra of ten candidate phases that contained the molecular diborane unit were compared with those obtained experimentally. ${ }^{216}$ This study showed that phase I corresponds to the $\beta$-diborane structure, and the best candidates for phases II and III possessed a $P 2_{1} / c$ symmetry lattice, but with different molecular orientations. Importantly, all of the experimental data was consistent with phases containing $\mathrm{B}_{2} \mathrm{H}_{6}$ units, suggesting that transformation to the thermodynamically preferred products, cyclic oligomers and polymer chains, is kinetically hindered. Further theoretical studies concluded that the phases observed at $42 \mathrm{GPa}$ and $57 \mathrm{GPa}$ possessed $P 1$ symmetry and were comprised of $\mathrm{B}_{2} \mathrm{H}_{6}$ molecules. ${ }^{[13}$ Moreover, a geometry optimization of the proposed phase IV structure showed that near $110 \mathrm{GPa}$ the molecular diborane motifs poly- 
merize forming a phase containing one-dimensional zig-zag chains of boron atoms. This phase was found to become metallic near $138 \mathrm{GPa}$ within hybrid DFT. Another theoretical investigation carried out at about the same time showed that crystals based upon $\mathrm{B}_{3} \mathrm{H}_{9}$ trimers become stabilized with respect to $\beta$-diborane between 4-36 GPa, and at higher pressures linear polymers become preferred. ${ }^{217}$ Two of the proposed structures are illustrated in Fig. 10.(b) and Fig. 10.c). The kinetic barrier for trimer formation was estimated to be large, and it was therefore concluded that it is unlikely that such phases were made in Refs. ${ }^{2111212}$ The calculations also showed that the structural interconversion between $\beta-\mathrm{B}_{2} \mathrm{H}_{6}$ and a $P 2_{1} / c$ symmetry polymeric phase was likely to be facile, suggesting that the latter could be a candidate for the experimentally observed phase III. None of the phases studied were found to be metallic. Another computational study showed that phases with the $\mathrm{B}_{2} \mathrm{H}_{6}$ stoichiometry become thermodynamically stable with respect to segregation into the elemental structures above $350 \mathrm{GPa} \cdot{ }^{218}$ Two metallic systems, one with Pbcn and the other with $\mathrm{Cmcm}$ symmetry were predicted, however the latter was not dynamically stable within the harmonic approximation. At $360 \mathrm{GPa}$ the $T_{c}$ of the Pbcn phase shown in Fig. 10.d) was estimated as being $125 \mathrm{~K}$.

(a)

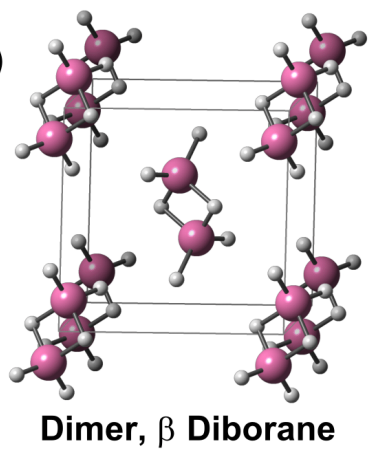

(c)

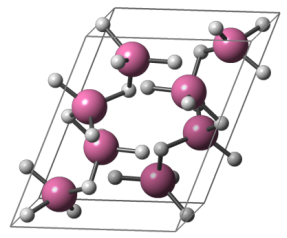

Polymer, $P 2_{1} / c$

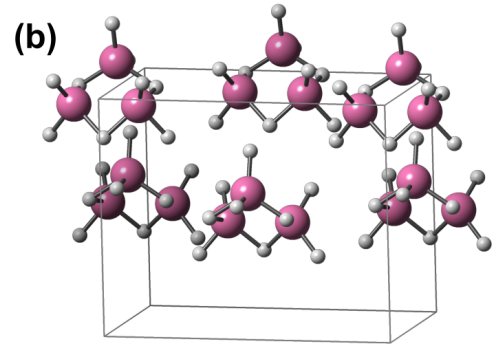

Trimer, $\mathrm{Cmc2}_{1}$

(d)

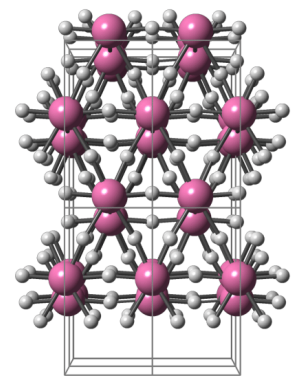

Pbcn

Figure 10: (a) The $\beta$-diborane phase that forms above $90 \mathrm{~K}$ at atmospheric pressures. Predicted high pressure phases of $\mathrm{BH}_{3}$ including: (b) one that contains $\mathrm{B}_{3} \mathrm{H}_{9}$ trimers, ${ }^{217}$ (c) one that consists of linear $\left(\mathrm{BH}_{3}\right)_{n}$ polymeric chains,,$\frac{217}{}$ and (d) a $\mathrm{Pbcn}$ phase whose $T_{c}$ was estimated as being $125 \mathrm{~K}$ at $360 \mathrm{GPa} .218$

Finally, CSP techniques have been employed to predict the most stable structures with the $\mathrm{BH}$ and $\mathrm{BH}_{3}$ stoichiometries up to $300 \mathrm{GPa}, \stackrel{219}{2}$ and the $\mathrm{B}_{4} \mathrm{H}_{10}, \mathrm{~B}_{4} \mathrm{H}_{8}$ and $\mathrm{B}_{4} \mathrm{H}_{6}$ stoichiometries between $50-300 \mathrm{GPa} \cdot \stackrel{220}{ }$ In Ref. ${ }^{219}$ the $\mathrm{BH}$ stoichiometry was found to be stable above $50 \mathrm{GPa}$, 
and it was the only boron hydride phase thermodynamically favorable above $153 \mathrm{GPa}$. Below $175 \mathrm{GPa}$ the preferred $\mathrm{BH}$ phases were composed of hydrogen-terminated puckered boron sheets, and a three-dimensional $P 6 / \mathrm{mmm}$ symmetry structure that was metallic with an estimated $T_{c}$ of $14-21 \mathrm{~K}$ at $175 \mathrm{GPa}$ was stable at higher pressures. Ref. ${ }^{220}$ found that the lowest enthalpy $\mathrm{B}_{4} \mathrm{H}_{10}$ stoichiometry phases at 50 and $150 \mathrm{GPa}$ consisted of hydrogen-capped boron layers separated by $\mathrm{H}_{2}$ units, suggesting that segregation into the elemental phases is preferred at these pressures. At $300 \mathrm{GPa}$ a three-dimensional network was predicted to be the most stable. With few exceptions, the structures predicted for $\mathrm{B}_{4} \mathrm{H}_{8}$ and $\mathrm{B}_{4} \mathrm{H}_{6}$ consisted of molecular or polymeric units that did not undergo phase segregation. The reaction $\mathrm{B}_{4} \mathrm{H}_{10} \rightarrow \mathrm{B}_{4} \mathrm{H}_{8}+\mathrm{H}_{2}$ was found to be exothermic at the pressures considered.

\section{Aluminum}

In the gas phase a number of molecular hydrides of aluminum, including $\mathrm{AlH}_{n}(n=1-3)$, $\mathrm{Al}_{2} \mathrm{H}_{4}$ and $\mathrm{Al}_{2} \mathrm{H}_{6}$ have been formed via laser ablation. ${ }^{221}$ In the solid state, however, only the $\mathrm{AlH}_{3}$ compound is known, and at atmospheric conditions it is metastable releasing $\mathrm{H}_{2}$ molecules when heated. $\frac{222}{\text { Solid }} \mathrm{AlH}_{3}$ can adopt one of four different modifications depending upon the method of synthesis. According to DFT calculations at $0 \mathrm{~K}$ the $\beta$ phase has the lowest energy at atmospheric pressures, and it transitions to the $\alpha^{\prime}$ and $\alpha$ structures at 2.4 and $4.3 \mathrm{GPa} \cdot{ }^{[23} \mathrm{A}$ number of theoretical $[23+226$ and experimental $226-231$ studies have focused on the structural transitions and properties of $\mathrm{AlH}_{3}$ under pressure.

At 1 atm $\mathrm{AlH}_{3}$ has a large band gap, like other ionic solids. However, because this high hydrogen content material should become metallic via pressure induced band broadening, it was suggested that $\mathrm{AlH}_{3}$ might become superconducting when squeezed. Ab Initio random structure searches predicted that the $\alpha$ phase would transition to an insulating layered Pnma structure at $34 \mathrm{GPa}{ }^{232}$ At $73 \mathrm{GPa}$ a transformation to the semi-metallic $P m \overline{3} n$ symmetry structure shown in the inset of Fig. 11 was found. Both of these phases were stable with respect to dehydrogenation under pressure. A later theoretical study showed that $P m \overline{3} n-\mathrm{AlH}_{3}$ is dynamically stable between 72-106 GPa at $0 \mathrm{~K}$, as well as at $1 \mathrm{~atm}$ and $\sim 470 \mathrm{~K} \cdot{ }^{[233}$ Within PBE the metallicity of $P m \overline{3} n$ $\mathrm{AlH}_{3}$ was found to arise from the conduction and valence bands crossing the Fermi level at the $R$ and $M$ points, respectively, as shown by the red bands in Fig. 11. The DOS at $E_{F}$ decreased with increasing pressure, and GW calculations showed that this phase becomes insulating by $200 \mathrm{GPa}$ because the center of the $\mathrm{Al} 3 s$ band and the $\mathrm{H} 1 s$ band become increasingly separated with pressure. ${ }^{234}$ At $72 \mathrm{GPa}$ the $T_{c}$ of $P m \overline{3} n-\mathrm{AlH}_{3}$ was estimated as being $11 \mathrm{~K}$ via the modified McMillan equation, and $T_{c}$ decreased with increasing pressure, approaching zero by $165 \mathrm{GPa} .235$

The aforementioned predictions inspired a combined theoretical/experimental study. ${ }^{[237} \alpha$ $\mathrm{AlH}_{3}$, which adopts the $R \overline{3} c$ structure, was found to undergo a structural transition to an unknown phase at $63 \mathrm{GPa}$. At $100 \mathrm{GPa}$ a transition to a phase that could be indexed to either the $\operatorname{Im} \overline{3} \mathrm{~m}$ or the $P m \overline{3} n$ spacegroup, which differ only in the positions of the hydrogen atoms, occurred. Resistance measurements were indicative of an insulator to metal transition, but superconductivity was not observed up to $165 \mathrm{GPa}$ at temperatures as low as $4 \mathrm{~K}$. DFT calculations showed that the $\operatorname{Pm} \overline{3} n$ structure is enthalpically preferred, and this metallic phase was calculated to have a $T_{c}$ of $24 \mathrm{~K}$ at $110 \mathrm{GPa}$ and $6 \mathrm{~K}$ at $165 \mathrm{GPa}$. However, the reason for the discrepancy in the superconducting properties between experiment and theory could not be found. A later computational study revealed that a large fraction of the electron-phonon coupling in this phase arises 


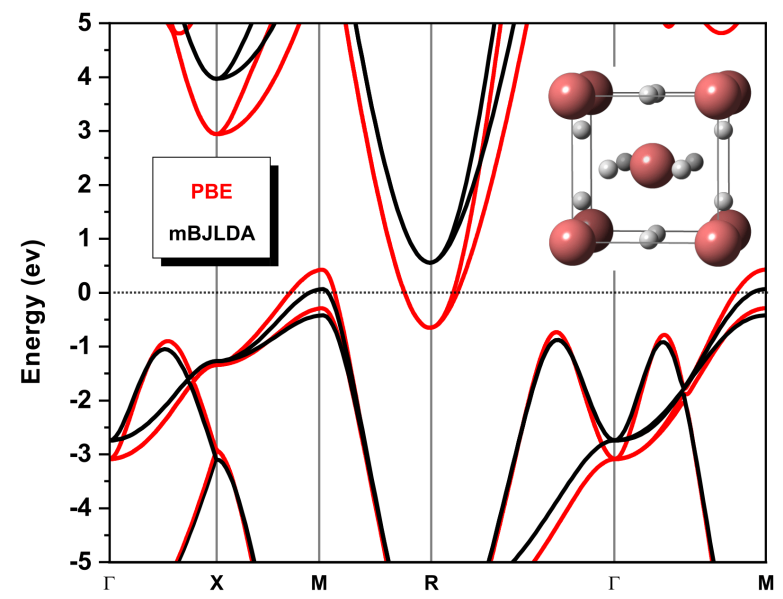

Figure 11: The band structure of the $P m \overline{3} n-\mathrm{AlH}_{3}$ phase shown in the inset as calculated with the $\mathrm{PBE}$ and TB-mBJLDA functionals at $160 \mathrm{GPa} .{ }^{236}$

from modes that are highly anharmonic, and when the anharmonicity was taken into account the electron-phonon coupling, and therefore $T_{c}$, was greatly diminished. ${ }^{238 / 239}$ Another theoretical study illustrated that the band structure near the Fermi level, and in particular bands that give rise to nested pieces of Fermi surface that are integral for the electron-phonon coupling, depend upon the computational method employed. ${ }^{236}$ In contrast to calculations performed with the PBE functional, both the TB-mBJLDA functional and GW calculations predicted that at $160 \mathrm{GPa}^{\mathrm{AlH}} 3$ should be a small gap semi-conductor, as shown by the black bands in Fig. 11. Thus, the disagreement between experiment and theory might be due to a number of factors that were not considered in the original calculations, such as the anharmonicity of the phonon modes or the way in which exact exchange influences the band structure around the Fermi level.

CSP techniques have been employed to predict the structures adopted by $\operatorname{AlH}_{n}(n=5,7,9)$ up to $300 \mathrm{GPa} \cdot{ }^{240}$ The most stable phase below $73 \mathrm{GPa}, P 1-\left(\mathrm{AlH}_{3}\right) \mathrm{H}_{2}$, could be thought of as a vdW compound. Pressure was found to induce a transformation to a semiconducting $P 1$ symmetry phase, which had the lowest enthalpy of any $\mathrm{AlH}_{5}$ configuration examined below $250 \mathrm{GPa}$. Above this pressure a metallic $P 2_{1} / m-\mathrm{AlH}_{5}$ structure became preferred, and its large electronphonon coupling resulted in a $T_{c}$ of $132-146 \mathrm{~K}$.

\section{Gallium}

A number of molecular hydrides of gallium including $\mathrm{GaH}, \mathrm{GaH}_{2}, \mathrm{GaH}_{3}$, and $\mathrm{Ga}_{2} \mathrm{H}_{2},{ }^{241}$ have been prepared in solid noble gas matrices. In addition, wet chemical methods have been employed to synthesize the bridge-bonded molecule digallane, $\mathrm{H}_{2} \mathrm{Ga}(\mu-\mathrm{H})_{2} \mathrm{GaH}_{2} \cdot{ }^{242}$ This highly reactive compound ${ }^{243}$ condenses as a white solid, which may have a polymeric structure, and it decomposes into the elements above $243 \mathrm{~K}$. On the theoretical front, computations have been undertaken to predict the most stable solid state structures of $\mathrm{GaH}_{3}$ between 5-300 GPa. ${ }^{244}$ At 
low pressures the preferred systems contained $\mathrm{H}_{2}$ units, suggesting that they are prone towards decomposition. Above $160 \mathrm{GPa}$ a $\operatorname{Pm} \overline{3} n$ phase with monoatomic hydrogen atoms, which is isotypic with the high pressure form of $\mathrm{AlH}_{3}$ shown in the inset of Fig. 111, was computed to become stable with respect to the elemental phases. The $T_{c}$ of this ionic solid was estimated as being 76$83 \mathrm{~K}$ at $160 \mathrm{GPa}$ via the Allen-Dynes modified McMillan equation, and it was found to decrease with increasing pressure. At $120 \mathrm{GPa}$ the $T_{c}$ of $P m \overline{3} n-\mathrm{GaH}_{3}$ calculated by solving the Eliashberg equations ranged from $90-123 \mathrm{~K} .245$

\section{Indium and Thallium}

Despite claims whose origins date back over 60 years, it is unlikely that the trihydrides of indium and thallium were ever successfully synthesized. ${ }^{246}$ In fact, some studies suggest that $\mathrm{InH}_{3}$ and $\mathrm{TlH}_{3}$ are not stable enough to be isolated in the solid state at ambient pressure and temperature. ${ }^{247 / 248}$ However, molecular hydrides of these elements have been synthesized in the gas phase at cryogenic temperatures via laser ablation in inert matrices. These include $\operatorname{In}_{2} \mathrm{H}_{2}, \mathrm{InH}_{3}, \mathrm{In}_{2} \mathrm{H}_{4}$, $\mathrm{In}_{2} \mathrm{H}_{6},{ }^{241|248| 249} \mathrm{TlH}, \mathrm{TlH}_{2}, \mathrm{TlH}_{3}, \mathrm{Tl}_{2} \mathrm{H}_{2}$ and $\mathrm{TlTlH}_{2} \cdot{ }^{250} \mathrm{CSP}$ techniques have therefore been used to determine if hydrides of indium could become stable under pressure. ${ }^{251} \mathrm{InH}_{3}$ and $\mathrm{InH}_{5}$ became enthalpically preferred over the elemental phases by $200 \mathrm{GPa}$. They contained $\mathrm{H}_{2}$ or linear $\mathrm{H}_{3}$ units, wherein charge was donated from the indium to the hydrogen atoms, and at $200 \mathrm{GPa}$ their $T_{c}$ values were estimated as being $34-40 \mathrm{~K}$ and $22-27 \mathrm{~K}$, respectively. With increasing pressure, the $T_{c}$ of $R \overline{3}-\mathrm{InH}_{3}$ decreased slightly. To the best of our knowledge CSP studies on $\mathrm{TlH}_{n}$ have not yet been carried out. But, based upon the trends observed for the icosagen hydrides we suspect that the hydrides of thallium become stable at pressures larger than $200 \mathrm{GPa}$.

\section{Group 14: Tetragen Hydrides}

\section{Carbon}

It is beyond the scope of this review to discuss all of the work that has been carried out on compressed solids containing hydrocarbon based molecules. Instead, we briefly describe the high pressure behavior of methane and methane/hydrogen mixtures, which have been intensely studied because of their relevance in planetary sciences. Methane has a rich phase diagram, 252 and various binary molecular compounds with the general formula $\left(\mathrm{CH}_{4}\right)_{n}\left(\mathrm{H}_{2}\right)_{m}(n=1,2$ and $m=1,2,4)$ have been characterized spectroscopically up to $30 \mathrm{GPa} \cdot{ }^{253} \mathrm{~A}$ wide variety of CSP techniques have been used to predict the phases methane adopts under pressure, but none of them found any stable metallic structures up to pressures as high as $550 \mathrm{GPa}{ }^{254} \mathrm{CSP}$ investigations have also suggested that both methane ${ }^{255 \mid 258}(95-200 \mathrm{GPa})$ and a 1:1 mixture of $\mathrm{CH}_{4}$ and $\mathrm{H}_{2}{ }^{259}$ ( $P>230 \mathrm{GPa}$ ) become thermodynamically unstable towards decomposition into other hydrocarbon based phases by the pressures given in the parentheses, even though they may remain dynamically stable. The lowest enthalpy systems were found to be large band gap insulators to at least $150 \mathrm{GPa}{ }^{259}$ These theoretical studies suggest that it is unlikely that a methane-based hydride could exhibit superconductivity at pressures that are currently accessible via static compression.

\section{Silicon}

In 2004 Neil Ashcroft proposed that the same attributes that would render metallic hydrogen 
a high temperature superconductor would be applicable to hydrogen dominant alloys, and in particular those containing a group 14 element such as silicon. ${ }^{3 / 4}$ He also predicted that the group 14 hydrides would become metallic at pressures lower than those required to metallize elemental hydrogen because of "chemical precompression". These predictions inspired numerous studies of the hydrides of silicon under pressure. In the first study, carried out in 2006 by Feng et al., DFT calculations were performed on 13 candidate $\mathrm{SiH}_{4}$ structures. ${ }^{192}$ The most stable phase underwent pressure induced band gap closure just under $100 \mathrm{GPa}$, and it was suggested that it might be a high temperature superconductor. A subsequent study by Pickard and Needs, which used random searching instead, found a $I 4_{1} / a$ phase between 50-263 GPa and a $C 2 / c$ phase at higher pressures - these phases were more stable than the structures considered by Feng and co-workers. ${ }^{193} I 4_{1} / a$-SiH 4 , shown in Fig. 12 (a), underwent band gap closure at $200 \mathrm{GPa}$. C2/c$\mathrm{SiH}_{4}$, shown in Fig. 12 (b), was a good metal, suggestive of high temperature superconductivity. Simulated annealing was also employed to investigate the behavior of silane under pressure. 260 A metallic $C 2 / c$ symmetry phase, which differed from the one predicted by Pickard and Needs, was singled out for further investigation. Despite the fact that it had an enthalpy higher than that of $I 4_{1} / a-\mathrm{SiH}_{4}$, it was found to be dynamically stable between 65-150 GPa. The Allen-Dynes modified McMillan equation yielded estimates of $45-55 \mathrm{~K}$ for the $T_{c}$ of the metastable $C 2 / c$ $\mathrm{SiH}_{4}$ phase at $125 \mathrm{GPa}$, whereas $I 4_{1} / a-\mathrm{SiH}_{4}$ was not a superconductor at $150 \mathrm{GPa}$. A combined experimental and theoretical study proposed that $\mathrm{SiH}_{4}$ assumes the $P 2_{1} / c$ symmetry spacegroup between 10-25 GPa. ${ }^{261}$ Within this pressure range the enthalpy of this phase was found to be lower than any of the previously proposed systems. Experiments showed the emergence of a new phase around $27 \mathrm{GPa}$, and theoretical work suggested that the structure formed might be the polymeric $F d d 2$ symmetry phase illustrated in Fig. 12.(c).

Meanwhile, optical experiments suggested that silane undergoes an insulator-semiconductor phase transition around $100 \mathrm{GPa}$, but metalization did not occur below $210 \mathrm{GPa} .{ }^{266}$ A comprehensive experimental study of $\mathrm{SiH}_{4}$ showed no evidence for metalization to at least $150 \mathrm{GPa}$, at which pressure the band gap was estimated as being 0.6-1.8 eV. ${ }^{267}$ On the other hand, Raman and infrared spectroscopy detected three phase transitions below $30 \mathrm{GPa}$, and reflectivity measurements suggested the onset of metalization above $60 \mathrm{GPa} .{ }^{268}$ Remarkably, resistance measurements showed that silane metalized at $50 \mathrm{GPa}$ and superconductivity with $T_{c}=17 \mathrm{~K}$ was observed at $96 \mathrm{GPa}$ and $120 \mathrm{GPa} \cdot{ }^{194}$ Based upon XRD, a $P 6_{3}$ symmetry structure was proposed for the superconducting phase. Above $120 \mathrm{GPa}$ an insulating transparent phase, whose diffraction pattern matched with the one obtained for the $I 4_{1} / a-\mathrm{SiH}_{4}$ structure of Pickard and Needs, formed. A few years later this polymeric $I 4_{1} / a-\mathrm{SiH}_{4}$ phase was synthesized at $124 \mathrm{GPa}$ and $300 \mathrm{~K} .262$

Further theoretical studies found that the superconducting phase synthesized in Ref. ${ }^{194}$ could not possess the proposed $\mathrm{P}_{3}$ symmetry structure, since it was found to be dynamically unstable, and its enthalpy was significantly higher than that of other alternatives. ${ }^{269}-272$ Computations suggested that metastable structures with $\mathrm{Cmca},{ }^{269} \mathrm{Pbcn}{ }^{271}$ or $\mathrm{P} 4 / \mathrm{nbm}^{270}$ symmetries could be candidates for the superconducting phase. Eliashberg theory was employed to calculate the superconducting properties of some of these phases. ${ }^{273+275]}$ However, none of the proposed structures could fully explain the experimental results. Because of this, it has been suggested that the measured superconductivity originated from unintended reaction products formed from the decomposition of silane under pressure, as described in Sec. 4.8 . 
(a)
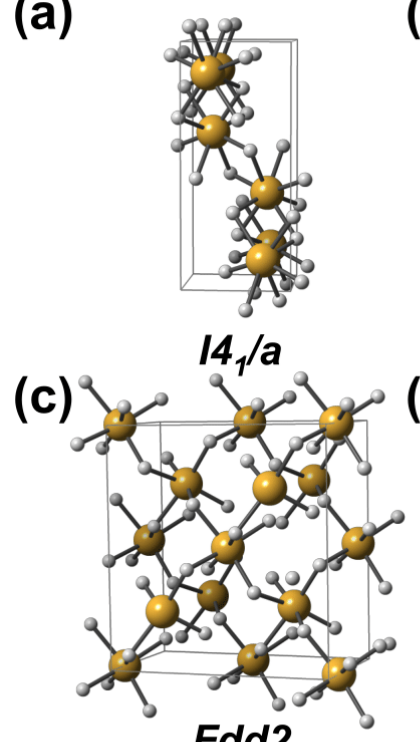

(e)

Fdd2

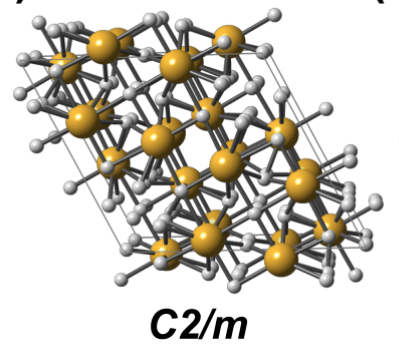

(b)

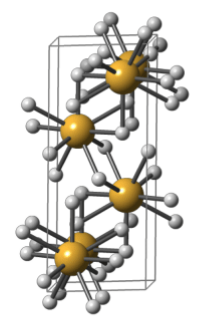

C2/c

(d)

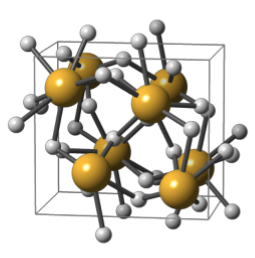

(f)

$P 2_{1} / C$

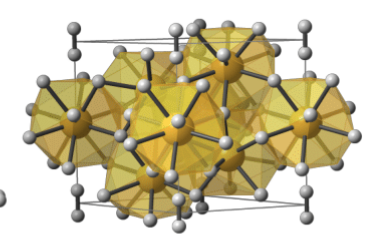

$P \overline{3}$

Figure 12: Various $\mathrm{SiH}_{4}$ phases that have been investigated under pressure: (a) an $I 4_{1} / a$ symmetry phase that was theoretically predicted ${ }^{193}$ and experimentally synthesized, ${ }^{262}$ (b) a theoretically predicted $C 2 / c$ phase, ${ }^{193}$ (c) a theoretically predicted $F d d 2$ phase, ${ }^{263}$ which is a likely candidate for an experimentally observed polymeric phase, (d) a theoretically predicted $P 2_{1} / c$ phase with $T_{c}=32 \mathrm{~K}$ at $400 \mathrm{GPa},{ }^{264}$ (e) a theoretically predicted $C 2 / m$ phase with $T_{c}=106 \mathrm{~K}$ at $610 \mathrm{GPa},{ }^{264}$ (f) a theoretically predicted $P \overline{3}$ phase with $T_{c}=32 \mathrm{~K}$ at $300 \mathrm{GPa} .{ }^{265}$

CSP techniques have recently been employed to explore the phases that silane adopts at significantly higher pressures than those considered previously, some of these are illustrated in Figs. 12 (c-f). One of the phases found possessed $P 2_{1} / c$ symmetry above $383 \mathrm{GPa}$, and another $C 2 / \mathrm{m}$ symmetry above $606 \mathrm{GPa}$ with estimated $T_{c}$ values of $32 \mathrm{~K}$ at $400 \mathrm{GPa}$, and $106 \mathrm{~K}$ at $610 \mathrm{GPa}$, respectively. $\frac{264}{}$ Another study found a $P \overline{3}$ symmetry phase, which could be described as a polymeric $\mathrm{Si}-\mathrm{H}$ structure intercalated with $\mathrm{H}_{2}$ units whose $T_{c}$ was $32 \mathrm{~K}$ at $300 \mathrm{GPa}$, to be the most stable alternative above $241 \mathrm{GPa} .265$

Mixtures of silane and molecular hydrogen have also been intensely investigated both experimentally and theoretically. Spectroscopic evidence for the formation of compounds with the general formula $\mathrm{SiH}_{4}\left(\mathrm{H}_{2}\right)_{n}$ at $6.5-35 \mathrm{GPa}$ was obtained nearly simultaneously by two different groups. $\frac{138 \mid 276}{}$ First principles calculations $\frac{277.280}{280}$ have studied potential candidates for the 
$\mathrm{SiH}_{4}\left(\mathrm{H}_{2}\right)_{2}$ phase synthesized by Strobel and co-workers. ${ }^{138}$ CSP at higher pressures predicted that the $\mathrm{Ccca}-\mathrm{SiH}_{4}\left(\mathrm{H}_{2}\right)_{2}$ phase illustrated in Fig. 13(a) would become stable with respect to the elements above $248 \mathrm{GPa}$, and its $T_{c}$ was estimated to be $98-107 \mathrm{~K}$ at $250 \mathrm{GPa} \cdot{ }^{281}$ Its superconducting properties have subsequently been examined using the Eliashberg formalism. $282[283$

The experimental availability of the $\mathrm{Si}_{2} \mathrm{H}_{6}$ molecule at standard conditions inspired CSP calculations on this stoichiometry. It was shown that disilane becomes stable with respect to decomposition into the elements at $135 \mathrm{GPa} \cdot{ }^{284}$ Above this pressure phases with $P \overline{1}, P m \overline{3} m$, and $C 2 / c$ symmetries with estimated $T_{c}$ values of $65-76 \mathrm{~K}$ at $175 \mathrm{GPa}, 139-153 \mathrm{~K}$ at $275 \mathrm{GPa}$ and $34-42 \mathrm{~K}$ at $300 \mathrm{GPa}$, respectively, were predicted as being stable. A later study found that disilane is thermodynamically unstable towards decomposition into $\mathrm{SiH}_{4}$ and the elemental phases below $190 \mathrm{GPa} \cdot{ }^{285}$ Above this pressure a $\mathrm{Cmcm}$ symmetry phase was found to have the lowest enthalpy up to $280 \mathrm{GPa}$, and its $T_{c}$ was estimated as being $20 \mathrm{~K}$ at $100 \mathrm{GPa}$ and $13 \mathrm{~K}$ at $220 \mathrm{GPa}$.

\section{Germanium}

The high pressure behavior of germane, $\mathrm{GeH}_{4}$, has also been intensely investigated. A theoretical study that considered germanium analogues of previously proposed candidate $\mathrm{SiH}_{4}$ and $\mathrm{CH}_{4}$ phases predicted that an insulating fcc structure was preferred below, and a metallic $\mathrm{SnF}_{4}$-like structure was preferred above $72 \mathrm{GPa} \cdot{ }^{286}$ Another study employing a similar approach concluded that germane would metallize at a pressure lower than silane. ${ }^{287}$ However, an evolutionary algorithm based investigation predicted phases whose enthalpies were significantly lower. ${ }^{\sqrt[288]{ }} \mathrm{In}$ this study it was also shown that solid germane is thermodynamically unstable with respect to decomposition into the elemental phases below $196 \mathrm{GPa}$. Above this pressure a metallic $C 2 / c$ symmetry phase, which contained $\mathrm{H}_{2}$ motifs with elongated bonds, was stable, and its $T_{c}$ was estimated as being $64 \mathrm{~K}$ at $220 \mathrm{GPa}$. The superconducting properties of $C 2 / c-\mathrm{GeH}_{4}$ were subsequently analyzed. 289

Even though germane is thermodynamically unstable at atmospheric conditions it does not decompose, implying that metastable phases may be accessible under pressure. Therefore, a theoretical study was carried out to find the most stable phases containing intact $\mathrm{GeH}_{4}$ units. ${ }^{290}$ The following set of pressure induced transitions were proposed: $P 2_{1} / c \rightarrow C m m m \rightarrow P 2_{1} / m \rightarrow$ $C 2 / c$. The metastable $C m m m-\mathrm{GeH}_{4}$ phase was predicted to have a $T_{c}$ of $40 \mathrm{~K}$ at $20 \mathrm{GPa}, \stackrel{291}{ }$ and its superconducting properties have been studied in greater detail. ${ }^{292}$ Recently, CSP techniques have predicted two hitherto unknown $\mathrm{GeH}_{4}$ phases, one with $\mathrm{Ama} 2$ symmetry at $250 \mathrm{GPa}$ and one with $C 2 / c$ symmetry at $500 \mathrm{GPa}$, to be thermodynamically, mechanically and dynamically stable with estimated $T_{c}$ values of 47-57 and 70-84 K, respectively. ${ }^{293}$

Spectroscopic evidence has shown that germane and $\mathrm{H}_{2}$ can form a compound with the approximate $\mathrm{GeH}_{4}\left(\mathrm{H}_{2}\right)_{2}$ stoichiometry at pressures of $7.5-27 \mathrm{GPa} \cdot{ }^{297}$ The $\mathrm{GeH}_{4}$ molecules within this phase are rotationally disordered and occupy fcc sites, whereas the $\mathrm{H}_{2}$ molecules fill both octahedral and tetrahedral lattice sites. These experiments inspired theoretical studies on phases with the $\mathrm{GeH}_{4}\left(\mathrm{H}_{2}\right)_{2}$ stoichiometry up to center of the earth pressures. ${ }^{294}$ Structures with $I \overline{4} m 2$ and $P m n 2_{1}$ symmetry, that differed only in the orientation of the $\mathrm{H}_{2}$ units, were proposed as the most likely candidates for the experimentally observed phases. Below $220 \mathrm{GPa}$ these phases were thermodynamically unstable. Above this pressure a stable, metallic $P 2_{1} / c$ symmetry phase (see Fig. 13(b)) with a $T_{c}$ of $76-90 \mathrm{~K}$ at $250 \mathrm{GPa}$ was found. The superconducting properties of $P 2_{1} / c-\mathrm{GeH}_{4}\left(\mathrm{H}_{2}\right)_{2}$ have been investigated, ${ }^{298}$ and its $T_{c}$ was found to decrease with increasing 
(a)

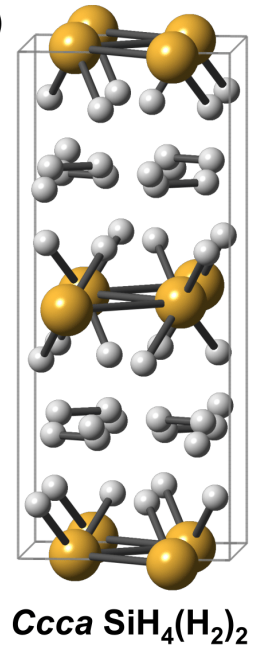

(b)

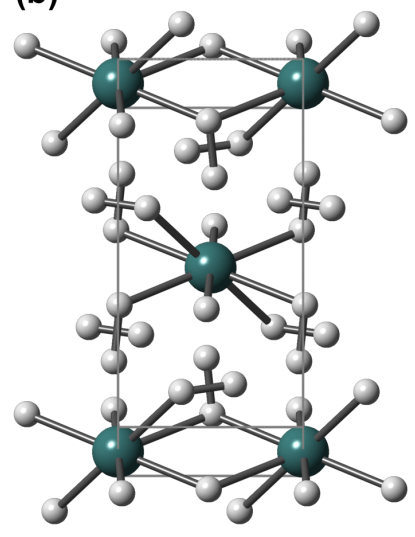

$P 2_{1} / c \mathrm{GeH}_{4}\left(\mathrm{H}_{2}\right)_{2}$ (c)

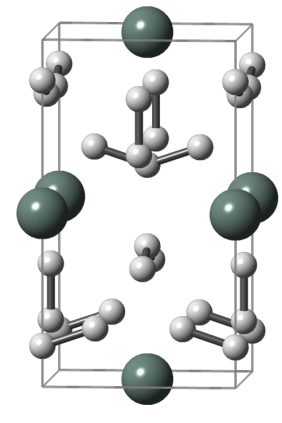

$\overline{14} \mathrm{~m} 2 \mathrm{SnH}_{8}$ (d)

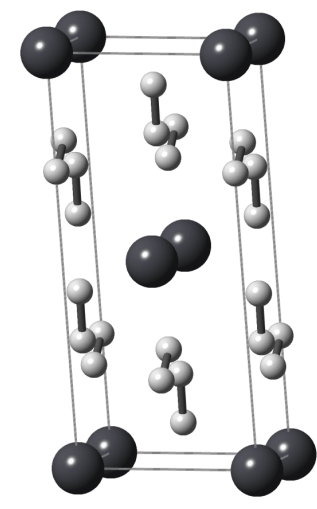

$\mathrm{C} 2 / \mathrm{m} \mathrm{PbH}_{4}\left(\mathrm{H}_{2}\right)_{2}$

Figure 13: Unit cells of superconducting phases predicted for group 14 hydrides with the $\mathrm{MH}_{4}\left(\mathrm{H}_{2}\right)_{2}$ stoichiometry: (a) $\mathrm{Ccca}-\mathrm{SiH}_{4}\left(\mathrm{H}_{2}\right)_{2}$ phase with $T_{c}=98-107 \mathrm{~K}$ at $250 \mathrm{GPa}, 281$ (b)

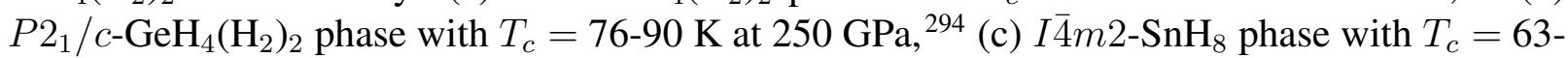
$72 \mathrm{~K}$ at $250 \mathrm{GPa}{ }^{295}$ and (d) $C 2 / m-\mathrm{PbH}_{4}\left(\mathrm{H}_{2}\right)_{2}$ phase with $T_{c} \approx 107 \mathrm{~K}$ at $230 \mathrm{GPa} \cdot{ }^{296}$

pressure. 299

Evolutionary algorithms have also been employed to search for the most stable binary compounds of germanium and hydrogen over a wide composition range. ${ }^{300 / 301}$ The following stoichiometries were found to lie on the convex hull: $\mathrm{Ge}_{3} \mathrm{H}$ (which was stable with respect to the elements already by $40 \mathrm{GPa}), \mathrm{Ge}_{2} \mathrm{H}, \mathrm{GeH}_{3}, \mathrm{Ge}_{3} \mathrm{H}_{11}$ and $\mathrm{GeH}_{4}$. Various $\mathrm{GeH}_{3}$ phases were found to be superconducting with estimated $T_{c}$ values that exceeded $100 \mathrm{~K}$ at $180 \mathrm{GPa}, \stackrel{302}{ }$ and $80 \mathrm{~K}$ at $300 \mathrm{GPa} \cdot{ }^{300}$ The $T_{c} \mathrm{~s}$ of $C 2 / m-\mathrm{GeH}_{4}$ and $I \overline{4} m 2-\mathrm{Ge}_{3} \mathrm{H}_{11}$ were predicted to be $56-67 \mathrm{~K}$ at $280 \mathrm{GPa}$, and $34-43 \mathrm{~K}$ at $285 \mathrm{GPa}$, respectively, and they were found to decrease at higher pressures. $\underline{301}$

Tin

A number of theoretical studies have examined the high pressure behavior of stannane, $\mathrm{SnH}_{4}$, which is an unstable molecule at atmospheric pressures. Simulated annealing calculations predicted a metallic, $P 6 / \mathrm{mmm}$-symmetry structure as having the lowest enthalpy between 70 $160 \mathrm{GPa} \cdot \stackrel{303}{ }$ Soft phonon modes resulting from Fermi surface nesting were found to give rise to a large EPC and a $T_{c}$ of $80 \mathrm{~K}$ at $120 \mathrm{GPa}$. A couple of years later evolutionary searches uncovered two $\mathrm{SnH}_{4}$ phases with lower enthalpies, an $A m a 2$ structure that was stable between 96-180 GPa and a $P 6_{3} / m m c$ symmetry phase that was preferred above $180 \mathrm{GPa} \cdot{ }^{304}$ Both of these phases contained hexagonal layers of $\mathrm{Sn}$ atoms and $\mathrm{H}_{2}$ units. It was shown that $\mathrm{SnH}_{4}$ becomes thermodynamically stable with respect to decomposition into the elemental species above $96 \mathrm{GPa}$. The $T_{c}$ was estimated as being $15-22 \mathrm{~K}$ for the $A m a 2$ phase at $120 \mathrm{GPa}$, and $52-62 \mathrm{~K}$ for the $P 6_{3} / m m c$ structure at $200 \mathrm{GPa}$. Computations that considered pressures up to $600 \mathrm{GPa}$ predicted that a $C 2 / m$ symmetry $\mathrm{SnH}_{4}$ phase will become stable at $400 \mathrm{GPa} \cdot{ }^{[05]}$ Its $T_{c}$ was estimated as being 64-74 $\mathrm{K}$ at $500 \mathrm{GPa}$, and the EPC was found to arise primarily from the vibrational 
modes of hydrogen. It was shown that at pressures where $\mathrm{SnH}_{4}$ is thermodynamically unstable with respect to the elemental phases, the most stable structures unsurprisingly segregated into single-component slabs or layers, some of which may be kinetically stable. ${ }^{306}$

Two theoretical studies have considered phases with novel hydrogen to tin ratios. Between 250-350 GPa the $I \overline{4} m 2-\mathrm{SnH}_{8}$ structure illustrated in Fig. 13.(c), which contains $\mathrm{H}_{2}$ and slightly bent $\mathrm{H}_{3}$ molecules, was found to lie on the convex hull. ${ }^{295}$ Its $T_{c}$ was calculated to be $63-72 \mathrm{~K}$ at $250 \mathrm{GPa}$, and it increased slightly under pressure. In addition to this phase, a later study also identified $C 2 / m-\mathrm{SnH}_{12}$ and $C 2 / m-\mathrm{SnH}_{14}$, which were predicted to become stable above $250 \mathrm{GPa}$ and $280 \mathrm{GPa}$, respectively. ${ }^{307} \mathrm{~A}$ unique motif of linear $\mathrm{H}_{4}^{-}$units was observed in $\mathrm{SnH}_{12}$, whereas linear $\mathrm{H}_{3}^{-}$moieties were found in $\mathrm{SnH}_{14}$. The $T_{c}$ was estimated as being $81 \mathrm{~K}, 93 \mathrm{~K}$, and $97 \mathrm{~K}$ for $\mathrm{SnH}_{8}$ at $220 \mathrm{GPa}, \mathrm{SnH}_{12}$ at $250 \mathrm{GPa}$ and $\mathrm{SnH}_{14}$ at $300 \mathrm{GPa}$, respectively.

\section{Lead}

The lead tetrahydride analogue of methane, tetrahedral $\mathrm{PbH}_{4}$, is thermodynamically unstable in the gas phase. $\mathrm{PbH}_{4}$ was first synthesized via laser ablation in a solid hydrogen matrix, along with the $\mathrm{Pb}_{2} \mathrm{H}_{2}$ and $\mathrm{H}\left(\mathrm{Pb}_{2} \mathrm{H}_{2}\right) \mathrm{H}$ molecules. ${ }^{308}$ The instability of $\mathrm{PbH}_{4}$ has precluded experimental studies of its solid state structure, and so far the high pressure behavior of this, and other hydrides, of lead have only been investigated theoretically. Calculations showed that solid $\mathrm{PbH}_{4}$ becomes enthalpically favorable with respect to the elemental phases above $132 \mathrm{GPa} \cdot{ }^{309}$ Below $\sim 300 \mathrm{GPa}$ the most stable phase was found to have a three-dimensional lattice, whereas the lowest enthalpy phase above this pressure was distinctly layered. Both phases contained $\mathrm{H}_{2}$ molecules whose intermolecular distances were comparable to those within elemental hydrogen, and these hydrogenic sublattices were found to exhibit liquid-like behavior. They were good metals, with a nearly free electron like DOS. CSP techniques have also been employed to investigate the $\mathrm{PbH}_{4}\left(\mathrm{H}_{2}\right)_{2}$ stoichiometry. ${ }^{296}$ Enthalpically stable structures, which contained $\mathrm{H}_{2}$ molecules that separated the $\mathrm{Pb}$ atoms, were predicted above $133 \mathrm{GPa}$. The $C 2 / m$ symmetry phase illustrated in Fig. 13 (d) was found to be a good metal, with an estimated $T_{c}$ of $107 \mathrm{~K}$ at $230 \mathrm{GPa}$, and the large electron-phonon coupling was primarily due to vibrations associated with the hydrogen atoms.

\section{Group 15: Pnictogen Hydrides}

\section{Nitrogen}

At ambient pressure and temperature gas phase $\mathrm{NH}_{3}$ is the only pnictogen hydride that is thermodynamically stable. Six molecular $\mathrm{NH}_{3}$ phases have been studied experimentally including: a low-temperature ordered phase, ${ }^{310}$ higher temperature rotationally disordered phases II and III, an orthorhombic phase IV, $\stackrel{311312}{ }$ phase V (whose spacegroup is unknown), ${ }^{313}$ and phase VI (which may exhibit symmetric hydrogen bonding). ${ }^{312}$ DFT calculations showed that hydrogen bond symmetrization does not occur in ammonia up to at least $300 \mathrm{GPa}$, but it was pointed out that quantum proton motion may promote symmetrization at lower pressures. ${ }^{314}$ Random searches at $0 \mathrm{~K}$ found the previously reported phase I and phase IV structures. ${ }^{315}$ However, above $90 \mathrm{GPa}$ a previously unknown Pma2 symmetry phase consisting of alternating layers of $\mathrm{NH}_{4}^{+}$and $\mathrm{NH}_{2}^{-}$ions, which had a band gap of $3.6 \mathrm{eV}$ at $100 \mathrm{GPa}$, was found to be the most stable. Experiments provided evidence for the existence of an ionic phase around $150 \mathrm{GPa}$, and further calculations showed that a 
$P c a 2_{1}$ symmetry ionic lattice is more stable than Pma2 above $176 \mathrm{GPa} \cdot \frac{316}{}$ The high pressure behavior of mixtures of $\mathrm{N}_{2}$ and $\mathrm{H}_{2},{ }^{317318}$ as well as $\mathrm{NH}_{3}$ and $\mathrm{H}_{2}{ }^{319}$ has been studied experimentally.

\section{Phosphorus}

Recent resistance measurements on phosphine, $\mathrm{PH}_{3}$, which was liquefied and compressed in a DAC revealed a $T_{c}$ of $30 \mathrm{~K}$ at $83 \mathrm{GPa}$ and $103 \mathrm{~K}$ at $207 \mathrm{GPa} \cdot \frac{320}{}$ The lack of experimental structural information motivated a series of theoretical studies that used CSP to identify several candidate structures for the superconducting phases between 100-200 GPa including PH, $\mathrm{PH}_{2}$, ,21/322 and $\mathrm{PH}_{3} \cdot{ }^{321323}$ Even though the most stable phases identified were found to be unstable with respect to decomposition into the elements under pressure, they were dynamically stable and superconducting. The experimental pressure dependence of the $T_{c}$ agreed most closely with that of the $I 4 / \mathrm{mmm}$ symmetry $\mathrm{PH}_{2}$ structure ${ }^{321}$ illustrated in Fig. 14(a), but it was concluded that the observed superconductivity is likely due to a mixture of metastable phases that form from the decomposition of phosphine under pressure. Migdal-Eliashberg theory has been employed to study the superconducting properties of one of the predicted phases with the $\mathrm{PH}_{3}$ stoichiometry. ${ }^{324}$ Calculations have also been undertaken to investigate the $\mathrm{P} / \mathrm{H}$ phase diagram at $P<100 \mathrm{GPa} \cdot{ }^{325}$ The structure with the most negative $\Delta H_{F}$ at $80 \mathrm{GPa}$ was a non-metallic $\left(\mathrm{PH}_{5}\right)_{2}$ phase whose structure and bonding was analogous to that of diborane. A number of metastable phases that were composed of hydrogen-capped simple cubic like phosphorus layers and mobile molecular $\mathrm{H}_{2}$ layers were found to be superconducting. However, the $T_{c}$ of the two $\mathrm{PH}_{2}$ phases illustrated in Fig. 14(b) and Fig. 14(c) agreed the best with the experimental results. The maximum $T_{c}$ s calculated for $\mathrm{PH}, \mathrm{PH}_{2}$ and $\mathrm{PH}_{3}$ are provided in Fig. 15 .

(a)

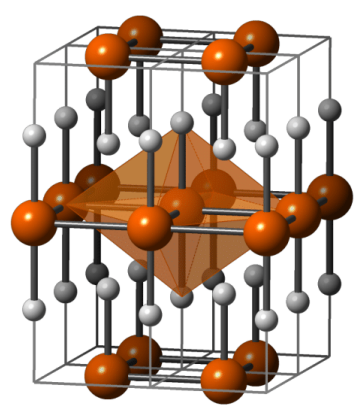

$14 / \mathrm{mmm} \mathrm{PH}_{2}$

$200 \mathrm{GPa}$ (b)

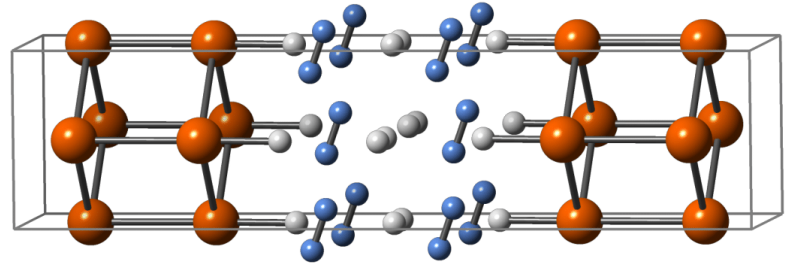

$\mathrm{C} 2 / \mathrm{m} \mathrm{PH}_{2}$

$80 \mathrm{GPa}$

(c)

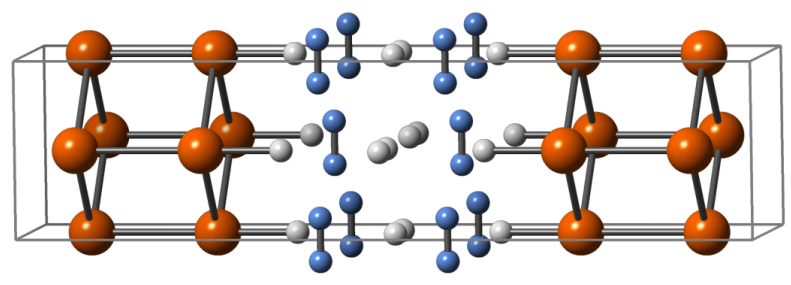

$\mathrm{Cmmm} \mathrm{PH}_{2}$

$80 \mathrm{GPa}$

Figure 14: Theoretically predicted $\mathrm{PH}_{2}$ phases that are likely contributors to the superconductiv-

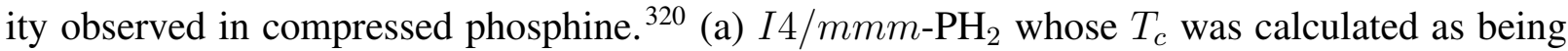
$\sim 70 \mathrm{~K}$ at $200 \mathrm{GPa},{ }^{\sqrt[321 / 322]{ }}$ (b) $C 2 / m-\mathrm{PH}_{2}$ and $C m m m-\mathrm{PH}_{2}$ differ only by a rotation of the $\mathrm{H}_{2}$ molecules colored purple. Their $T_{c}$ was estimated to be $\sim 40 \mathrm{~K}$ at $80 \mathrm{GPa} . \underline{325}$ 


\section{Arsenic, Antimony, Bismuth}

Only a few theoretical studies have investigated the heavier pnictogen polyhydrides, and the highest $T_{c}$ s obtained for each superconducting phase are plotted in Fig. 15. ${ }^{326}, 329 \mathrm{Fu}$ and co-workers systematically explored the hydrogen rich phase diagram of the hydrides of $\mathrm{P}, \mathrm{As}$, and $\mathrm{Sb} \cdot \frac{326}{\mathrm{The}}$ crystalline hydrides of phosphorus were found to be unstable with respect to decomposition into the elements between 100-400 GPa, whereas those of arsenic became stable by $300 \mathrm{GPa}$, and antimony by $200 \mathrm{GPa}$. The predicted stable structures included: $\mathrm{Cmcm}$-AsH, which adopts a threedimensional network with five-coordinate As and $\mathrm{H}$ atoms; $C 2 / c-\mathrm{AsH}_{8}$, which is formed from irregular $\mathrm{AsH}_{16}$ polyhedral motifs connected to one another in a three-dimensional network containing quasi-molecular $\mathrm{H}_{2}$ units with a bond length of 0.8-0.9 $\AA$; Pnma-SbH, which is composed of $\mathrm{Sb}-\mathrm{H}$ chain-like motifs where each $\mathrm{Sb}$ atom is coordinated to three $\mathrm{H}$ atoms; $\mathrm{Pmmn}-\mathrm{SbH}_{3}$, which is comprised of irregular $\mathrm{SbH}_{10}$ and $\mathrm{SbH}_{12}$ polyhedra with quasi-molecular $\mathrm{H}_{2}$ bridges; and $\mathrm{P}_{3} / \mathrm{mmc}_{\mathrm{SbH}}$, which is made up of regular $\mathrm{SbH}_{14}$ octadecahedra that are connected through shared corner $\mathrm{H}$ atoms in a three-dimensional network forming quasi-molecular $\mathrm{H}_{2}$ units. The estimated $T_{c} \mathrm{~s}$ of $\mathrm{AsH}_{8}$ and $\mathrm{SbH}_{4}$ were $\sim 150 \mathrm{~K}$ at $350 \mathrm{GPa}$ and $100 \mathrm{~K}$ at $150 \mathrm{GPa}$, respectively, whereas all other compounds possessed a $T_{c}$ of $\sim 20 \mathrm{~K}$ or lower. The same $P 6_{3} / m m c-\mathrm{SbH}_{4}$ phase was predicted in a prior work. ${ }^{327}$ Abe and Ashcroft computationally studied the $\mathrm{SbH}_{2}$ and $\mathrm{SbH}_{3}$ stoichiometries, and they found that at $170 \mathrm{GPa} P n m a-\mathrm{SbH}_{3}$ was stable and superconducting with a $T_{c}$ of $\sim 68 \mathrm{~K}$ for $\mu^{*}=0.13 . \frac{328}{}$ The hydrides of bismuth were calculated to become stable with respect to the elemental phases above $105 \mathrm{GPa} \cdot{ }^{328329} \mathrm{P}_{3} / \mathrm{mmc} \mathrm{BiH}$ contained monoatomic hydrogen atoms, whereas quasi-molecular $\mathrm{H}_{2}$ units were present within many of the $\mathrm{BiH}_{n}(n=2-6)$ phases. In addition, $C 2 / m-\mathrm{BiH}_{5}$ was also comprised of linear $\mathrm{H}_{3}$ units. ${ }^{[329} T_{c} \mathrm{~s}$ ranging from $20-119 \mathrm{~K}$ have been calculated for these hydrides, and the highest value obtained was for $\mathrm{BiH}_{5}$ at $300 \mathrm{GPa}$.

\section{Group 16: Chalcogen Hydrides}

\section{Oxygen}

The phase diagram of $\mathrm{H}_{2} \mathrm{O}$ has been extensively studied because of its relevance towards life on earth, as well as the interiors of icy extraterrestrial objects. Sixteen crystalline phases of $\mathrm{H}_{2} \mathrm{O}$ have been found experimentally. ${ }^{330}$ Perhaps the most well-known high pressure phase of water is ice $\mathrm{X}$, within which the oxygen atoms are found on a bcc lattice and each hydrogen atom lies midway between two oxygen atoms so that the intra and intermolecular $\mathrm{H}-\mathrm{O}$ distances become

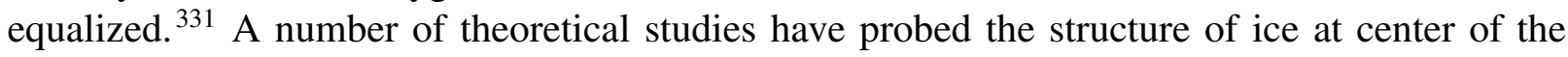
earth pressures and higher. A Pbcm structure was predicted to become stable at $\sim 300 \mathrm{GPa}$. 332 Over a decade later it was shown that it transitions to a $\mathrm{Pbca}$ structure at $0.76 \mathrm{TPa}$, followed by a metallic $C m c m$ phase at $1.55 \mathrm{TPa} \cdot{ }^{333}$ A couple of years later four theoretical studies appeared nearly simultaneously that used different CSP techniques to predict the structures of water at TPa pressures. ${ }^{334}$. 337 The calculations differed not only in the structure prediction methods that were employed, but also in the pressures at which the searches were carried out, and in the sizes of the unit cells considered. As a result, each study proposed a somewhat different phase diagram. One thing that they all had in common, however, was the discovery of insulating phases that were 


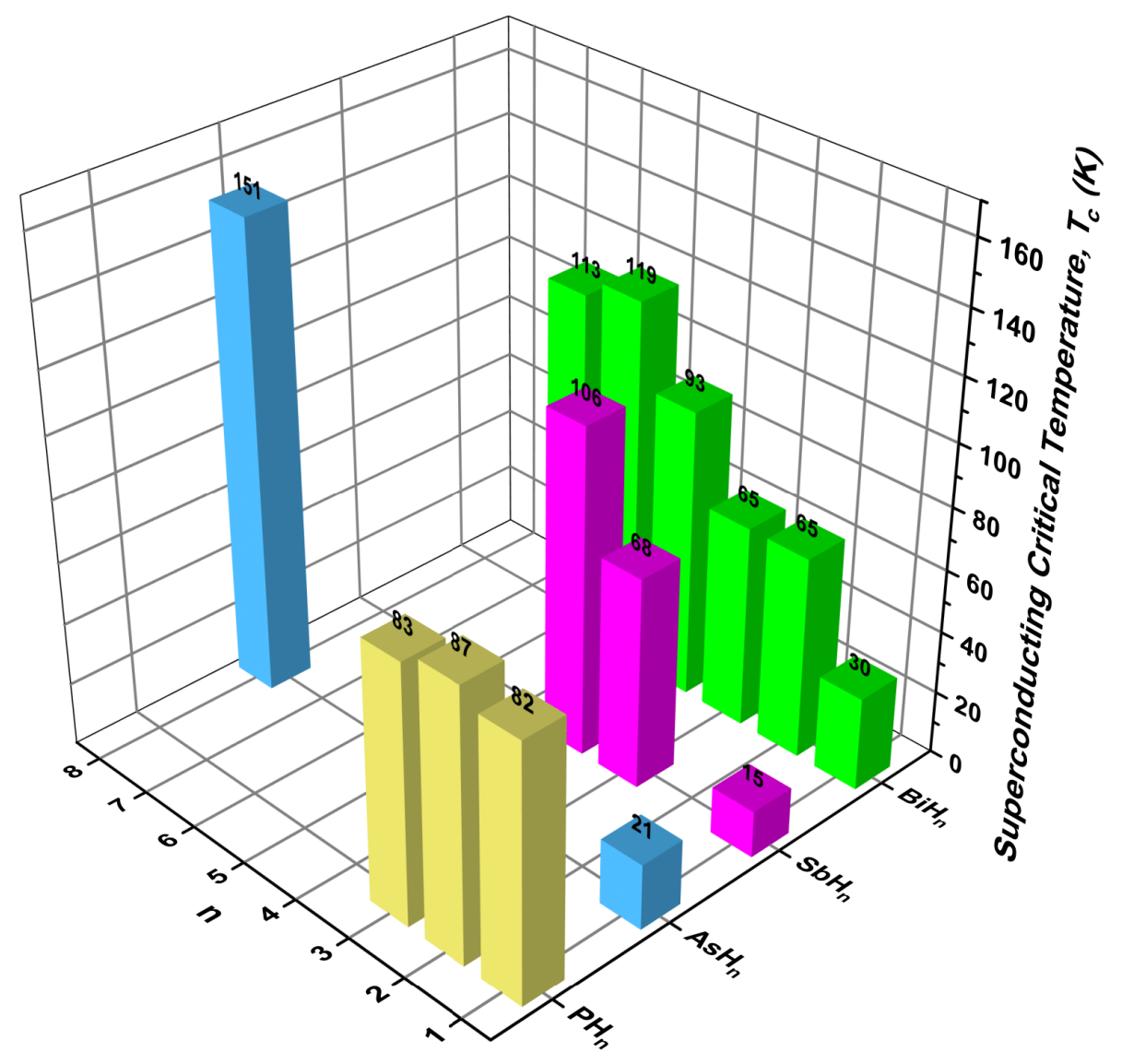

Figure 15: Theoretically predicted $T_{c}$ values for hydrides of group 15 elements, including $\mathrm{PH}_{n}$ (yellow), ${ }^{321+325} \mathrm{AsH}_{n}$ (blue), ${ }^{326} \mathrm{SbH}_{n}$ (pink) ${ }^{326}$ and $\mathrm{BiH}_{n}$ (green), ${ }^{3283329} n=1-8$. The $T_{c} \mathrm{~s}$ provided are the highest ones obtained for the given composition, and may have been calculated at different pressures and using slightly different values of $\mu^{*}$.

more stable than the previously proposed metallic $\mathrm{Cmca}-\mathrm{H}_{2} \mathrm{O}$ phase. Thus, the pressure at which ice is thought to become metallic was shifted to higher values; somewhere between 2-7 TPa.

Because $\mathrm{H}_{2} \mathrm{O}$ and $\mathrm{H}_{2}$ are known to form hydrogen clathrate compounds at high pressures,, 338 evolutionary algorithms have been employed to search for hitherto unknown $\mathrm{H}_{2} \mathrm{O}-\mathrm{H}_{2}$ clathratelike structures up to $100 \mathrm{GPa} \cdot{ }^{339}$ Moreover, CSP methods have found that at planetary pressures non-intuitive reactions and decomposition mechanisms of binary $\mathrm{H} / \mathrm{O}$ systems can occur. For example, above $5 \mathrm{TPa} \mathrm{H}_{2} \mathrm{O}$ is predicted to decompose into $\mathrm{H}_{2} \mathrm{O}_{2}$ and hydrogen rich phases, ${ }_{340}$ and at 1.4 TPa an $\mathrm{H}_{4} \mathrm{O}$ structure is preferred over elemental water and hydrogen. 341

\section{Sulfur}

The discovery of high temperature superconductivity in the high pressure hydrogen/sulfur phase diagram is a striking example of how a feedback loop between experiment and theory can lead to the synthesis of remarkable materials. A recent mini-review provides an excellent synopsis of the work carried out on this system to date. ${ }^{50}$ Hydrogen sulfide, $\mathrm{H}_{2} \mathrm{~S}$, has been theoretically 
and experimentally studied extensively under pressure. ${ }^{342,357}$ For example, Ab Initio molecular dynamics computations ${ }^{353}-355$ and CSP techniques ${ }^{\sqrt{356}}$ have been employed to propose structural candidates for various phases. Li et al. showed that $\mathrm{H}_{2} \mathrm{~S}$ is thermodynamically stable with respect to the elemental phases up to $200 \mathrm{GPa}$, and proposed candidate structures for the non-metallic phases IV and V. ${ }^{\sqrt[356]{6}}$ Moreover, they calculated a $T_{c}$ of $80 \mathrm{~K}$ at $160 \mathrm{GPa}$ for the $C m c a$ phase illustrated in Fig. 16.(a). 356

Strobel and co-workers carried out experiments that showed compound formation between $\mathrm{H}_{2} \mathrm{~S}$ and $\mathrm{H}_{2}$ at $3.5 \mathrm{GPa}$, and evidence of a clathrate-like structure by $17 \mathrm{GPa} \cdot{ }^{358}$ This work inspired a computational study by Duan and co-workers on the high pressure behavior of systems with the $\left(\mathrm{H}_{2} \mathrm{~S}\right)_{2} \mathrm{H}_{2}$ (or $\mathrm{H}_{3} \mathrm{~S}$ ) stoichiometry. ${ }^{359}$ DFT calculations predicted that metallic $R 3 \mathrm{~m}$ and $\mathrm{Im} \overline{3} \mathrm{~m}$ symmetry $\mathrm{H}_{3} \mathrm{~S}$ phases became preferred above 111 and $180 \mathrm{GPa}$, respectively. The EPC of both structures was found to be particularly high, and the $T_{c}$ was estimated as being $155-166 \mathrm{~K}$ at $130 \mathrm{GPa}$ and $191-204 \mathrm{~K}$ at $200 \mathrm{GPa}$. The cubic $\operatorname{Im} \overline{3} m$ structure is illustrated in Fig. 16.(b).

The theoretical work of $\mathrm{Li}$ and co-workers,, 356 on the other hand, inspired Drozdov et al. to study the superconducting behavior of $\mathrm{H}_{2} \mathrm{~S}$ under pressure. ${ }^{44}$ The measured $T_{c} \mathrm{~s}$ for samples prepared at $T \leq 100 \mathrm{~K}$ were in good agreement with the values computed for $\mathrm{H}_{2} \mathrm{~S}$. ${ }^{[56}$ The much higher $T_{c}$ observed for the sample prepared above room temperature was consistent with the predictions for $\mathrm{H}_{3} \mathrm{~S}$ made by Duan's group, ${ }^{359}$ leading to the suggestion that at these conditions the hydrogen sulfide decomposed into elemental sulfur and $\mathrm{H}_{3} \mathrm{~S}$, and the $\mathrm{H}_{3} \mathrm{~S}$ phase gave rise to the remarkable superconductivity. XRD has shown that the sulfur positions in the superconducting phase are consistent with the theoretically predicted $I m \overline{3} m$ and $R 3 m-\mathrm{H}_{3} \mathrm{~S}$ structures. $\underline{360}$ The Meissner effect confirmed the record breaking $T_{c}, \frac{}{361}$ and a recent optical reflectivity study suggested that this material is a conventional superconductor where the superconductivity is due to electron-phonon interaction. ${ }^{\sqrt[362]{2}}$ The direct synthesis of $\mathrm{H}_{3} \mathrm{~S}$ starting from $\mathrm{H}_{2}$ and $\mathrm{S}$ followed by characterization via XRD and Raman spectroscopy led to the conclusion that the $I m \overline{3} m-\mathrm{H}_{3} \mathrm{~S}$ phase forms above $140 \mathrm{GPa} \cdot{ }^{363}$ Another experimental study, however, provided evidence for the synthesis of a $C c c m-\mathrm{H}_{3} \mathrm{~S}$ phase up to $160 \mathrm{GPa}$, but not for the proposed superconducting $\operatorname{Im} \overline{3} \mathrm{~m}$ and $R 3 m$ symmetry phases. $\frac{364}{36}$

A plethora of theoretical calculations have been carried out to identify the stoichiometry and structure of the superconducting phase, analyze the factors contributing to $T_{c}$, and investigate the effect of anharmonicity, the isotope effect, and the quantum nature of the proton. $324 \mid 357 / 367-\sqrt[383]{3} \mathrm{~A}$ majority of these focused on the $I m \overline{3} m$ symmetry $\mathrm{H}_{3} \mathrm{~S}$ phase. However, a few recent studies have questioned if this phase is responsible for the observed superconductivity. For example, it was shown that the decomposition of $\operatorname{Im} \overline{3} m-\mathrm{H}_{3} \mathrm{~S}$ into $\mathrm{H}_{2}$ plus an $\mathrm{SH}_{2}$ phase, which can be thought of as an $\left(\mathrm{SH}^{-}\right)\left(\mathrm{H}_{3} \mathrm{~S}^{+}\right)$perovskite structure, is favorable. ${ }^{[384}$ Ab initio molecular dynamics (AIMD) simulations at $200 \mathrm{GPa}$ and $200 \mathrm{~K}$ revealed that this perovskite structure can segregate into cubic and tetragonal regions that form a modulated structure whose diffraction pattern matches well with the one obtained experimentally. ${ }^{[385}$ Another study proposed that an infinite number of metastable long-period modulated crystals formed from the intergrowth of $\mathrm{H}_{2} \mathrm{~S}$ and $\mathrm{H}_{3} \mathrm{~S}$ with a composition of $\mathrm{H}_{x} \mathrm{~S}_{1-x}$ with $2 / 3<x<3 / 4$ (so-called "Magnéli" Phases) could account for the experimentally observed dependence of the $T_{c}$ versus pressure. ${ }^{386}$ Interestingly, the modulated structures predicted via AIMD resemble the Magnéli phases.

In addition, CSP searches have shown that the $\mathrm{H}_{2} \mathrm{~S}_{3}, \mathrm{H}_{3} \mathrm{~S}_{2}, \mathrm{HS}_{2}, \mathrm{H}_{4} \mathrm{~S}_{3},{ }^{365} \mathrm{H}_{5} \mathrm{~S}_{8}, \mathrm{H}_{3} \mathrm{~S}_{5}{ }^{378}$ and $\mathrm{H}_{5} \mathrm{~S}_{2}{ }^{366}$ stoichiometries could potentially form under pressure. The superconducting Pnma- 
(a)

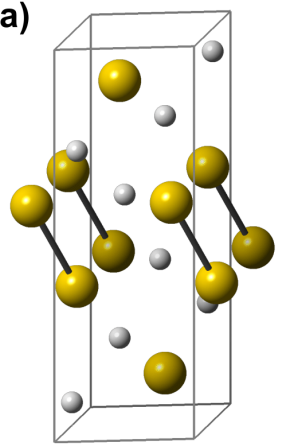

Cmca $\mathrm{H}_{2} \mathrm{~S}$ (b)

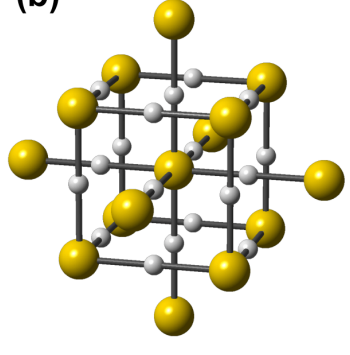

Im $\overline{3} m \mathrm{H}_{3} \mathrm{~S}$ (c)

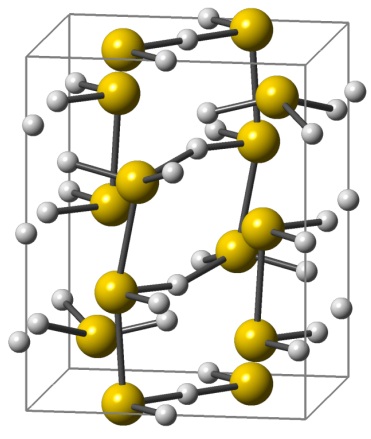

Pnma $\mathrm{H}_{4} \mathrm{~S}_{3}$ (d)

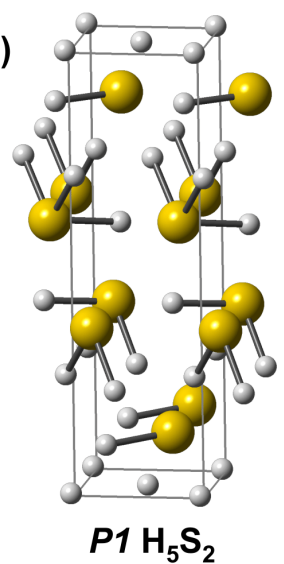

Figure 16: Structures of superconducting phases predicted for compounds containing hydrogen

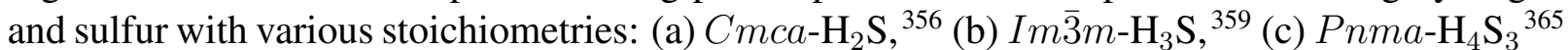
and (d) $P 1-\mathrm{H}_{5} \mathrm{~S}_{2} \cdot \frac{366}{}$

$\mathrm{H}_{4} \mathrm{~S}_{3}$ and $P 1-\mathrm{H}_{5} \mathrm{~S}_{2}$ structures are illustrated in Fig. 16(c) and Fig. 16(d), respectively. Moreover, a recent study concluded that $\mathrm{H}_{2} \mathrm{~S}$ can be kinetically protected up to very high pressures and only this phase can account for the superconductivity observed by Drozdov and co-workers in the samples prepared at low temperatures. $\underline{365}$

Theoretical work assuming the $I m \overline{3} m-\mathrm{H}_{3} \mathrm{~S}$ structure showed that the $T_{c}$ could further be increased by substitution of sulfur with more electronegative elements. ${ }^{387}$ Another study predicted a maximum superconducting critical temperature of $280 \mathrm{~K}$ at $250 \mathrm{GPa}$ for $\mathrm{H}_{3} \mathrm{~S}_{0.925} \mathrm{P}_{0.075} \cdot{ }^{388}$ High temperature superconductivity has also been proposed for other compressed sulfides including $\mathrm{Li}_{2} \mathrm{~S}, \mathrm{Na}_{2} \mathrm{~S}$ and $\mathrm{K}_{2} \mathrm{~S}$

\section{Selenium}

Following the discovery of high $T_{c}$ superconductivity in condensed sulfur hydride, the isoelectronic selenium analog was investigated for its potential superconductivity under high pressure. At $200 \mathrm{GPa}$ and $300 \mathrm{GPa} C 2 / \mathrm{m}$ - $\mathrm{HSe}_{2}$ and $\mathrm{P} 4 / \mathrm{nmm}$-HSe were found to be the lowest points on the convex hull, respectively. $\frac{390}{} \mathrm{An} I m \overline{3} m-\mathrm{H}_{3}$ Se structure that was isostructural to the proposed superconducting $\mathrm{H}_{3} \mathrm{~S}$ phase also lay on the convex hull at both of these pressures. The $T_{c}$ of $\mathrm{H}_{3} \mathrm{Se}$ and $\mathrm{HSe}$ were estimated as being in the range of $100 \mathrm{~K}$ and $40 \mathrm{~K}$, respectively. Another study that was published at about the same time also predicted the high pressure stability of the $\mathrm{Im} \overline{3} \mathrm{~m}$ $\mathrm{H}_{3}$ Se phase, and SCDFT (density functional theory for superconductors) calculations yielded a $T_{c}$ of $130 \mathrm{~K}$ at $200 \mathrm{GPa} \cdot{ }^{367}$ Its decreased $T_{c}$ as compared with the sulfur containing compound is a result of a smaller EPC, which likely originates from the better screening of the hydrogen vibrations by the larger ionic size of selenium.

In a recent study $\mathrm{H}_{2}$ Se was synthesized from the elemental phases at $0.4 \mathrm{GPa}$ and $473 \mathrm{~K} .391$ At $12 \mathrm{GPa}$ a transition to a structure resembling phase IV of $\mathrm{H}_{2} \mathrm{~S}$ was found to occur. Moreover, a host-guest $I 4 / m c m-\left(\mathrm{H}_{2} \mathrm{Se}\right)_{2} \mathrm{H}_{2}$ structure, which was analogous to the one observed in $\left(\mathrm{H}_{2} \mathrm{~S}\right)_{2} \mathrm{H}_{2}$, formed above $4.2 \mathrm{GPa}$. Both of these hydrides of selenium decomposed above $24 \mathrm{GPa}$ 
at $300 \mathrm{~K} \cdot{ }^{392}$ In addition to $\mathrm{H}_{2} \mathrm{Se}$, a recent study synthesized a $\mathrm{Cccm}-\mathrm{H}_{3}$ Se phase at $4.6 \mathrm{GPa}$ and $300 \mathrm{~K}$. At $170 \mathrm{~K} \mathrm{Cmcm}-\mathrm{H}_{3} \mathrm{Se}$ was found to persist up to $39.5 \mathrm{GPa}$. Raman measurements and visual observations suggested that metalization occurred above $23 \mathrm{GPa}$.

\section{Tellurium}

Computational studies of structures with the $\mathrm{H}_{x} \mathrm{Te}_{y}(x=1-8, y=1-3)$ stoichiometries have been carried out up to $300 \mathrm{GPa}$. CSP techniques found that $P 6 / m m m-\mathrm{H}_{4} \mathrm{Te}, C 2 / m-\mathrm{H}_{5} \mathrm{Te}_{2}$ and $\mathrm{P}_{3} / \mathrm{mmc}$-HTe were stable with respect to the elements by $200 \mathrm{GPa} \cdot{ }^{393} \mathrm{In}$ addition to these a $C 2 / c-\mathrm{HTe}_{3}$ phase was present on the $300 \mathrm{GPa}$ convex hull. Quasi-molecular $\mathrm{H}_{2}$ units were found within $\mathrm{H}_{4} \mathrm{Te}$ and $\mathrm{H}_{5}$ Te. The latter phase also possessed linear $\mathrm{H}_{3}$ motifs. The largest $T_{c}$ calculated was $104 \mathrm{~K}$ for $\mathrm{H}_{4} \mathrm{Te}$ at $170 \mathrm{GPa}$. The estimated $T_{c}$ for $\mathrm{H}_{5} \mathrm{Te}$ was $58 \mathrm{~K}$ at $200 \mathrm{GPa}$. It was speculated that the structures and stoichiometries of the stable tellurium hydrides differed from those of sulfur and selenium because tellurium has a larger atomic core and smaller electronegativity as compared to its lighter brethren. The main contributions to the $\mathrm{EPC}$ for $\mathrm{H}_{4} \mathrm{Te}$ and $\mathrm{H}_{5} \mathrm{Te}$ arose from the intermediate-frequency hydrogen-based wagging and bending modes, as opposed to the higher frequency $\mathrm{H}$-stretching modes that were found to be so important in hydrides containing sulfur and selenium.

\section{Polonium}

CSP techniques have been employed to find the most stable structures with the $\mathrm{PoH}_{n}, n=1-6$, stoichiometries up to $300 \mathrm{GPa} \cdot{ }^{394}$ The first hydride to become stable with respect to the elements was $\mathrm{PoH}_{2}$ in the $\mathrm{Cmcm}$ spacegroup at $100 \mathrm{GPa}$. At higher pressures the following stable phases were identified: $P 6_{3} / m m c-\mathrm{PoH}, \mathrm{Pnma}-\mathrm{PoH}_{2}, C 2 / c-\mathrm{PoH}_{4}$ and $C 2 / m-\mathrm{PoH}_{6}$. All of these phases were good metals, and with the exception of $\mathrm{PoH}$ they all contained $\mathrm{H}_{2}$ units. Whereas the $T_{c}$ of $\mathrm{PoH}_{4}$ was estimated as being $41-47 \mathrm{~K}$ at $200 \mathrm{GPa}$, the $T_{c}$ of all of the other phases was $<5 \mathrm{~K}$.

\section{Group 17: Halogen Hydrides}

\section{Fluorine, Chlorine, Bromine}

The isomorphic low temperature phases of $\mathrm{HF}, \mathrm{HCl}$ and $\mathrm{HBr}$ contain planar zigzag chains of hydrogen-bonded molecules held together by vdW forces. At atmospheric pressures HF crystallizes in a $C m c 2_{1}$ structure with four formula units in the cell. ${ }^{395}$ Because the hydrogen bonds in the heavier halogen hydrides are weaker, they assume orientationally disordered molecular phases at high temperatures. At room temperature $\mathrm{HCl}$ and $\mathrm{HBr}$ adopt a structure isomorphic to $C m c 2_{1}-\mathrm{HF} .{ }^{3961397} \mathrm{HF}(6 \mathrm{GPa}),{ }^{398} \mathrm{HBr}(32-39 \mathrm{GPa})^{\sqrt[399]{3}}$ and $\mathrm{HCl}(51 \mathrm{GPa})^{400}$ undergo a transformation to a $\mathrm{Cmcm}$ phase wherein all of the $\mathrm{H}-\mathrm{X}$ bonds are symmetric at the pressures given in the parentheses. Whereas the symmetric $\mathrm{HCl}$ phase is stable, the $\mathrm{HBr}$ phase is not $\frac{401}{4}$ and a decomposition reaction that yields $\mathrm{Br}_{2}$ molecules occurs.

Second order Møller-Plesset perturbation theory (MP2) calculations have been performed on solid HF and DF up to $20 \mathrm{GPa} .{ }^{402}$ Moreover, DFT based CSP techniques have been used to study the structural evolution of $\mathrm{HF}, \mathrm{HCl}$ and $\mathrm{HBr}$ up to $200 \mathrm{GPa} \cdot{ }^{403}$ The known $C m c 2_{1}$ and $\mathrm{Cmcm}$ phases were found, and it was predicted that HF would transform to a Pnma phase at $143 \mathrm{GPa}$, whereas $\mathrm{HCl}$ and $\mathrm{HBr}$ were found to assume a $P \overline{1}$ structure above $108 \mathrm{GPa}$ and $59 \mathrm{GPa}$, 
respectively. These phases all contained symmetric $\mathrm{H}-\mathrm{X}$ bonds. Whereas $\mathrm{HF}$ was found to be a large band gap insulator at the highest pressures studied, $\mathrm{HCl}$ and $\mathrm{HBr}$ were semi-metals. A study that followed soft phonon modes calculated with DFT predicted that a $\mathrm{Cmcm} \rightarrow P 2_{1} / \mathrm{m}$ phase transition would occur at 134-196 GPa for $\mathrm{HBr}$ and above $233 \mathrm{GPa}$ for $\mathrm{HCl}$. ${ }^{404}$ The $T_{c}$ was estimated to be $27-34 \mathrm{~K}$ for $\mathrm{HBr}$ at $160 \mathrm{GPa}$ and $9-14 \mathrm{~K}$ for $\mathrm{HCl}$ at $280 \mathrm{GPa}$. Five years later CSP techniques were yet again employed to search for the most stable phases of $\mathrm{HBr} 405 / 406$ and $\mathrm{HCl}^{405}$ under pressure. Both studies showed that based on the enthalpy alone, $\mathrm{HBr}$ was not likely to decompose into the elemental phases under pressure. An $I \overline{4} 2 d-\mathrm{HBr} \frac{\sqrt[406]{ }}{2}$ phase that was nearly isoenthalpic with $P \overline{1}-\mathrm{HBr}$ up to $125 \mathrm{GPa}$ was discovered. Above 120-125 GPa both studies predicted that a $C 2 / m$ symmetry $\mathrm{HBr}$ phase ${ }^{405 / 406}$ is the most stable. The $C 2 / m$ symmetry $\mathrm{HBr}$ phases found in the two studies are different and their estimated $T_{c}$ values are: $25 \mathrm{~K}$ at $150 \mathrm{GPa}{ }^{406}$ and $<1 \mathrm{~K}$ at $120 \mathrm{GPa} \cdot{ }^{405} \mathrm{~A} C 2 / m$ symmetry structure was also found to be the most stable $\mathrm{HCl}$ phase above $250 \mathrm{GPa}$, and at this pressure its $T_{c}$ was estimated as being $20 \mathrm{~K} .{ }^{405}$

(a)

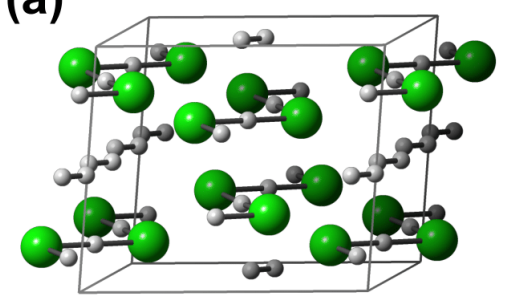

$\mathrm{C} 2 / \mathrm{CH}_{2} \mathrm{Cl}$

(c)

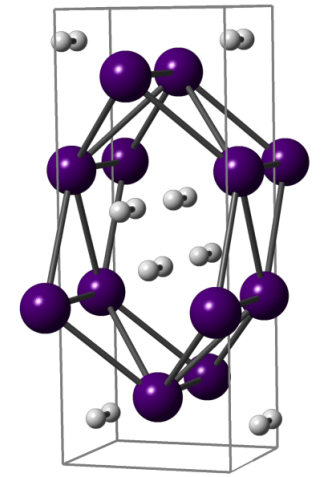

$\mathrm{Cmcm} \mathrm{H}_{2} \mathrm{I}$ (b)

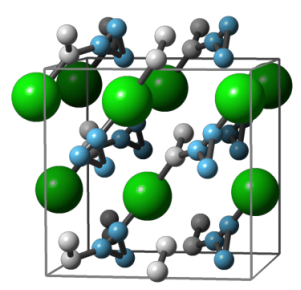

$\mathrm{Cc} \mathrm{H}_{5} \mathrm{Cl}$

(d)

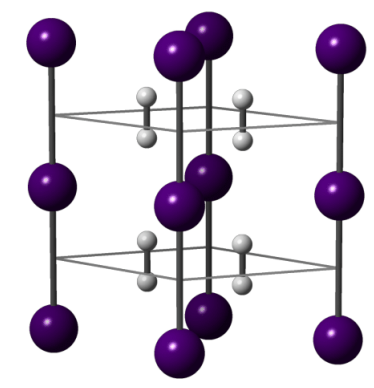

$P 6 / \mathrm{mmm} \mathrm{H}_{4} \mathrm{I}$

Figure 17: Halogen polyhydrides that are predicted to be stable under pressure. (a) $C 2 / c-\mathrm{H}_{2} \mathrm{Cl}$ contains hydrogen atoms and $\mathrm{H}_{2}$ units, ${ }_{407}^{4}$ (b) $\mathrm{Cc}-\mathrm{H}_{5} \mathrm{Cl}$ contains triangular $\mathrm{H}_{3}^{+}$units (colored in light blue) and $\mathrm{H}_{2}$ molecules, ${ }^{407}$ (c) $\mathrm{Cmcm}-\mathrm{H}_{2} \mathrm{I}$ and (d) $\mathrm{P6} / \mathrm{mmm}-\mathrm{H}_{4} \mathrm{I}$ contain $\mathrm{H}_{2}$ units. $\frac{4084409}{4}$

More recently, CSP has been employed to study polyhydrides of the halogens under pressure. $\mathrm{H}_{2} \mathrm{Cl}$ and $\mathrm{H}_{5} \mathrm{Cl}$ were predicted to become stable with respect to decomposition into $\mathrm{H}_{2}$ 
and $\mathrm{HCl}$ between 60-300 GPa, and they lay on the 100, 200 and $300 \mathrm{GPa}$ convex hulls. ${ }^{407}$ The $\mathrm{C} 2 / \mathrm{c}-\mathrm{H}_{2} \mathrm{Cl}$ phase illustrated in Fig. 17(a) contained one-dimensional $\mathrm{HCl}$ chains interposed with $\mathrm{H}_{2}$ molecules. As shown in Fig. 17(b), the three-center-two-electron $\mathrm{H}_{3}^{+}$motif, which was first found in interstellar space, was present within $\mathrm{Cc}-\mathrm{H}_{5} \mathrm{Cl}$. By $300 \mathrm{GPa}$ it approached the ideal equilateral triangle configuration. In addition to these phases a later CSP study found that $\mathrm{H}_{3} \mathrm{Cl}$ lies on the 20,50, and $100 \mathrm{GPa}$ convex hulls, and $\mathrm{H}_{7} \mathrm{Cl}$ comprises the $100 \mathrm{GPa}$ hull. $\frac{410}{}$ None of the aforementioned phases were superconducting. Two years later CSP added one more stoichiometry, $\mathrm{H}_{4} \mathrm{Cl}_{7}$, to the 100-400 GPa convex hulls. ${ }^{411}$ Moreover, the ZPE was found to stabilize a few $\mathrm{H}_{n} \mathrm{Cl}(n=2,3,5)$ phases that had not been predicted previously. One example was a metallic $R \overline{3} m-\mathrm{H}_{2} \mathrm{Cl}$ phase that was superconducting below $44 \mathrm{~K}$ at $400 \mathrm{GPa}$.

A CSP study showed that the only hydride of fluorine that lies on the convex hull under pressure is HF. ${ }^{410}$ Finally, $\mathrm{H}_{n} \mathrm{Br}$ phases with $n=2-5,7$ were shown to be thermodynamically stable above $40 \mathrm{GPa} .{ }^{412}$ At $240 \mathrm{GPa} C m c m-\mathrm{H}_{2} \mathrm{Br}$. and $P 6_{3} / m m c-\mathrm{H}_{4} \mathrm{Br}$ were predicted to be superconducting below $12 \mathrm{~K}$ and $2.4 \mathrm{~K}$, respectively.

\section{Iodine}

The behavior of the monohydride of iodine differs from its lighter brethren. At atmospheric conditions HI assumes a planar distorted hydrogen-bonded diamond lattice, but its detailed structure is unknown. ${ }^{397}$ Pressure induces an insulator to metal transition in HI below $50 \mathrm{GPa}, \frac{413}{4}$ but the impurities and side-products observed above $70 \mathrm{GPa}$ are thought to be indicative of decomposition. ${ }^{414}$ Indeed, CSP calculations showed that already at $1 \mathrm{~atm} \mathrm{HI}$ is metastable with respect to the elemental phases. ${ }^{408}$ This same study found that an insulating $P \overline{1}-\mathrm{H}_{5} \mathrm{I}$ phase, which is comprised of $(\mathrm{HI})^{\delta+}$ and $\mathrm{H}_{2}^{\delta-}$ molecules, is stable between 30-90 GPa. Moreover, the metallic $C m c m-\mathrm{H}_{2} \mathrm{I}$

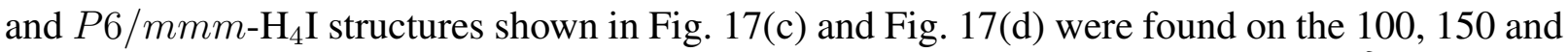
$200 \mathrm{GPa}$ convex hulls. These phases contained monoatomic iodine lattices and $\mathrm{H}_{2}^{\delta-}$ units. At $100 \mathrm{GPa}$ they were estimated to become superconducting below $7.8 \mathrm{~K}$ and $17.5 \mathrm{~K}$, respectively. These two phases were also found by Duan and coworkers who in addition predicted a $R \overline{3} \mathrm{~m}$ symmetry $\mathrm{H}_{2}$ I phase wherein the $\mathrm{H}_{2}$ units had dissociated that had a $T_{c}$ of $33 \mathrm{~K}$ at $240 \mathrm{GPa} .{ }^{409}$

Recently, a novel $\mathrm{HI}\left(\mathrm{H}_{2}\right)_{13}$ molecular compound, which was stable between 9 and $130 \mathrm{GPa}$, was synthesized using $\mathrm{I}_{2}$ and $\mathrm{H}_{2}$ as starting materials. ${ }^{415}$ AIMD simulations showed that this phase adopts $F m \overline{3} c$ symmetry. Compression of $\mathrm{HI}$ and $\mathrm{H}_{2}$ led to the formation of an $I 4 / \mathrm{mcm}$ symmetry $\mathrm{H}_{2}(\mathrm{HI})_{2}$ phase that was stable between 3.5 to $12.5 \mathrm{GPa}$ instead. Superconductivity was not found in these phases, however it was noted that the $\mathrm{H}_{2}$ content of $\mathrm{HI}\left(\mathrm{H}_{2}\right)_{13}$ is high; $93 \mathrm{~mol} \%$, and 17.7 weight $\%$.

\section{Astatine}

To the best of our knowledge, the hydrides of astatine have not yet been studied theoretically nor experimentally.

\section{Group 18: Aerogen Hydrides}

\section{Helium, Neon}

Because the Jovian planets are primarily composed of helium and hydrogen, the astrophysical 
community has been intensely interested in the behavior of these elements upon mixing at high pressures. The calculated Gibbs free energies of mixing of $\mathrm{He}$ and $\mathrm{Ne}$ over a wide range of density, temperature and composition have led to the conclusion that at conditions resembling those of the interior of Saturn the two elements are likely to separate. ${ }^{416 / 417}$ Such phase segregation has been observed in $\mathrm{H}_{2}-\mathrm{He}$ mixtures in a DAC up to $8 \mathrm{GPa}$ at $300 \mathrm{~K} .{ }^{418}$ Moreover, experiments have shown that when the $\mathrm{Ne}$ concentration is between $0.25-99.5 \%, \mathrm{Ne}$ and $\mathrm{H}_{2}$ are immiscible. ${ }^{419}$ To the best of our knowledge no solid binary compounds of these two elements have been made under pressure (but a binary $\mathrm{Ne}\left(\mathrm{He}_{2}\right)_{2}$ compound has been experimentally observed and theoretically studied ${ }^{420[421}$ ).

\section{Argon, Krypton, Xenon}

An interesting, yet intuitive, trend emerges for the heavier noble gas hydrides under pressure; namely that the maximum hydrogen content in the binary compounds is proportional to the size of the aerogen atom. Specifically, the following stoichiometries, illustrated in Fig. 18, have been identified in experiment: $\operatorname{Ar}\left(\mathrm{H}_{2}\right)_{2},{ }^{422] 423} \mathrm{Kr}\left(\mathrm{H}_{2}\right)_{4}, \stackrel{424}{{ }^{424}}$ and $\mathrm{Xe}\left(\mathrm{H}_{2}\right)_{8}$.

In the mid 1990s experiments showed evidence for the formation of an $\operatorname{Ar}\left(\mathrm{H}_{2}\right)_{2}$ compound at $4.3 \mathrm{GPa}$ that adopted a structure isomorphous with the $\mathrm{MgZn}_{2}$ Laves phase. ${ }^{422}$ Raman measurements indicated that this structure was stable up to $175 \mathrm{GPa}$, at which point the $\mathrm{H}_{2}$ molecules started to dissociate and undergo metalization. IR experiments up to $220 \mathrm{GPa}$ questioned this conclusion, since they showed the persistence of molecular hydrogen within this phase.$^{6}$ A number of theoretical studies have been carried out in an attempt to explain this discrepancy. Tight-binding calculations predicted that band gap closure would occur above $400 \mathrm{GPa}$ in $\operatorname{Ar}\left(\mathrm{H}_{2}\right)_{2} \cdot{ }^{427}$ On the other hand, AIMD simulations suggested that an $\mathrm{MgZn}_{2} \rightarrow \mathrm{AlB}_{2}$ structural transition would occur around $250 \mathrm{GPa}$, with concomitant metalization. ${ }^{428}$ More recent GGA calculations found that band gap closure occurs at $420 \mathrm{GPa}$ within the $\mathrm{AlB}_{2}$ structure. ${ }^{429}$ Another AIMD simulation concluded that the structures $\operatorname{Ar}\left(\mathrm{H}_{2}\right)_{2}$ adopts are temperature dependent, with the $\mathrm{MgCu}_{2}$ phase being more stable than the $\mathrm{MgZn}_{2}$ and $\mathrm{AlB}_{2}$ phases below $215 \mathrm{GPa}$ at $0 \mathrm{~K} .{ }^{430}$ Moreover, across a broad pressure range the $\mathrm{MgZn}_{2}$ geometry was found to be favored above $\sim 60-100 \mathrm{~K}$. CSP techniques have also been applied to find the most stable structures up to $300 \mathrm{GPa} \cdot{ }^{431}$ Below $66 \mathrm{GPa}$ the $\mathrm{MgCu}_{2}$ structure was found to be only slightly more stable than the $\mathrm{MgZn}_{2}$ alternative. However, a hitherto unconsidered $\mathrm{CeCu}_{2}$ structure was clearly the lowest enthalpy candidate above $66 \mathrm{GPa}$. Band gap closure in this phase occurred at a pressure higher than that necessary to metallize hydrogen at the same level of theory. This was explained by noting that at a given pressure the intermolecular $\mathrm{H}-\mathrm{H}$ distances in $\operatorname{Ar}\left(\mathrm{H}_{2}\right)_{2}$ are larger than those in pure hydrogen because of the presence of the noble gas, thereby decreasing the orbital overlap. This issue was finally resolved in a 2017 study that employed synchrotron XRD, as well as Raman and optical spectroscopy. ${ }^{423}$ It was shown that $\mathrm{Ar}\left(\mathrm{H}_{2}\right)_{2}$ retains the $\mathrm{MgZn}_{2}$ structure with molecular $\mathrm{H}_{2}$ units up to $358 \mathrm{GPa}$, at which point it had a $2 \mathrm{eV}$ band gap.

Only one study has been carried out on the hydrides of krypton. $\mathrm{A} \mathrm{Kr}\left(\mathrm{H}_{2}\right)_{4}$ phase was synthesized above $5.3 \mathrm{GPa} \cdot{ }^{424}$ Its $\mathrm{Kr}$ sublattice possessed the $F m \overline{3} m$ spacegroup, with rotationally disordered $\mathrm{H}_{2}$ molecules occupying the interstitial sites up to $50 \mathrm{GPa}$.

Experiments in a DAC up to $255 \mathrm{GPa}$ showed crystallographic and spectroscopic evidence

for the formation of $\mathrm{Xe}-\mathrm{H}_{2}$ compounds. ${ }^{425}$ At $4.8 \mathrm{GPa}$ a vdW compound that can be viewed as a superstructure based on the hcp lattice of solid hydrogen modulated by layers containing xenon 


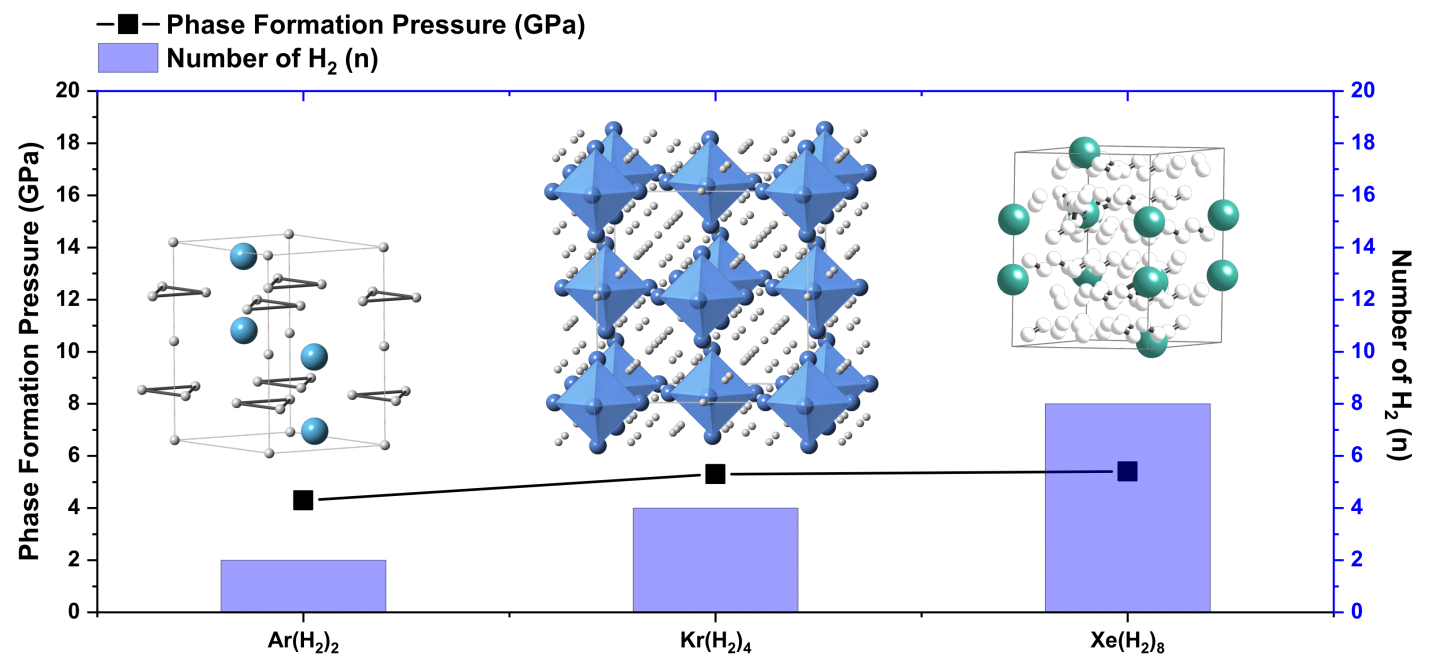

Figure 18: Supercells of the vdW compounds: $\operatorname{Ar}\left(\mathrm{H}_{2}\right)_{2},{ }^{422 \mid 423} \operatorname{Kr}\left(\mathrm{H}_{2}\right)_{4},{ }^{424}$ and $\mathrm{Xe}\left(\mathrm{H}_{2}\right)_{8} .{ }^{425 \mid 426}$ The black line denotes the pressures at which these phases were synthesized. The purple bars correspond to the number of $\mathrm{H}_{2}$ molecules in the phase. The larger the aerogen, the larger the number of $\mathrm{H}_{2}$ molecules that can be accommodated.

dimers was formed. Its stoichiometry was estimated as being $\mathrm{Xe}\left(\mathrm{H}_{2}\right)_{7}$ below, and $\mathrm{Xe}\left(\mathrm{H}_{2}\right)_{8}$ above $5.4 \mathrm{GPa}$. Increasing the pressure increased the chemical interaction within and between the xenon dimers, and it also weakened the $\mathrm{H}-\mathrm{H}$ bond. This $R 3$ symmetry phase remained semiconducting to the highest pressures studied. Because only the positions of the Xe atoms could be determined experimentally, CSP techniques were employed to find the preferred positions of the hydrogen atoms in $\mathrm{Xe}\left(\mathrm{H}_{2}\right)_{7}$ and $\mathrm{Xe}\left(\mathrm{H}_{2}\right)_{8} \cdot{ }^{432} \mathrm{~A}$ Bader charge analysis showed that at $263 \mathrm{GPa}$ the $\mathrm{Xe}$ atoms lost on average $0.5 e$ to the hydrogen atoms. $\mathrm{GW}$ calculations predicted that $\mathrm{Xe}\left(\mathrm{H}_{2}\right)_{8}$ would metallize around $250 \mathrm{GPa}$. Subsequent experiments were able to refine the crystal structures of the hydrides of xenon at various pressures. ${ }^{[26}$ Between 4.8-7.1 GPa the structure was indexed as having the $P \overline{3} m 1$ spacegroup, however the site occupancy, and potentially stoichiometry, changed as a function of pressure. Its structural evolution at higher pressures was consistent with the previously reported $R 3$ phase. Decompression at low temperature to ambient pressures illustrated that $\mathrm{Xe}-\mathrm{H}_{2}$ phases can be stable up to $90 \mathrm{~K}$.

CSP techniques have also been used to predict the most stable $\mathrm{XeH}_{n}(n=1-8)$ structures up to $300 \mathrm{GPa} \cdot{ }^{433}$ Only $\mathrm{XeH}_{2}$ and $\mathrm{XeH}_{4}$ were found to be thermodynamically stable, and all other stoichiometries were metastable. $\mathrm{Cmca}-\mathrm{XeH}_{2}$ was stable with respect to the elemental phases already at $1 \mathrm{GPa}$, and it was the lowest point on the convex hull up to $300 \mathrm{GPa}$. A Bader analysis revealed pressure induced charge transfer from $\mathrm{Xe}$ to $\mathrm{H}$ atoms. $\mathrm{XeH}_{4}$ assumed $\mathrm{Amm} 2$ symmetry below, and $C m$ symmetry above $100 \mathrm{GPa}$. With the exception of $\mathrm{XeH}$, all of the phases contained $\mathrm{H}_{2}$ molecules. Whereas hybrid functionals showed that $\mathrm{XeH}_{2}$ metalized near $300 \mathrm{GPa}, \mathrm{XeH}$ was found to be metallic already at atmospheric pressures. The $T_{c} \mathrm{~s}$ of $\mathrm{XeH}$ at $100 \mathrm{~K}$ and $\mathrm{XeH}_{2}$ at $400 \mathrm{GPa}$ were both estimated to be near $30 \mathrm{~K}$, and they decreased with increasing pressure. 


\section{Radon}

To the best of our knowledge, the hydrides of radon have not yet been studied theoretically nor experimentally.

\section{Conclusions}

In just over a decade the phase diagrams of most binary hydrides as a function of stoichiometry and pressure have been investigated via first-principles calculations. Many of these studies have been carried out using state-of-the-art crystal structure prediction techniques. Because experiments at very high pressures can be difficult to carry out, at first only a handful of high hydride phases were synthesized. However, intense efforts have resulted in the synthesis of a number of hydrides with unique structures and stoichiometries under pressure including those of lithium, 66

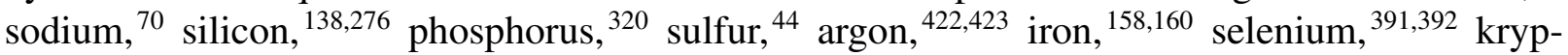

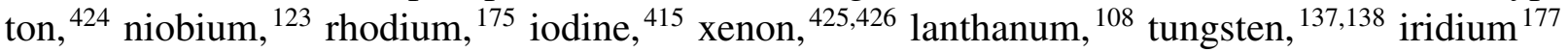
and platinum. ${ }^{198}$ Superconductivity has been measured in some of these (phosphorus, sulfur, platinum), and predicted in others, as shown in Table 1 . In particular, the discovery of conventional phonon-mediated superconductivity in compressed hydrogen sulfide at $203 \mathrm{~K}$ and $150 \mathrm{GPa}, \underline{44}$ which occurred via a synergistic feedback loop between theory and experiment, ${ }^{[50}$ was a spectacular breakthrough in the field. Theoretical investigations have attempted to understand the factors responsible for high temperature superconductivity in the compressed high hydrides, $\stackrel{104434}{1}$ and materials that are superconducting at room temperature (but very high pressures) ${ }^{104} 107$ have even been predicted. Superhydride research is blossoming, and it is therefore likely that it will lead to the discovery of quite interesting materials. 
Table 1: A compilation of the highest computationally estimated $T_{c}(\mathrm{~K})$ values for hydrides of the main group and transition metal elements that are available, along with the stoichiometry, space-group and pressure at which these values were obtained.

\begin{tabular}{|c|c|c|c|c|c|}
\hline Group & System & Pressure $(\mathrm{GPa})$ & $\mathrm{T}_{c}(\mathrm{~K})$ & $\mu^{*}$ & Space Group \\
\hline \multirow[t]{2}{*}{1} & $\mathrm{LiH}_{6}$ & 300 & $82^{d, 65}$ & 0.13 & $R \overline{3} m$ \\
\hline & $\mathrm{KH}_{6}$ & 230 & $59-70^{d, 71}$ & $0.13-0.10$ & $C 2 / c$ \\
\hline \multirow[t]{5}{*}{2} & $\mathrm{BeH}_{2}$ & 365 & $97^{d, 86}$ & 0.10 & $\mathrm{P} 4 / \mathrm{nmm}$ \\
\hline & $\mathrm{MgH}_{6}$ & 400 & $271^{e, 88}$ & 0.12 & Im $\overline{3} m$ \\
\hline & $\mathrm{CaH}_{6}$ & 150 & $220-235^{b, 89}$ & $0.13-0.10$ & $\operatorname{Im} \overline{3} m$ \\
\hline & $\mathrm{SrH}_{6}$ & 250 & $156^{b}$ & 0.10 & $R \overline{3} m$ \\
\hline & $\mathrm{BaH}_{6}$ & 100 & $30-38^{d, 92}$ & $0.13-0.10$ & $\mathrm{P} 4 / \mathrm{mmm}$ \\
\hline \multirow[t]{4}{*}{3} & $\mathrm{ScH}_{9}$ & 300 & $233^{b, 105}$ & 0.10 & $I 4_{1} m d$ \\
\hline & $\mathrm{YH}_{10}$ & 250 & $305-326^{b, 107}$ & $0.13-0.10$ & $F m \overline{3} m$ \\
\hline & $\mathrm{LaH}_{10}$ & 200 & $288^{b, 104}$ & 0.10 & $F m \overline{3} m$ \\
\hline & $\mathrm{CeH}_{10}$ & 200 & $50-55^{b, e, 104}$ & $0.13-0.10$ & $F m \overline{3} m$ \\
\hline \multirow[t]{3}{*}{4} & $\mathrm{TiH}_{2}$ & $1 \mathrm{~atm}$ & $7^{c, 110}$ & 0.10 & $F m \overline{3} m$ \\
\hline & $\mathrm{ZrH}$ & 120 & $11^{d, 116}$ & 0.10 & $\mathrm{Cmcm}$ \\
\hline & $\mathrm{HfH}_{2}$ & 260 & $11-13^{d, 117}$ & $0.13-0.10$ & $P 2_{1} / m$ \\
\hline \multirow[t]{3}{*}{5} & $\mathrm{VH}_{2}$ & 60 & $4^{d, 119}$ & 0.10 & Pnma \\
\hline & $\mathrm{NbH}_{4}$ & 300 & $50^{b, 122}$ & 0.10 & $I 4 / \mathrm{mmm}$ \\
\hline & $\mathrm{TaH}_{6}$ & 300 & $124-136^{d, 124}$ & $0.13-0.10$ & $F d d 2$ \\
\hline 6 & $\mathrm{CrH}_{3}$ & 81 & $37^{d, 133}$ & 0.10 & $P 6_{3} / m m c$ \\
\hline 7 & $\mathrm{TcH}_{2}$ & 200 & $7-11^{d, 145}$ & $0.13-0.10$ & $I 4 / \mathrm{mmm}$ \\
\hline \multirow[t]{3}{*}{8} & $\mathrm{FeH}_{5}$ & 130 & $51^{d, 161}$ & 0.10 & $I 4 / \mathrm{mmm}$ \\
\hline & $\mathrm{RuH}_{3}$ & 100 & $4^{d, 163}$ & 0.10 & $\operatorname{Pr} \overline{3} m$ \\
\hline & $\mathrm{OsH}$ & 100 & $2^{d, 165}$ & 0.10 & $F m \overline{3} m$ \\
\hline \multirow[t]{2}{*}{9} & $\mathrm{RhH}$ & 4 & $\sim 2.5^{d, e, 195}$ & 0.13 & $F m \overline{3} m$ \\
\hline & $\mathrm{IrH}$ & 80 & $7^{d, 195}$ & 0.13 & $F m \overline{3} m$ \\
\hline \multirow[t]{2}{*}{10} & $\mathrm{PdH} / \mathrm{PdD} / \mathrm{PdT}$ & $1 \mathrm{~atm}$ & $47 / 34 / 30^{b, 184}$ & 0.085 & $F m \overline{3} m$ \\
\hline & $\mathrm{PtH}$ & 77 & $25^{d, 195}$ & 0.13 & $F m \overline{3} m$ \\
\hline 11 & $\mathrm{AuH}$ & 220 & $21^{d, 195}$ & 0.13 & $F m \overline{3} m$ \\
\hline \multirow[t]{4}{*}{13} & $\mathrm{~B}_{2} \mathrm{H}_{6}$ & 360 & $90-125^{d, 218}$ & $0.20-0.13$ & Pbcn \\
\hline & $\mathrm{AlH}_{3}\left(\mathrm{H}_{2}\right)$ & 250 & $132-146^{d, 240}$ & $0.13-0.10$ & $P 2_{1} / m$ \\
\hline & $\mathrm{GaH}_{3}$ & 120 & $90-123^{b, 245}$ & $0.20-0.10$ & $P m \overline{3} n$ \\
\hline & $\mathrm{InH}_{3}$ & 200 & $34-41^{d, 251}$ & $0.13-0.10$ & $R \overline{3}$ \\
\hline \multirow[t]{4}{*}{14} & $\mathrm{Si}_{2} \mathrm{H}_{6}$ & 275 & $139-153^{d, 284}$ & $0.13-0.10$ & $\operatorname{Pm} \overline{3} m$ \\
\hline & $\mathrm{GeH}_{3}$ & 180 & $140^{d, 302}$ & 0.13 & $P m \overline{3} n$ \\
\hline & $\mathrm{SnH}_{14}$ & 300 & $86-97^{d, 307}$ & $0.13-0.10$ & $C 2 / m$ \\
\hline & $\mathrm{PbH}_{4}\left(\mathrm{H}_{2}\right)_{2}$ & 230 & $107^{d, 296}$ & 0.10 & $C 2 / m$ \\
\hline \multirow[t]{2}{*}{15} & $\mathrm{PH}_{2}$ & 270 & $87^{a, e, 321}$ & - & $I 4 / \mathrm{mmm}$ \\
\hline & $\mathrm{AsH}_{8}$ & 450 & $151^{d, 326}$ & 0.10 & $C 2 / c$ \\
\hline
\end{tabular}




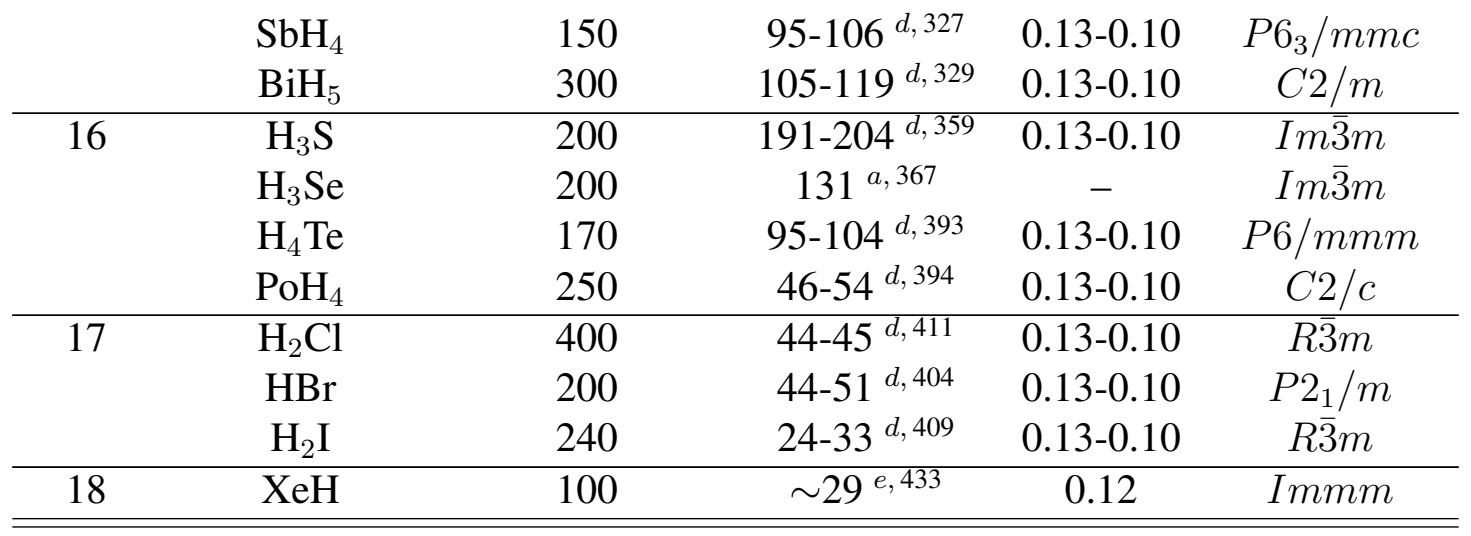

${ }^{a} T_{c}$ was predicted using SCDFT.

${ }^{b} T_{c}$ was calculated by solving the Eliashberg equations numerically.

${ }^{c} T_{c}$ was calculated using the simplified Allen-Dynes formula.

${ }^{d} T_{c}$ was calculated using the Allen-Dynes modified McMillan equation.

${ }^{e}$ These values were estimated from plots found in the original papers. 


\section{Acknowledgements}

We acknowledge the NSF (DMR-1505817) for financial, and the Center for Computational Research (CCR) at SUNY Buffalo for computational support. T.B. acknowledges financial support from the Department of Energy National Nuclear Security Administration under Award Number de-na0002006. 


\section{References}

1. Wigner, E.; Huntington, H. B. On the Possibility of a Metallic Modification of Hydrogen. J. Chem. Phys. 1935, 3, 764-770.

2. Ashcroft, N. W. Metallic Hydrogen: A High-Temperature Superconductor? Phys. Rev. Lett. 1968, 21, 1748-1749.

3. Ashcroft, N. W. Hydrogen Dominant Metallic Alloys: High Temperature Superconductors? Phys. Rev. Lett. 2004, 92, 187002.

4. Ashcroft, N. W. Bridgman's High-pressure Atomic Destructibility and Its Growing Legacy of Ordered States. J. Phys.: Condens. Matter 2004, 16, S945-S952.

5. Carlsson, A. E.; Ashcroft, N. W. Approaches for Reducing the Insulator-Metal Transition Pressure in Hydrogen. Phys. Rev. Lett. 1983, 50, 1305-1308.

6. Hemley, R. J. Effects of High Pressure on Molecules. Annu. Rev. Phys. Chem. 2000, 51, 763-800.

7. Song, Y. New Perspectives on Potential Hydrogen Storage Materials Using High Pressure. Phys. Chem. Chem. Phys. 2013, 15, 14524-14547.

8. Grochala, W.; Hoffmann, R.; Feng, J.; Ashcroft, N. W. The Chemical Imagination at Work in Very Tight Places. Angew. Chem. Int. Ed. 2007, 46, 3620-3642.

9. Goncharov, A. F.; Howie, R. T.; Gregoryanz, E. Hydrogen at Extreme Pressures. Low Temp. Phys. 2013, 39, 402-408.

10. Bhardwaj, P.; Singh, S. Pressure Induced Structural Transitions-A review. Cent. Eur. J. Chem. 2012, 10, 1391-1422.

11. Dubrovinsky, L.; Dubrovinskaia, N. Comprehensive Inorganic Chemistry II (Second Edition): From Elements to Applications; Elsevier, 2013; Vol. 2; pp 223-239.

12. McMillan, P. F. Comprehensive Inorganic Chemistry II (Second Edition): From Elements to Applications; Elsevier, 2013; Vol. 2; pp 17-46.

13. Klug, D. D.; Yao, Y. Metallization of Solid Hydrogen: The Challenges and Possible Solutions. Phys. Chem. Chem. Phys. 2011, 13, 16999-17006.

14. Naumov, I. I.; Hemley, R. J. Aromaticity, Closed-Shell Effects, and Metallization of Hydrogen. Acc. Chem. Res. 2014, 47, 3551-3559.

15. Zurek, E.; Grochala, W. Predicting Crystal Structures and Properties of Matter under Extreme Conditions via Quantum Mechanics: the Pressure is On. Phys. Chem. Chem. Phys. 2015, 17, 2917-2934.

16. Zurek, E. In Handbook of Solid State Chemistry; Dronskowski, R., Ed.; Wiley-VCH Verlag $\mathrm{GmbH} \& \mathrm{Co}, 2017$; Vol. 5; pp 571-605. 
17. Hermann, A. In Reviews in Computational Chemistry; Parrill, A. L., Lipkowitz, K. B., Eds.; John Wiley \& Sons, Inc.: Hoboken, New Jersey, 2017; pp 1-41.

18. Zurek, E. In Reviews in Computational Chemistry; Parrill, A. L., Lipkowitz, K. B., Eds.; John Wiley \& Sons, Inc.: Hoboken, New Jersey, 2016; Vol. 29; pp 274-326.

19. Woodley, S. M.; Catlow, R. Crystal Structure Prediction from First Principles. Nat. Mater. 2008, 7, 937-946.

20. Schön, J. C.; Doll, K.; Jansen, M. Predicting Solid Compounds via Global Exploration of the Energy Landscape of Solids on the Ab Initio Level without Recourse to Experimental Information. Phys. Status Solidi B 2010, 247, 23-29.

21. Revard, B. C.; Tipton, W. W.; Hennig, R. G. Structure and Stability Prediction of Compounds with Evolutionary Algorithms; Topics in Current Chemistry; Springer Berlin Heidelberg, 2014; pp 181-222.

22. Oganov, A. R., Ed. Modern Methods of Crystal Structure Prediction; Wiley-VCH, 2011.

23. Jansen, M. Conceptual Inorganic Materials Discovery - A Road Map. Adv. Mater. 2015, 27, 3229-3242.

24. Oganov, A. R.; Lyakhov, A. O.; Valle, M. How Evolutionary Crystal Structure Prediction Works - and Why. Acc. Chem. Res. 2011, 44, 227-237.

25. Zhang, L.; Wang, Y.; Lv, J.; Ma, Y. Materials Discovery at High Pressures. Nature Rev. Mater. 2017, 2, 17005.

26. Wang, Y.; Ma, Y. Perspective: Crystal Structure Prediction at High Pressures. J. Chem. Phys. 2014, 140, 040901.

27. Pickard, C. J.; Needs, R. J. Structures at High Pressure from Random Searching. Phys. Status Solidi B 2009, 246, 536-540.

28. Needs, R. J.; Pickard, C. J. Perspective: Role of Structure Prediction in Materials Discovery and Design. APL Mater. 2016, 4, 053210.

29. Pickard, C. J.; Needs, R. J. Ab Initio Random Structure Searching. J. Phys.: Condens. Matter 2011, 23, 053201.

30. Lonie, D. C.; Zurek, E. XtalOpt: An Open-Source Evolutionary Algorithm for Crystal Structure Prediction. Comput. Phys. Commun. 2011, 182, 372-387.

31. Tipton, W. W.; Bealing, C. R.; Mathew, K.; Hennig, R. G. Structures, Phase Stabilities, and Electrical Potentials of Li-Si Battery Anode Materials. Phys. Rev. B 2013, 87, 184114.

32. Glass, C. W.; Oganov, A. R.; Hansen, N. USPEX-Evolutionary Crystal Structure Prediction. Comput. Phys. Commun. 2006, 175, 713-720. 
33. Kolmogorov, A. N.; Shah, S.; Margine, E. R.; et al. New Superconducting and Semiconducting Fe-B Compounds Predicted with an Ab Initio Evolutionary Search. Phys. Rev. Lett. 2010, 105, 217003.

34. Bahmann, S.; Kortus, J. EVO-Evolutionary Algorithm for Crystal Structore Prediction. Comput. Phys. Commun. 2013, 184, 1618-1625.

35. Trimarchi, G.; Zunger, A. Global Space-Group Optimization Problem: Finding the Stablest Crystal Structure Without Constraints. Phys. Rev. B. 2007, 75, 104113.

36. Abraham, N. L.; Probert, M. I. J. A Periodic Genetic Algorithm with Real-Space Representation for Crystal Structure and Polymorph Prediction. Phys. Rev. B. 2006, 73, 224104.

37. Fadda, A.; Fadda, G. An Evolutionary Algorithm for the Prediction of Crystal Structures. Phys. Rev. B 2010, 82, 104105.

38. Wu, S. Q.; Ji, M.; Wang, C. Z.; et al. An Adaptive Genetic Algorithm for Crystal Structure Prediction. J. Phys.: Condens. Matter 2014, 26, 035402.

39. Wang, Y.; Lv, J.; Zhu, L.; Ma, Y. Crystal Structure Prediction via Particle-Swarm Optimization. Phys. Rev. B 2010, 82, 094116.

40. Wales, D. J.; Doye, J. P. K. Global Optimization by Basin-Hopping and the Lowest Energy Structures of Lennard-Jones Clusters Containing up to 110 atoms. J. Phys. Chem. A 1997, $101,5111-5116$.

41. Goedecker, S. Minima Hopping: An Efficient Search Method for the Global Minimum of the Potential Energy Surface of Complex Molecular Systems. J. Chem. Phys. 2004, 120, 9911-9917.

42. Laio, A.; Parrinello, M. Escaping Free-Energy Minima. Proc. Natl. Acad. Sci. 2002, 99, $12562-12566$.

43. Kirkpatrick, S.; Gelatt, C. D.; Vecchi, M. P. Optimization by Simulated Annealing. Science 1983, 220, 671-680.

44. Drozdov, A. P.; Eremets, M. I.; Troyan, I. A.; Ksenofontov, V.; Shylin, S. I. Conventional Superconductivity at 203 Kelvin at High Pressures in the Sulfur Hydride System. Nature 2015, 525, 73-76.

45. Zurek, E. Hydrides of the Alkali Metals and Alkaline Earth Metals under Pressure. Comments Inorg. Chem. 2016, 37, 78-98.

46. Shamp, A.; Zurek, E. Superconductivity in Hydrides Doped with Main Group Elements under Pressure. Nov. Supercond. Mater. 2017, 3, 14-22.

47. Duan, D.; Liu, Y.; Ma, Y.; et al. Structure and Superconductivity of Hydrides at High Pressures. Natl. Sci. Rev. 2016, 4, 121-135. 
48. Wang, H.; Li, X.; Gao, G.; Li, Y.; Ma, Y. Hydrogen-Rich Superconductors at High Pressures. Wiley Interdiscip. Rev. Comput. Mol. Sci. 2017, 8, 1-13.

49. Struzhkin, V. V. Superconductivity in Compressed Hydrogen-Rich Materials: Pressing on Hydrogen. Physica C 2015, 514, 77-85.

50. Yao, Y.; Tse, J. S. Superconducting Hydrogen Sulfide. Chem. Eur. J. 2017, 24, 1769-1778.

51. Bose, S. K.; Kortus, J. In Vibronic and Electron-Phonon Interactions and Their Role in Modern Chemistry and Physics; Sabin, J. R., Ed.; 2009; pp 1-62.

52. van Setten, M. J.; Popa, V. A.; de Wijs, G. A.; Brocks, G. Electronic Structure and Optical Properties of Lightweight Metal Hydrides. Phys. Rev. B 2007, 75, 035204.

53. Lebègue, S.; Arnaud, B.; Alouani, M.; Bloechl, P. E. Implementation of an All-Electron GW Approximation Based On the Projector Augmented Wave Method without Plasmon Pole Approximation: Application to $\mathrm{Si}, \mathrm{SiC}, \mathrm{AlAs}$, InAs, $\mathrm{NaH}$ and KH. Phys. Rev. B 2003, 67, 155208.

54. Duclos, S. J.; Vohra, Y. K.; Ruoff, A. L.; Filipek, S.; Baranowski, B. High-Pressure Studies of NaH to 54 GPa. Phys. Rev. B 1987, 36, 7664-7667.

55. Hochheimer, H. D.; Strössner, K.; Hönle, W.; Baranowski, B.; Filipek, F. High-Pressure $\mathrm{X}$-ray Investigation of the Alkali Hydrides $\mathrm{NaH}, \mathrm{KH}, \mathrm{RbH}$, and CsH. Z. Phys. Chem. 1985, $143,139-144$.

56. Ghandehari, K.; Luo, H.; Ruoff, A. L.; Trail, S. S.; DiSalvo, F. J. New High-Pressure Crystal Structure and Equation of State of Cesium Hydride to $253 \mathrm{GPa}$. Phys. Rev. Lett. 1995, 74, 2264-2267.

57. Ghandehari, K.; Luo, H.; Ruoff, A. L.; Trail, S. S.; DiSalvo, F. J. Crystal Structure and Band Gap of Rubidium Hydride to 120 GPa. Mod. Phys. Lett. B. 1995, 9, 1133-1140.

58. Ahuja, R.; Eriksson, O.; Wills, J. M.; Johansson, B. Theoretical High-pressure Studies of Caesium Hydride. J. Phys.: Condens. Matter 1998, 10, L153-L158.

59. Ahuja, R.; Eriksson, O.; Johansson, B. Theoretical Search for the CrB-Type High-pressure Phase in LiH, NaH, KH and RbH. Physica B 1999, 265, 87-91.

60. Hooper, J.; Baettig, P.; Zurek, E. Pressure Induced Structural Transitions in KH, RbH and CsH. J. Appl. Phys. 2012, 111, 112611.

61. Lebègue, S.; Alouani, M.; Arnaud, B.; Pickett, W. E. Pressure-Induced Simultaneous Metal-Insulator and Structural-Phase Transitions in LiH: A Quasiparticle Study. EPL 2003, $63,562-568$.

62. Ghandehari, K.; Luo, H.; Ruoff, A. L.; Trail, S. S.; DiSalvo, F. J. Band Gap and Index of Refraction of CsH to 251 GPa. Solid State Commun. 1995, 95, 385-388. 
63. Hooper, J.; Zurek, E. Lithium Subhydrides Under Pressure and their Superatom-Like Building Blocks. ChemPlusChem 2012, 77, 969-972.

64. Zurek, E.; Hoffmann, R.; Ashcroft, N. W.; Oganov, A. R.; Lyakhov, A. O. A Little Bit of Lithium Does a Lot for Hydrogen. Proc. Natl. Acad. Sci. U.S.A. 2009, 106, 17640-17643.

65. Xie, Y.; Li, Q.; Oganov, A. R.; Wang, H. Superconductivity of Lithium-Doped Hydrogen Under High Pressure. Acta Cryst. 2014, C70, 104-111.

66. Pépin, C.; Loubeyre, P.; Occelli, F.; Dumas, P. Synthesis of Lithium Polyhydrides above $130 \mathrm{GPa}$ at 300 K. Proc. Natl. Acad. Sci. U.S.A. 2015, 112, 7673-7676.

67. Dion, M.; Rydberg, H.; Schröder, E.; Langreth, D. C.; Lundqvist, B. I. Van der Waals Density Functional for General Geometries. Phys. Rev. Lett. 2004, 92, 246401.

68. Chen, Y.; Geng, H. Y.; Yan, X.; et al. Prediction of Stable Ground-State Lithium Polyhydrides under High Pressures. Inorg. Chem. 2017, 56, 3867-3874.

69. Baettig, P.; Zurek, E. Pressure-Stabilized Sodium Polyhydrides, $\mathrm{NaH}_{n}(n>1)$. Phys. Rev. Lett. 2011, 106, 237002.

70. Struzhkin, V. V.; Kim, D. Y.; Stavrou, E.; et al. Synthesis of Sodium Polyhydrides at High Pressures. Nat. Commun. 2016, 7, 12267.

71. Zhou, D.; Jin, X.; Meng, X.; et al. Ab Initio Study Revealing a Layered Structure in Hydrogen-Rich $\mathrm{KH}_{6}$ under High Pressure. Phys. Rev. B 2012, 86, 014118.

72. Hooper, J.; Zurek, E. High Pressure Potassium Polyhydrides: A Chemical Perspective. J. Phys. Chem. C 2012, 116, 13322-13328.

73. Hooper, J.; Zurek, E. Rubidium Polyhydrides Under Pressure: Emergence of the Linear $\mathrm{H}_{3}^{-}$ Species. Chem. Eur. J. 2012, 18, 5013-5021.

74. Shamp, A.; Hooper, J.; Zurek, E. Compressed Cesium Polyhydrides: $\mathrm{Cs}^{+}$Sublattices and $\mathrm{H}_{3}^{-}$Three-Connected Nets. Inorg. Chem. 2012, 51, 9333-9342.

75. Wang, Z.; Yao, Y.; Zhu, L.; et al. Metallization and Superconductivity of $\mathrm{BeH}_{2}$ under High Pressure. J. Chem. Phys. 2014, 140, 124707.

76. Zhang, L.; Wang, Y.; Cui, T.; et al. $\mathrm{CaCl}_{2}$-Type High-Pressure Phase of Magnesium Hydride Predicted by Ab Initio Phonon calculations. Phys. Rev. B 2007, 75, 144109.

77. Vajeeston, P.; Ravindran, P.; Kjekshus, A.; Fjellvåg, H. Structural Stability of $\mathrm{BeH}_{2}$ at High Pressures. Appl. Phys. Lett. 2004, 84, 34-36.

78. Vajeeston, P.; Ravindran, P.; Hauback, B. C.; et al. Structural Stability and Pressure-Induced Phase Transitions in $\mathrm{MgH}_{2}$. Phys. Rev. B 2006, 73, 224102.

79. Vajeeston, P.; Ravindran, P.; Kjekshus, A.; Fjellvåg, H. Pressure-Induced Structural Transitions in $\mathrm{MgH}_{2}$. Phys. Rev. Lett. 2002, 89, 175506. 
80. Tse, J. S.; Klug, D. D.; Desgreniers, S.; et al. Structural Phase Transition in $\mathrm{CaH}_{2}$ at High Pressures. Phys. Rev. B 2007, 75, 134108.

81. Li, B.; Li, Y.; Yang, K.; et al. Raman Evidence of a New High-Pressure Phase in Calcium Hydride. J. Phys.: Condens. Matter 2007, 19, 226205.

82. Smith, J. S.; Desgreniers, S.; Klug, D. D.; Tse, J. S. High-density Strontium Hydride: An Experimental and Theoretical Study. Solid State Commun. 2009, 149, 830-834.

83. Tse, J. S.; Song, Z.; Yao, Y.; et al. Structure and Electronic Properties of $\mathrm{BaH}_{2}$ at High Pressure. Solid State Commun. 2009, 149, 1944-1946.

84. Chen, C.; Tian, F.; Wang, L.; et al. New High-Pressure Phase of $\mathrm{BaH}_{2}$ Predicted by Ab Initio Studies. J. Phys.: Condens. Matter 2010, 22, 225401.

85. Zhang, C.; Chen, X. J.; Zhang, R. Q.; Lin, H. Q. Chemical Trend of Pressure-Induced Metallization in Alkaline Earth Hydrides. J. Phys. Chem. C 2010, 114, 14614-14617.

86. Yu, S.; Zeng, Q.; Oganov, A. R.; et al. Exploration of Stable Compounds, Crystal Structures, and Superconductivity in the Be-H system. AIP Adv. 2014, 4, 107118.

87. Lonie, D. C.; Hooper, J.; Altintas, B.; Zurek, E. Metallization of Magnesium Polyhydrides under Pressure. Phys. Rev. B 2013, 87, 054107.

88. Feng, X.; Zhang, J.; Gao, G.; Liu, H.; Wang, H. Compressed Sodalite-Like $\mathrm{MgH}_{6}$ as a Potential High-Temperature Superconductor. RSC Adv. 2015, 5, 59292-59296.

89. Wang, H.; Tse, J. S.; Tanaka, K.; Iitaka, T.; Ma, Y. Superconductive Sodalite-Like Clathrate Calcium Hydride at High pressures. Proc. Natl. Acad. Sci. U.S.A. 2012, 109, 6463-6466.

90. Hooper, J.; Terpstra, T.; Shamp, A.; Zurek, E. The Composition and Constitution of Compressed Strontium Polyhydrides. J. Phys. Chem. C 2014, 118, 6433-6447.

91. Wang, Y.; Wang, H.; Tse, J. S.; Iitaka, T.; Ma, Y. Structural Morphologies of High-Pressure Polymorphs of Strontium Hydrides. Phys. Chem. Chem. Phys. 2015, 17, 19379-19385.

92. Hooper, J.; Altintas, B.; Shamp, A.; Zurek, E. Polyhydrides of the Alkaline Earth Metals: A Look at the Extremes under Pressure. J. Phys. Chem. C 2013, 117, 2982-2992.

93. Pakornchote, T.; Pinsook, U.; Bovornratanaraks, T. The hcp to fcc Transformation Path of Scandium Trihydride under High Pressure. J. Phys.: Condens. Matter 2014, 26, 025405.

94. de Almeida, J. S.; Kim, D. Y.; Ortiz, C.; Klintenberg, M.; Ahuja, R. On the Dynamical Stability and Metallic Behavior of $\mathrm{YH}_{3}$ under Pressure. Appl. Phys. Lett. 2009, 94, 251913.

95. Kim, D. Y.; Scheicher, R. H.; Mao, H. k.; Kang, T. W.; Ahuja, R. General Trend for Pressurized Superconducting Hydrogen-Dense Materials. Proc. Natl. Acad. Sci. U.S.A. 2010, 107, 2793-2796. 
96. Wei, Y. K.; Yuan, J. N.; Khan, F. I.; et al. Pressure Induced Superconductivity and Electronic Structure Properties of Scandium Hydrides Using First-Principles Calculations. RSC Adv. 2016, 6, 81534-81541.

97. Kim, D. Y.; Scheicher, R. H.; Ahuja, R. Predicted High-Temperature Superconducting State in the Hydrogen-Dense Transition-Metal Hydride $\mathrm{YH}_{3}$ at $40 \mathrm{~K}$ and $17.7 \mathrm{GPa}$. Phys. Rev. Lett. 2009, 103, 077002.

98. Durajski, A. P.; Szczęśniak, R. Properties of the Pressure-Induced Superconducting State in Trihydrides $\mathrm{ScH}_{3}$ and $\mathrm{LaH}_{3}$. Supercond. Sci. Technol. 2014, 27, 115012.

99. Ye, X.; Hoffmann, R.; Ashcroft, N. W. Theoretical Study of Phase Separation of Scandium Hydrides under High Pressure. J. Phys. Chem. C 2015, 119, 5614-5625.

100. Yao, Y.; Klug, D. D. Consecutive Peierls distortions and high-pressure phase transitions in $Y H_{3}$. Phys. Rev. B 2010, 81, 140104(R).

101. Liu, L. L.; Sun, H. J.; Wang, C. Z.; Lu, W. C. High-pressure structures of yttrium hydrides. J. Phys.: Condens. Matter 2017, 29, 325401.

102. Abe, K. Hydrogen-Rich Scandium Compounds at High Pressures. Phys. Rev. B 2017, 96, 144108.

103. Qian, S.; Sheng, X.; Yan, X.; Chen, Y.; Song, B. Theoretical Study of Stability and Superconductivity of $\mathrm{ScH}_{n}(\mathrm{n}=4-8)$ at High Pressure. Phys. Rev. B 2017, 96, 094513.

104. Peng, F.; Sun, Y.; Pickard, C. J.; et al. Hydrogen Clathrate Structures in Rare Earth Hydrides at High Pressures: Possible Route to Room-Temperature Superconductivity. Phys. Rev. Lett. 2017, 119, 107001.

105. Ye, X.; Zarifi, N.; Zurek, E.; Hoffmann, R.; Ashcroft, N. W. High Hydrides of Scandium under Pressure: Potential Superconductors. J. Phys. Chem. C 2018, 122, 6298-6309.

106. Li, Y.; Hao, J.; Liu, H.; et al. Pressure-Stabilized Superconductive Yttrium Hydrides. Sci. Rep. 2015, 5, 9948.

107. Liu, H.; Naumov, I. I.; Hoffmann, R.; Ashcroft, N. W.; Hemley, R. J. Potential High-T Superconducting Lanthanum and Yttrium Hydrides at High Pressure. Proc. Natl. Acad. Sci. U.S.A. 2017, 114, 6990-6995.

108. Geballe, Z. M.; Liu, H.; Mishra, A. K.; et al. Synthesis and Stability of Lanthanum Superhydrides. Angew. Chem. Int. Ed. 2018, 57, 688-692.

109. Bashkin, I. O.; Nefedova, M. V.; Tissen, V. G.; Ponyatovskii, E. G. Superconductivity in the Ti-D System under Pressure. Phys. Solid State 1998, 40, 1950-1952.

110. Shanavas, K. V.; Lindsay, L.; Parker, D. S. Electronic Structure and Electron-Phonon Coupling in $\mathrm{TiH}_{2}$. Sci. Rep. 2016, 6, 28102. 
111. Kalita, P. E.; Sinogeikin, S. V.; Lipinska-Kalita, K.; et al. Equation of State of $\mathrm{TiH}_{2}$ up to 90 GPa: A Synchrotron X-Ray Diffraction Study and Ab Initio Calculations. J. Appl. Phys. 2010, 108, 043511.

112. Gao, G.; Bergara, A.; Liu, G.; Ma, Y. Pressure Induced Phase Transitions in $\mathrm{TiH}_{2}$. J. Appl. Phys. 2013, 113, 103512.

113. Maimaitiyili, T; Steuwer, A.; Blomqvist, J.; et al. Observation of the $\delta$ to $\varepsilon \mathrm{Zr}-\mathrm{Hydride}$ Transition by In Situ Synchrotron X-Ray Diffraction. Cryst. Res. Technol. 2016, 51, 663670.

114. Huang, X.; Duan, D.; Li, F.; et al. Structural Stability and Compressive Behavior of $\mathrm{ZrH}_{2}$ under Hydrostatic Pressure and Nonhydrostatic Pressure. RSC Adv. 2014, 4, 46780-46786.

115. Zhu, W.; Wang, R.; Shu, G.; Wu, P.; Xiao, H. First-Principles Study of Different Polymorphs of Crystalline Zirconium Hydride. J. Phys. Chem. C 2010, 114, 22361-22368.

116. Li, X. F.; Hu, Z. Y.; Huang, B. Phase Diagram and Superconductivity of Compressed Zirconium Hydrides. Phys. Chem. Chem. Phys. 2017, 19, 3538-3543.

117. Liu, Y.; Huang, X.; Duan, D.; et al. First-Principles Study on the Structural and Electronic Properties of Metallic $\mathrm{HfH}_{2}$ under Pressure. Sci. Rep. 2015, 5, 11381.

118. Wang, X.; Andrews, L. Tetrahydrometalate Species $\mathrm{VH}_{2}\left(\mathrm{H}_{2}\right), \mathrm{NbH}_{4}$, and $\mathrm{TaH}_{4}$ : Matrix Infrared Spectra and Quantum Chemical Calculations. J. Phys. Chem. A 2011, 115, 1417514183.

119. Chen, C.; Tian, F; Duan, D.; et al. Pressure Induced Phase Transition in $\mathrm{MH}_{2}(\mathrm{M}=\mathrm{V}, \mathrm{Nb})$. J. Chem. Phys. 2014, 140, 114703.

120. Ohlendorf, D.; Wicke, E. Heat Capacities between 1.5 and $16 \mathrm{~K}$ and Superconductivity of V/H and Nb/H Alloys. J. Phys. Chem. Solids 1979, 40, 721-728.

121. Gao, G.; Hoffmann, R.; Ashcroft, N. W.; et al. Theoretical Study of the Ground-State Structures and Properties of Niobium Hydrides under Pressure. Phys. Rev. B 2013, 88, 184104.

122. Durajski, A. P. Phonon-Mediated Superconductivity in Compressed $\mathrm{NbH}_{4}$ compound. Eur. Phys. J. B 2014, 87, 210.

123. Liu, G.; Besedin, S.; Irodova, A.; et al. Nb-H System at High Pressures and Temperatures. Phys. Rev. B 2017, 95, 104110.

124. Zhuang, Q.; Jin, X.; Cui, T.; et al. Pressure-Stabilized Superconductive Ionic Tantalum Hydrides. Inorg. Chem. 2017, 56, 3901-3908.

125. Kuzovnikov, M. A.; Tkacz, M.; Meng, H.; Kapustin, D. I.; Kulakov, V. I. High-Pressure Synthesis of Tantalum Dihydride. Phys. Rev. B 2017, 96, 134120. 
126. Snavely, C. A.; Vaughan, D. A. Unit Cell Dimension of Face-Centered Cubic Chromium Hydride and Space Groups of Two Chromium Hydrides. J. Am. Chem. Soc. 1949, 71, 313314.

127. Poźniak-Fabrowska, J.; Nowak, B.; Tkacz, M. Magnetic Properties of Cubic and Hexagonal Chromium Hydrides: A Comparison of the Magnetic Susceptibility with the ${ }^{53} \mathrm{Cr}\{\mathrm{NMR}\}$ Knight Shift. J. Alloys Compd. 2001, 322, 82-88.

128. Wang, X.; Andrews, L. Chromium Hydrides and Dihydrogen Complexes in Solid Neon, Argon, and Hydrogen: Matrix Infrared Spectra and Quantum Chemical Calculations. J. Phys. Chem. A 2003, 107, 570-578.

129. Wang, X.; Andrews, L. Matrix Infrared Spectra and Density Functional Theory Calculations of Molybdenum Hydrides. J. Phys. Chem. A 2005, 109, 9021-9027.

130. Wang, X.; Andrews, L. Neon Matrix Infrared Spectrum of $\mathrm{WH}_{6}$ : A Distorted Trigonal Prism Structure. J. Am. Chem. Soc. 2002, 124, 5636-5637.

131. Wang, X.; Andrews, L.; Infante, I.; Gagliardi, L. Infrared Spectra of the $\mathrm{WH}_{4}\left(\mathrm{H}_{2}\right)_{4}$ Complex in Solid Hydrogen. J. Am. Chem. Soc. 2008, 130, 1972-1978.

132. Gagliardi, L.; Pyykkö, P. How Many Hydrogen Atoms Can be Bound to a Metal? Predicted $\mathrm{MH}_{12}$ Species. J. Am. Chem. Soc. 2004, 126, 15014-15015.

133. Yu, S.; Jia, X.; Frapper, G.; et al. Pressure-Driven Formation and Stabilization of Superconductive Chromium Hydrides. Sci. Rep. 2015, 5, 17764.

134. Kuzovnikov, M. A.; Meng, H.; Tkacz, M. Nonstoichiometric Molybdenum Hydride. J. Alloys Compd. 2017, 694, 51-54.

135. Feng, X.; Zhang, J.; Liu, H.; et al. High Pressure Polyhydrides of Molybdenum: A FirstPrinciples Study. Solid State Commun. 2016, 239, 14-19.

136. Zaleski-Ejgierd, P.; Labet, V.; Strobel, T. A.; Hoffmann, R.; Ashcroft, N. W. WH under Pressure. J. Phys.: Condens. Matter 2012, 24, 155701.

137. Scheler, T.; Peng, F.; Guillaume, C. L.; et al. Nanocrystalline Tungsten Hydrides at High Pressures. Phys. Rev. B 2013, 87, 184117.

138. Strobel, T. A.; Somayazulu, M.; Hemley, R. J. Novel Pressure-Induced Interactions in Silane-Hydrogen. Phys. Rev. Lett. 2009, 103, 065701.

139. Labet, V.; Hoffmann, R.; Ashcroft, N. W. Molecular Models for $\mathrm{WH}_{6}$ under Pressure. New J. Chem. 2011, 35, 2349-2355.

140. Wang, X.; Andrews, L. Matrix Infrared Spectra and Density Functional Theory Calculations of Manganese and Rhenium Hydrides. J. Phys. Chem. A 2003, 107, 4081-4091. 
141. Antonov, V. E.; Antonova, T. E.; Chirin, N. A.; et al. T-P Phase Diagram of the Mn-H System at Pressures to 4.4 GPa and Temperatures to 1000 oC. Scripta Mater. 1996, 34, 1331-1336.

142. Fukai, Y.; Haraguchi, T.; Shinomiya, H.; Mori, K. Constitution of the Mn-H System at High Hydrogen Pressures. Scripta Mater. 2002, 46, 679-684.

143. Antonov, V. E.; Belash, I. T.; Bukov, K. G.; et al. Superconductivity of Technetium Hydrides. Fizika Metallov i Metallovedeniya 1989, 68, 1198-1200.

144. Spitsyn, V. I.; Antonov, V. E.; Balakhovskii, O. A.; et al. Structure and Superconductive Properties of the High-Pressure Phase in the Technetium-Hydrogen System. Dokl. Phys. Chem. 1982, 260, 132-135.

145. Li, X.; Liu, H.; Peng, F. Crystal Structures and Superconductivity of Technetium Hydrides under Pressure. Phys. Chem. Chem. Phys. 2016, 18, 28791-287916.

146. Degtyareva, O.; Proctor, J. E.; Guillaume, C. L.; Gregoryanz, E.; Hanfland, M. Formation of Transition Metal Hydrides at High Pressures. Solid State Commun. 2009, 149, 1583-1586.

147. Atou, T.; Badding, J. V. In Situ Diffraction Study of the Formation of Rhenium Hydride at High Pressure. J. Solid State Chem. 1995, 118, 299-302.

148. Scheler, T.; Degtyareva, O.; Gregoryanz, E. On the Effects of High Temperature and High Pressure on the Hydrogen Solubility in Rhenium. J. Chem. Phys. 2011, 135, 214501.

149. Fukai, Y.; Fukizawa, A.; Watanabe, K.; Amano, M. Hydrogen in Iron-Its Enhanced Dissolution under Pressure and Stabilization of the $\gamma$ Phase. Jpn. J. Appl. Phys. 1982, 21, L318-L320.

150. Badding, J. V.; Hemley, R. J.; Mao, H. K. High-Pressure Chemistry of Hydrogen in Metals: In Situ Study of Iron Hydride. Science 1991, 253, 421-424.

151. Yamakata, M.; Yagi, T.; Utsumi, W.; Fukai, Y. In Situ X-Ray Observation of Iron Hydride under High Pressure and High Temperature. Proc. Japan Acad. 1992, 68, 172-176.

152. Antonov, V. E.; Baier, M.; Dorner, B.; et al. High-Pressure Hydrides of Iron and Its Alloys. J. Phys.: Condens. Matter 2002, 14, 6427-6445.

153. Narygina, O.; Dubrovinsky, L. S.; McCammon, C. A.; et al. X-Ray Diffraction and Mössbauer Spectroscopy Study of fcc Iron Hydride FeH at High Pressures and Implications for the Composition of the Earth's Core. Earth Planet. Sci. Lett. 2011, 307, 409-414.

154. Isaev, E. I.; Skorodumova, N. V.; Ahuja, R.; Vekilov, Y. K.; Johansson, B. Dynamical Stability of Fe-H in the Earth's Mantle and Core Regions. Proc. Natl. Acad. Sci. U.S.A. 2007, 104, 9168-9171.

155. Bazhanova, Z. G.; Oganov, A. R.; Gianola, O. Fe-C and Fe-H Systems at Pressures of the Earth's Inner Core. Phy. Usp. 2012, 55, 489-497. 
156. Li, F.; Wang, D.; Du, H.; et al. Structural Evolution of $\mathrm{FeH}_{4}$ under High Pressure. RSC Adv. 2017, 7, 12570-12575.

157. Kvashnin, A. G.; Kruglov, I. A.; Semenok, D. V.; Oganov, A. R. Iron Superhydrides $\mathrm{FeH}_{5}$ and $\mathrm{FeH}_{6}$ : Stability, Electronic Properties, and Superconductivity. J. Phys. Chem. C 2018, 122, 4731-4736.

158. Pépin, C. M.; Dewaele, A.; Geneste, G.; Loubeyre, P.; Mezouar, M. New Iron Hydrides under High Pressure. Phys. Rev. Lett. 2014, 113, 265504.

159. Tsumuraya, T.; Matsuura, Y.; Shishidou, T.; Oguchi, T. First-Principles Study on the Structural and Magnetic Properties of Iron Hydride. J. Phys. Soc. Jpn. 2012, 81, 064707.

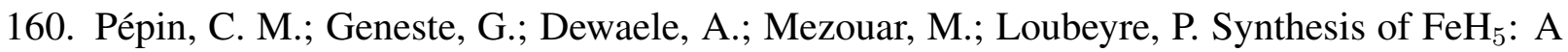
Layered Structure with Atomic Hydrogen Slabs. Science 2017, 357, 382-385.

161. Majumdar, A.; Tse, J. S.; Wu, M.; Yao, Y. Superconductivity in $\mathrm{FeH}_{5}$. Phys. Rev. B 2017, 96, 201107(R).

162. Gao, G.; Wang, H.; Zhu, L.; Ma, Y. Pressure-Induced Formation of Noble Metal Hydrides. J. Phys. Chem. C 2012, 116, 1995-2000.

163. Liu, Y.; Duan, D.; Tian, F.; et al. Stability of Properties of the Ru-H System at High Pressure. Phys. Chem. Chem. Phys. 2016, 18, 1516-1520.

164. Kuzovnikov, M. A.; Tkacz, M. Synthesis of Ruthenium Hydride. Phys. Rev. B. 2016, 93, 064103.

165. Liu, Y.; Duan, D.; Huang, X.; et al. Structures and Properties of Osmium Hydrides Under Pressure from First Principle Calculation. J. Phys. Chem. C. 2015, 119, 15905-15911.

166. Ishimatsu, N.; Shichijo, T.; Matsushima, Y.; Maruyama, H.; Matsuura, Y. et al. HydrogenInduced Modification of the Electronic Structure and Magnetic States in $\mathrm{Fe}, \mathrm{Co}$, and $\mathrm{Ni}$ Monohydrides. Phys. Rev. B 2012, 86, 104430.

167. Kuzovnikov, M. A.; Tkacz, M. High Pressure Studies of Cobalt-Hydrogen System by XRay Diffraction. J. Alloys Compd. 2015, 650, 884-886.

168. Fedotov, V. K.; Antonov, V. E.; Antonova, T. E.; Bokhenkov, E. L.; Dorner, B. et al. Atomic Ordering in the hcp Cobalt Hydrides and Deuterides. J. Alloys Compd. 1999, 291, 1-7.

169. Antonov, V. E.; Antonova, T. E.; Fedotov, V. K.; Hansen, T.; Kolesnikov, A. I. et al. Neutron Scattering Studies of $\gamma-\mathrm{CoH}$. J. Alloys Compd. 2005, 404, 73-76.

170. Białek, B.; Stępień, Z. M. Formation of Cobalt Hydrides in a High Electric Field. Vacuum 2001, 63, 241-248.

171. Uribe, E. A.; Daza, M. C.; Villaveces, J. L. $\mathrm{CoH}_{n}(\mathrm{n}=1-3)$ : Classical and Non-Classical Cobalt Polyhydride. Chem. Phys. Lett. 2010, 490, 143-147. 
172. Wang, L.; Duan, D.; Yu, H.; et al. High-Pressure Formation of Cobalt Polyhydrides: A First-Principle Study. Inorg. Chem. 2018, 57, 181-186.

173. Cui, X.; Wang, J. T.; Liang, X. X.; Zhao, G. Z. Ab Initio Studies on the Structural and Magnetic Properties of RhH. Solid State Commun. 2009, 149, 322-324.

174. Sudhapriyanga, G.; Asvinimeenaatci, A. T.; Rajeswarapalanichamy, R.; Iyakutti, K. Density Functional Study of Bulk and Surface Properties of Rhodium Hydride. Acta Phys. Pol. A 2014, 125, 29-35.

175. Li, B.; Ding, Y.; Kim, D. Y.; et al. Rhodium Dihydride $\left(\mathrm{RhH}_{2}\right)$ with High Volumetric Hydrogen Density. Proc. Natl. Acad. Sci. U.S.A. 2011, 108, 18618-18621.

176. Tkacz, M. High Pressure Studies of the Rhodium-Hydrogen System in Diamond Anvil Cell. J. Chem. Phys. 1998, 108, 2084-2087.

177. Scheler, T.; Marqués, M.; Konôpková, Z.; et al. High-Pressure Synthesis and Characterization of Iridium Trihydride. Phys. Rev. Lett. 2013, 111, 215503.

178. Zaleski-Ejgierd, P. High-Pressure Formation and Stabilization of Binary Iridium Hydrides. Phys. Chem. Chem. Phys. 2014, 16, 3220-3229.

179. Bauer, H. J.; Schmidbauer, E. "Uber den Einfluss elektrolytischer Wasserstoffbeladung auf die Magnetisierung von Nickel. Z. Phys. 1961, 164, 367-373.

180. Vargas, P.; Christensen, N. E. Band-Structure Calculations for $\mathrm{Ni}, \mathrm{Ni}_{4} \mathrm{H}, \mathrm{Ni}_{4} \mathrm{H}_{2}, \mathrm{Ni}_{4} \mathrm{H}_{3}$, and NiH. Phys. Rev. B 1987, 35, 1993-2004.

181. San, X.; Ma, Y.; Cui, T.; et al. Pressure-Induced Magnetic Transition in Metallic Nickel Hydrides by $A b$ Initio Pseudopotential Plane-Wave Calculations. Phys. Rev. B 2006, 74, 052405.

182. Houari, A.; Matar, S. F.; Eyert, V. Electronic Structure and Crystal Phase Stability of Palladium Hydrides. J. Appl. Phys. 2014, 116, 173706.

183. Stritzker, B.; Buckel, W. Superconductivity in the Palladium-Hydrogen and the PalladiumDeuterium Systems. Z. Phys. 1972, 257, 1-8.

184. Errea, I.; Calandra, M.; Mauri, F. First-Principles Theory of Anharmonicity and the Inverse Isotope Effect in Superconducting Palladium-Hydride Compounds. Phys. Rev. Lett. 2013, $111,177002$.

185. Graham, T. On the Absorption and Dialytic Separation of Gases by Colloid Spectra. Philos. Trans. R. Soc. Lond. 1866, 156, 399-439.

186. Caputo, R.; Alavi, A. Where Do the H Atoms Reside in $\mathrm{PdH}_{x}$ Systems? Mol. Phys. 2003, $101,1781-1787$. 
187. Worsham Jr., J. E.; Wilkinson, M. K.; Shull, C. G. Neutron-Diffraction Observations on the Palladium-Hydrogen and Palladium-Deuterium Systems. J. Phys. Chem. Solids 1957, 3, 303-310.

188. Schirber, J. E.; Northrup, C. J. M. Concentration Dependence of the Superconducting Transition Temperature in $\mathrm{PdH}_{x}$ and $\mathrm{PdD}_{x}$. Phys. Rev. B 1974, 10, 3818-3820.

189. Skośkiewicz, T; Szafrański, A. W.; Bujnowski, W.; Baranowski, B. Isotope Effect in the Superconducting Palladium-Hydrogen-Deuterium System. J. Phys. C: Solid State Phys. 1974, 7, 2670-2676.

190. Hemmes, H.; Driessen, A.; Griessen, R.; Gupta, M. Isotope Effects and Pressure Dependence of the $\mathrm{T}_{c}$ of Superconducting PdH and PdD Synthesized and Measured in a Diamond Anvil Cell. Phys. Rev. B 1989, 39, 4110-4118.

191. Fukai, Y.; Ōkuma, N. Formation of Superabundant Vacancies in Pd Hydride under High Hydrogen Pressures. Phys. Rev. Lett. 1994, 73, 1640-1643.

192. Feng, J.; Grochala, W.; Jaroń, T.; et al. Structures and Potential Superconductivity in $\mathrm{SiH}_{4}$ at High Pressure: En Route to Metallic Hydrogen. Phys. Rev. Lett. 2006, 96, 017006.

193. Pickard, C. J.; Needs, R. J. High-Pressure Phases of Silane. Phys. Rev. Lett. 2006, 97, 045504.

194. Eremets, M. I.; Trojan, I. A.; Medvedev, S. A.; Tse, J. S.; Yao, Y. Superconductivity in Hydrogen Dominant Materials: Silane. Science 2008, 319, 1506-1509.

195. Kim, D. Y.; Scheicher, R. H.; Pickard, C. J.; Needs, R. J.; Ahuja, R. Predicted Formation of Superconducting Platinum-Hydride Crystals Under Pressure in the Presence of Molecular Hydrogen. Phys. Rev. Lett. 2011, 107, 117002.

196. Zhou, X.-F.; Oganov, A. R.; Dong, X.; et al. Superconducting High-pressure Phase of Platinum Hydride From First Principles. Phys. Rev. B. 2011, 84, 054543.

197. Zhang, C.; Chen, X. J.; Lin, H. Q. Phase Transitions and Electron-Phonon Coupling in Platinum Hydride. J. Phys.: Condens. Matter 2012, 24, 035701.

198. Scheler, T.; Degtyareva, O.; Marqués, M.; Guillaume, C. L.; Proctor, J. E. et al. Synthesis and Properties of Platinum Hydride. Phys. Rev. B. 2011, 83, 214106.

199. Errea, I.; Calandra, M.; Mauri, F. Anharmonic Free Energies and Phonon Dispersions from the Stochastic Self-Consistent Harmonic approximation: Application to Platinum and Palladium Hydrides. Phys. Rev. B. 2014, 89, 064302.

200. Wurtz, A. Sur l'Albumine Soluble. Compt. Rend. 1844, 18, 700-702.

201. Müller, H.; Bradley, A. J. Copper Hydride and Its Crystal Structure. J. Chem. Soc. 1926, 129, 1669-1673. 
202. Goedkoop, J. A.; Andresen, A. F. The Crystal Structure of Copper Hydride. Acta Cryst. 1955, 8, 118-119.

203. Tkacz, M.; Burtovyy, R. Decomposition of the Hexagonal Copper Hydride at High Pressure. Solid State Commun. 2004, 132, 37-41.

204. Burtovyy, R.; Tkacz, M. High-Pressure Synthesis of a New Copper Hydride from Elements. Solid State Commun. 2004, 131, 169-173.

205. Donnerer, C.; Scheler, T.; Gregoryanz, E. High-Pressure Synthesis of Noble Metal Hydrides. J. Chem. Phys. 2013, 138, 134507.

206. Antonov, V. E.; Antonova, T. E.; Belash, I. T.; Gorodetskii, A. E.; Ponyatovskii, E. G. Synthesis of Gold Hydride under High Hydrogen Pressure. Dokl. Acad. Nauk. SSSR 1982, 266, 376-380.

207. Degtyareva, V. F. Crystal Structure of Gold Hydride. J. Alloys Compd. 2015, 645, S128S131.

208. Wang, X.; Andrews, L. Mercury Dihydride Forms a Covalent Molecular Solid. Phys. Chem. Chem. Phys. 2005, 7, 750-759.

209. Shayesteh, A.; Yu, S.; Bernath, P. F. Gaseous $\mathrm{HgH}_{2}, \mathrm{CdH}_{2}$, and $\mathrm{ZnH}_{2}$. Chem. Eur. J. 2005, $11,4709-4712$.

210. Greenwood, N. N. Taking Stock: The Astonishing Development of Boron Hydride Cluster Chemistry. Chem. Soc. Rev. 1992, 21, 49-57.

211. Murli, C.; Song, Y. Pressure-Induced Transformations in Diborane: A Raman Spectroscopic Study. J. Phys. Chem. B 2009, 113, 13509-13515.

212. Song, Y.; Murli, C.; Liu, Z. In Situ High-Pressure Study of Diborane by Infrared Spectroscopy. J. Chem. Phys. 2009, 131, 174506.

213. Torabi, A.; Murli, C.; Song, Y.; Staroverov, V. N. Polymorphic Transitions of Diborane at Sub- and Near-Megabar Pressures. Sci. Rep. 2015, 5, 13929.

214. Nakano, S.; Hemley, R. J.; Gregoryanz, E. A.; Goncharov, A. F.; Mao, H.-k. PressureInduced Transformations of Molecular Boron Hydride. J. Phys.: Condens. Matter 2002, 14, 10453-10456.

215. Barbee III, T. W.; McMahan, A. K.; Klepeis, J. E.; van Schilfgaarde, M. High-Pressure Boron Hydride Phases. Phys. Rev. B 1997, 56, 5148-5155.

216. Torabi, A.; Song, Y.; Staroverov, V. N. Pressure-Induced Polymorphic Transitions in Crystalline Diborane Deduced by Comparison of Simulated and Experimental Vibrational Spectra. J. Phys. Chem. C 2013, 117, 2210-2215. 
217. Yao, Y.; Hoffmann, R. $\mathrm{BH}_{3}$ under Pressure: Leaving the Molecular Diborane Motif. J. Am. Chem. Soc. 2011, 133, 21002-21009.

218. Abe, K.; Ashcroft, N. W. Crystalline Diborane at High Pressures. Phys. Rev. B. 2011, 84, 104118.

219. Hu, C.-H.; Oganov, A. R.; Zhu, Q.; et al. Pressure-Induced Stabilization and InsulatorSuperconductor Transition of BH. Phys. Rev. Lett. 2013, 110, 165504.

220. Suarez-Alcubilla, A.; Gurtubay, I. G.; Bergara, A. High Pressure Phases of Different Tetraboranes. High Press. Res. 2014, 34, 59-69.

221. Wang, X.; Andrews, L.; Tam, S.; DeRose, M. E.; Fajardo, M. E. Infrared Spectra of Aluminum Hydrides in Solid Hydrogen: $\mathrm{Al}_{2} \mathrm{H}_{4}$ and $\mathrm{Al}_{2} \mathrm{H}_{6}$. J. Am. Chem. Soc. 2003, 125, $9218-$ 9228.

222. Sinke, G. C.; Walker, L. C.; Oetting, F. L.; Stull, D. R. Thermodynamic Properties of Aluminum Hydride. J. Chem. Phys. 1967, 47, 2759-2761.

223. Vajeeston, P.; Ravindran, P.; Fjellvåg, H. Novel High Pressure Phases of $\beta-\mathrm{AlH}_{3}$ : A Density-Functional Study. Chem. Mater. 2008, 20, 5997-6002.

224. Lu, Y.-L.; Zhao, H. First-Principles Studies on the Structural Stability of $\alpha-\mathrm{AlH}_{3}$ under Pressure. Modelling Simul. Mater. Sci. Eng. 2012, 20, 085004.

225. Feng, W.; Cui, S.; Feng, M. First-Principles Study of Structural Stabilities of $\mathrm{AlH}_{3}$ under High Pressure. J. Phys. Chem. Solids 2014, 75, 803-807.

226. Graetz, J.; Chaudhuri, S.; Lee, Y.; et al. Pressure-Induced Structural and Electronic Changes in $\alpha-\mathrm{AlH}_{3}$. Phys. Rev. B 2006, 74, 214114.

227. Tkacz, M.; Palasyuk, T.; Graetz, J.; Saxena, S. High-Pressure Raman Spectroscopy Study of $\alpha$ and $\gamma$ Polymorphs of $\mathrm{AlH}_{3}$. J. Raman Spectrosc. 2008, 39, 922-927.

228. Drozd, V.; Garimella, S.; Saxena, S.; Chen, J.; Palasyuk, T. High-Pressure Raman and XRay Diffraction Study of $\beta$-and $\gamma$-Polymorphs of Aluminum Hydride. J. Phys. Chem. C 2012, 116, 3808-3816.

229. Shimura, N.; Takeichi, T.; Kume, T.; et al. High Pressure Raman and Visible Absorption Study of $\mathrm{AlH}_{3}$. J. Phys.: Conf. Ser. 2010, 215, 012047.

230. Molodets, A. M.; Shakhray, D. V.; Khrapak, A. G.; Fortov, V. E. Metallization of Aluminum Hydride $\mathrm{AlH}_{3}$ at High Multiple-Shock Pressures. Phys. Rev. B 2009, 79, 174108.

231. Besedin, S. P.; Jephcoat, A. P.; Irodova, A. V. Light-Induced Phase Transition in $\mathrm{AlD}_{3}$ at High Pressure. Phys. Rev. B 2011, 84, 104111.

232. Pickard, C. J.; Needs, R. J. Metallization of Aluminum Hydride at High Pressures: A FirstPrinciples Study. Phys. Rev. B 2007, 76, 144114. 
233. Kim, D. Y.; Scheicher, R. H.; Ahuja, R. Dynamical Stability of the Cubic Metallic Phase of $\mathrm{AlH}_{3}$ at Ambient Pressure: Density Functional Calculations. Phys. Rev. B 2008, 78, 100102(R).

234. Geshi, M.; Fukazawa, T. Pressure Induced Band Gap Opening of $\mathrm{AlH}_{3}$. Physica B 2013, $411,154-160$.

235. Wei, Y.-K.; Ge, N.-N.; Ji, G.-F.; et al. Elastic, Superconducting, and Thermodynamic Properties of the Cubic Metallic Phase of $\mathrm{AlH}_{3}$ via First-Principles Calculations. J. Appl. Phys. 2013, 114, 114905.

236. Shi, H.; Zarifi, N.; Yim, W.-L.; Tse, J. S. Electron Band Structure of the High Pressure Cubic Phase of $\mathrm{AlH}_{3}$. J. Phys. Conf. Ser. 2012, 377, 012093.

237. Goncharenko, I.; Eremets, M. I.; Hanfland, M.; Tse, J. S.; Amboage, M. et al. PressureInduced Hydrogen-Dominant Metallic State in Aluminum Hydride. Phys. Rev. Lett. 2008, $100,045504$.

238. Rousseau, B.; Bergara, A. Giant Anharmonicity Suppresses Superconductivity in $\mathrm{AlH}_{3}$ under Pressure. Phy. Rev. B 2010, 82, 104504.

239. Rousseau, B.; Bergara, A. Anharmonicity in Aluminum Hydride at High Pressures. High Press. Res. 2011, 31, 30-34.

240. Hou, P.; Zhao, X.; Tian, F.; et al. High Pressure Structures and Superconductivity of $\mathrm{AlH}_{3}$ $\left(\mathrm{H}_{2}\right)$ Predicted by First Principles. RSC Adv. 2015, 5, 5096-5101.

241. Himmel, H.-J.; Manceron, L.; Downs, A. J.; Pullumbi, P. Formation and Characterization of the Gallium and Indium Subhydride Molecules $\mathrm{Ga}_{2} \mathrm{H}_{2}$ and $\mathrm{In}_{2} \mathrm{H}_{2}$ : A Matrix Isolation Study. J. Am. Chem. Soc. 2002, 124, 4448-4457.

242. Pulham, C. R.; Downs, A. J.; Goode, M. J.; Rankin, D. W. H.; Robertson, H. E. Gallane: Synthesis, Physical and Chemical Properties, and Structure of the Gaseous Molecule $\mathrm{Ga}_{2} \mathrm{H}_{6}$ As Determined by Electron Diffraction. J. Am. Chem. Soc. 1991, 113, 5149-5162.

243. Downs, A. J.; Pulham, C. R. The Hunting of the Gallium Hydrides. Adv. Inorg. Chem. 1994, $41,171-232$.

244. Gao, G.; Wang, H.; Bergara, A.; et al. Metallic and Superconducting Gallane under Pressure. Phys. Rev. B 2011, 84, 064118.

245. Szczęśniak, R.; Durajski, A. P. Superconducting State above the Boiling Point of Liquid Nitrogen in the $\mathrm{GaH}_{3}$ Compound. Supercond. Sci. Technol. 2014, 27, 015003.

246. Downs, A. J.; Pulham, C. R. The Hydrides of Aluminum, Gallium, Indium, Thallium: A Re-Evaluation. Chem. Soc. Rev. 1994, 23, 175-184.

247. Hunt, P.; Schwerdtfeger, P. Are the Compounds $\mathrm{InH}_{3}$ and $\mathrm{TlH}_{3}$ Stable Gas Phase or Solid State Species? Inorg. Chem. 1996, 35, 2085-2088. 
248. Andrews, L.; Wang, X. Infrared Spectra of Indium Hydrides in Solid Hydrogen and of Solid Indane. Angew. Chem. 2004, 116, 1738-1741.

249. Wang, X.; Andrews, L. Infrared Spectra of Indium Hydrides in Solid Hydrogen and Neon. J. Phys. Chem. A 2004, 108, 4440-4448.

250. Wang, X.; Andrews, L. Infrared Spectra of Thallium Hydrides in Solid Neon, Hydrogen, and Argon. J. Phys. Chem. A 2004, 108, 3396-3402.

251. Liu, Y.; Duan, D.; Tian, F.; et al. Pressure-Induced Structures and Properties in Indium Hydrides. Inorg. Chem. 2015, 54, 9924-9928.

252. Chen, P.-N.; Zha, C.-S.; Chen, X.-J.; et al. Raman Study of Phase Transitions in Compressed Methane using Moissanite Anvil Cells. Phys. Rev. B 2011, 84, 104110.

253. Somayazulu, M. S.; Finger, L. W.; Hemley, R. J.; Mao, H. K. High-Pressure Compounds in Methane-Hydrogen Mixtures. Science 1996, 271, 1400-1402.

254. Martínez-Canales, M.; Bergara, A. No Evidence of Metallic Methane at High Pressure. High Press. Res. 2006, 26, 369-375.

255. Gao, G.; Oganov, A. R.; Ma, Y.; et al. Dissociation of Methane under High Pressure. J. Chem. Phys. 2010, 133, 144508.

256. Zhao, J.; Feng, W.-X.; Liu, Z.-M.; et al. Structural Investigation of Solid Methane at High Pressure. Chin. Phys. Lett. 2010, 27, 066101.

257. Lin, H.; Li, Y.-1.; Zeng, Z.; Chen, X.-j.; Lin, H. Q. Structural, Electronic, and Dynamical Properties of Methane under High Pressure. J. Chem. Phys. 2011, 134, 064515.

258. Liu, H.; Naumov, I. I.; Hemley, R. J. Dense Hydrocarbon Structures at Megabar Pressures. J. Phys. Chem. Lett. 2016, 7, 4218-4222.

259. Liu, Y.; Duan, D.; Tian, F.; et al. Crystal Structures and Properties of the $\mathrm{CH}_{4} \mathrm{H}_{2}$ Compound under High Pressure. RSC Adv. 2014, 4, 37569-37574.

260. Yao, Y.; Tse, J. S.; Ma, Y.; Tanaka, K. Superconductivity in High-Pressure $\mathrm{SiH}_{4}$. EPL 2007, $78,37003$.

261. Degtyareva, O.; Martínez-Canales, M.; Bergara, A.; Chen, X.-J.; Song, Y. et al. Crystal Structure of $\mathrm{SiH}_{4}$ at High Pressure. Phys. Rev. B 2007, 76, 064123.

262. Hanfland, M.; Proctor, J. E.; Guillaume, C. L.; Degtyareva, O.; Gregoryanz, E. HighPressure Synthesis, Amorphization, and Decomposition of Silane. Phys. Rev. Lett. 2011, $106,095503$.

263. Kurzydłowski, D.; Grochala, W. Phonon Dispersion Analysis as an Indispensable Tool for Predictions of Solid State Polymorphism and Dynamic Metastability: Case of Compressed Silane. Acta Phys. Pol. A 2011, 119, 895-900. 
264. Zhang, H.; Jin, X.; Lv, Y.; et al. High-Temperature Superconductivity in Compressed Solid Silane. Sci. Rep. 2015, 5, 8845.

265. Cui, W.; Shi, J.; Liu, H.; et al. Hydrogen Segregation and Its Roles in Structural Stability and Metallization: Silane under Pressure. Sci. Rep. 2015, 5, 13039.

266. Sun, L.; Ruoff, A. L.; Zha, C.-S.; Stupian, G. High Pressure Studies on Silane to 210 GPa at $300 \mathrm{~K}$ : Optical Evidence of an Insulator-Semiconductor Transition. J. Phys.: Condens. Matter 2006, 18, 8573-8580.

267. Strobel, T. A.; Goncharov, A. F.; Seagle, C. T.; Liu, Z.; Somayazulu, M. et al. High-Pressure Study of Silane to 150 GPa. Phys. Rev. B 2011, 83, 144102.

268. Chen, X.-J.; Struzhkin, V. V.; Song, Y.; Goncharov, A. F.; Ahart, M. et al. Pressure-Induced Metallization of Silane. Proc. Natl. Acad. Sci. U.S.A. 2008, 105, 20-23.

269. Chen, X.-J.; Wang, J.-L.; Struzhkin, V. V.; Mao, H.-k.; Hemley, R. J. et al. Superconducting Behavior in Compressed Solid $\mathrm{SiH}_{4}$ with a Layered Structure. Phys. Rev. Lett. 2008, 101, 077002.

270. Kim, D. Y.; Scheicher, R. H.; Lebègue, S.; Prasongkit, J.; Arnaud, B. et al. Crystal Structure of the Pressure-Induced Metallic Phase of $\mathrm{SiH}_{4}$ from Ab Initio Theory. Proc. Natl. Acad. Sci. U.S.A. 2008, 105, 16454-16459.

271. Martínez-Canales, M.; Oganov, A. R.; Ma, Y.; Yan, Y.; Lyakhov, A. O. et al. Novel Structures and Superconductivity of Silane under Pressure. Phys. Rev. Lett. 2009, 102, 087005.

272. Yan, Y.; Gong, J.; Zhan-Guo, Z. Electronic, Vibrational, and Superconducting Properties of High-Pressure Metallic $\mathrm{SiH}_{4}$ : Ab Initio Calculations. Chin. Phys. Lett. 2010, 27, 017401.

273. Fan, W.; Wang, J.-L.; Zou, L.-J.; Zeng, Z. Non-Adiabatic Effects of Superconductor Silane under High Pressure. Chin. Phys. Lett. 2010, 27, 087402.

274. Szczęśniak, R.; Durajski, A. P. The High-Pressure Superconductivity in $\mathrm{SiH}_{4}$ : The StrongCoupling Approach. Solid State Commun. 2013, 172, 5-9.

275. Durajski, A. P.; Szczęśniak, R. Estimation of the Superconducting Parameters for Silane at High Pressure. Mod. Phys. Lett. B 2014, 28, 1450052.

276. Wang, S.; Mao, H.-k.; Chen, X.-J.; Mao, W. L. High Pressure Chemistry in the $\mathrm{H}_{2}-\mathrm{SiH}_{4}$ System. Proc. Natl. Acad. Sci. U.S.A. 2009, 106, 14763-14767.

277. Yao, Y.; Klug, D. D. Silane Plus Molecular Hydrogen as a Possible Pathway to Metallic Hydrogen. Proc. Natl. Acad. Sci. U.S.A. 2010, 107, 20893-20898.

278. Yim, W.-L.; Tse, J. S.; Iitaka, T. Pressure-Induced Intermolecular Interactions in Crystalline Silane-Hydrogen. Phys. Rev. Lett. 2010, 105, 215501. 
279. Michel, K.; Liu, Y.; Ozolins, V. Atomic Structure and $\mathrm{SiH}_{4}-\mathrm{H}_{2}$ Interactions of $\mathrm{SiH}_{4}\left(\mathrm{H}_{2}\right)_{2}$ from First Principles. Phys. Rev. B 2010, 82, 174103.

280. Shanavas, K. V.; Poswal, H. K.; Sharma, S. M. First Principles Calculations on the Effect of Pressure on $\mathrm{SiH}_{4}\left(\mathrm{H}_{2}\right)_{2}$. Solid State Commun. 2012, 152, 873-877.

281. Li, Y.; Gao, G.; Xie, Y.; et al. Superconductivity at $100 \mathrm{~K}$ in Dense $\mathrm{SiH}_{4}\left(\mathrm{H}_{2}\right)_{2}$ Predicted by First Principles. Proc. Natl. Acad. Sci. U.S.A. 2010, 107, 15708-15711.

282. Szczęśniak, R.; Durajski, A. P. On the Critical Temperature and the Energy Gap in Dense $\mathrm{SiH}_{4}\left(\mathrm{H}_{2}\right)_{2}$ at $250 \mathrm{GPa}$. Solid State Commun. 2013, 153, 26-30.

283. Durajski, A. P. Study of Thermodynamic Properties of $\mathrm{SiH}_{4}\left(\mathrm{H}_{2}\right)_{2}$ Superconductor under High Pressure. Physica C 2013, 485, 145-148.

284. Jin, X.; Meng, X.; He, Z.; et al. Superconducting High-Pressure Phases of Disilane. Proc. Natl. Acad. Sci. U.S.A 2010, 107, 9969-9973.

285. Flores-Livas, J. A.; Amsler, M.; Lenosky, T. J.; Lehtovaara, L.; Botti, S. et al. High-Pressure Structures of Disilane and Their Superconducting Properties. Phys. Rev. Lett. 2012, 108, 117004.

286. Martínez-Canales, M.; Bergara, A.; Feng, J.; Grochala, W. Pressure Induced Metallization of Germane. J. Phys. Chem. Solids 2006, 67, 2095-2099.

287. Li, Z.; Yu, W.; Jin, C. First-Principles Calculation on Phase Stability and Metallization in $\mathrm{GeH}_{4}$ under Pressure. Solid State Commun. 2007, 143, 353-357.

288. Gao, G.; Oganov, A. R.; Bergara, A.; Martínez-Canales, M.; Cui, T. et al. Superconducting High Pressure Phase of Germane. Phys. Rev. Lett. 2008, 101, 107002.

289. Szczęśniak, R.; Durajski, A. P. Detailed Study of the Superconducting Properties in Compressed Germane. Eur. Phys. J. B 2015, 88, 342.

290. Zhang, C.; Chen, X.-J.; Li, Y.-L.; et al. Structural Transitions of Solid Germane under Pressure. EPL 2010, 90, 66006.

291. Zhang, C.; Chen, X.-J.; Li, Y.-L.; Struzhkin, V. V.; Hemley, R. J. et al. Superconductivity in Hydrogen-Rich Material: $\mathrm{GeH}_{4}$. J. Supercond. Nov. Magn. 2010, 23, 717-719.

292. Szczęśniak, R.; Durajski, A. P.; Szczęśniak, D. Study of the Superconducting State in the Cmmm Phase of $\mathrm{GeH}_{4}$ compound. Solid State Commun. 2013, 165, 39-44.

293. Zhang, H.; Jin, X.; Lv, Y.; et al. Investigation of Stable Germane Structures under HighPressure. Phys. Chem. Chem. Phys. 2015, 17, 27630-27635.

294. Zhong, G.; Zhang, C.; Chen, X.; et al. Structural, Electronic, Dynamical, and Superconducting Properties in Dense $\mathrm{GeH}_{4}\left(\mathrm{H}_{2}\right)_{2}$. J. Phys. Chem. C 2012, 116, 5225-5234. 
295. Zhang, H.; Jin, X.; Lv, Y.; et al. A Novel Stable Hydrogen Rich $\mathrm{SnH}_{8}$ Under High Pressure. RSC Adv. 2015, 5, 107637-107641.

296. Cheng, Y.; Zhang, C.; Wang, T.; et al. Pressure-Induced Superconductivity in $\mathrm{H}_{2}-$ Containing Hydride $\mathrm{PbH}_{4}\left(\mathrm{H}_{2}\right)_{2}$. Sci. Rep. 2015, 5, 16475.

297. Strobel, T. A.; Chen, X.-J.; Somayazulu, M.; Hemley, R. J. Vibrational Dynamics, Intermolecular Interactions, and Compound Formation in $\mathrm{GeH}_{4}-\mathrm{H}_{2}$ under Pressure. J. Chem. Phys. 2010, 133, 164512.

298. Szczęśniak, R.; Szczęśniak, D.; Durajski, A. P. Thermodynamics of the Superconducting Phase in Compressed $\mathrm{GeH}_{4}\left(\mathrm{H}_{2}\right)_{2}$. Solid State Commun. 2014, 184, 6-11.

299. Zhong, G.; Zhang, C.; Wu, G.; et al. Superconductivity in $\mathrm{GeH}_{4}\left(\mathrm{H}_{2}\right)_{2}$ above $220 \mathrm{GPa}$ HighPressure. Physica B 2013, 410, 90-92.

300. Hou, P.; Tian, F.; Li, D.; et al. Ab Initio Study of Germanium-Hydride Compounds under High Pressure. RSC Adv. 2015, 5, 19432-19438.

301. Esfahani, M. M. D.; Oganov, A. R.; Niu, H.; Zhang, J. Superconductivity and Unexpected Chemistry of Germanium Hydrides under Pressure. Phys. Rev. B 2017, 95, 134506.

302. Abe, K.; Ashcroft, N. Quantum Disproportionation: The High Hydrides at Elevated Pressures. Phys. Rev. B 2013, 88, 174110.

303. Tse, J. S.; Yao, Y.; Tanaka, K. Novel Superconductivity in Metallic $\mathrm{SnH}_{4}$ under High Pressure. Phys. Rev. Lett. 2007, 98, 117004.

304. Gao, G.; Oganov, A. R.; Li, P.; et al. High-Pressure Crystal Structures and Superconductivity of Stannane $\left(\mathrm{SnH}_{4}\right)$. Proc. Natl. Acad. Sci. U.S.A. 2010, 107, 1317-1320.

305. Zhang, H.; Jin, X.; Lv, Y.; et al. Pressure-Induced Phase Transition of $\mathrm{SnH}_{4}$ : A New Layered Structure. RSC Adv. 2016, 6, 10456-10461.

306. Gonzalez-Morelos, P.; Hoffmann, R.; Ashcroft, N. W. Segregation into Layers: a General Problem for Structural Instability under Pressure, Exemplified by $\mathrm{SnH}_{4}$. ChemPhysChem 2010, 11, 3105-3112.

307. Esfahani, M. M. D.; Wang, Z.; Oganov, A. R.; et al. Superconductivity of Novel Tin Hydrides $\left(\mathrm{Sn}_{n} \mathrm{H}_{m}\right)$ under Pressure. Sci. Rep. 2016, 6, 22873.

308. Wang, X.; Andrews, L. Infrared Spectra of Group 14 Hydrides in Solid Hydrogen: Experimental Observation of $\mathrm{PbH}_{4}, \mathrm{~Pb}_{2} \mathrm{H}_{2}$, and $\mathrm{Pb}_{2} \mathrm{H}_{4}$. J. Am. Chem. Soc. 2003, 125, 6581-6587.

309. Zaleski-Ejgierd, P.; Hoffmann, R.; Ashcroft, N. W. High Pressure Stabilization and Emergent Forms of $\mathrm{PbH}_{4}$. Phys. Rev. Lett. 2011, 107, 037002.

310. Hewat, A. W.; Riekel, C. The Crystal Structure of Deuteroammonia between 2 and $180 \mathrm{~K}$ by Neutron Powder Profile Refinement. Acta Cryst. 1979, A35, 569-571. 
311. Loveday, J. S.; Nelmes, R. J.; Marshall, W. G.; Besson, J. M.; Klotz, S. et al. Structure of Deuterated Ammonia IV. Phys. Rev. Lett. 1996, 76, 74-77.

312. Datchi, F.; Ninet, S.; Gauthier, M.; et al. Solid Ammonia at High Pressure: A Single-Crystal X-Ray Diffraction Study to 123 GPa. Phys. Rev. B 2006, 73, 174111.

313. Gauthier, M.; Pruzan, P.; Chervin, J. C.; Besson, J. M. Raman Scattering Study of Ammonia up to 75 GPa: Evidence for Bond Symmetrization at 60 GPa. Phys. Rev. B 1988, 37, 2102 2115.

314. Fortes, A. D.; Brodholt, J. P.; Wood, I. G.; Vočadlo, L. Hydrogen Bonding in Solid Ammonia from Ab Initio Calculations. J. Chem. Phys. 2003, 118, 5987-5994.

315. Pickard, C. J.; Needs, R. J. Highly Compressed Ammonia Forms an Ionic Crystal. Nat. Mater. 2008, 7, 775-779.

316. Ninet, S.; Datchi, F.; Dumas, P.; et al. Experimental and Theoretical Evidence for an Ionic Crystal of Ammonia at High Pressure. Phys. Rev. B 2014, 89, 174103.

317. Spaulding, D. K.; Weck, G.; Loubeyre, P.; et al. Pressure-Induced Chemistry in a NitrogenHydrogen Host-Guest Structure. Nat. Commun. 2014, 5, 5739.

318. Goncharov, A. F.; Holtgrewe, N.; Qian, G.; et al. Backbone $\mathrm{N}_{x} \mathrm{H}$ Compounds at High Pressures. J. Chem. Phys. 2015, 142, 214308.

319. Chidester, B. A.; Strobel, T. A. The Ammonia-Hydrogen System under Pressure. J. Phys. Chem. A 2011, 115, 10433-10437.

320. Drozdov, A. P.; Eremets, M. I.; Troyan, I. A. Superconductivity above $100 \mathrm{~K}$ in $\mathrm{PH}_{3}$ at High Pressures. arXiv:1508.06224

321. Flores-Livas, J. A.; Amsler, M.; Heil, C.; et al. Superconductivity in Metastable Phases of Phosphorus-Hydride Compounds under High Pressure. Phys. Rev. B 2016, 93, 020508(R).

322. Shamp, A.; Terpstra, T.; Bi, T.; et al. Decomposition Products of Phosphane Under Pressure: $\mathrm{PH}_{2}$ Stable and Superconducting? J. Am. Chem. Soc. 2016, 138, 1884-1892.

323. Liu, H.; Li, Y.; Gao, G.; Tse, J. S.; Naumov, I. I. Crystal Structure and Superconductivity of $\mathrm{PH}_{3}$ at High Pressures. J. Phys. Chem. C 2016, 120, 3458-3461.

324. Durajski, A. P. Quantitative Analysis of Nonadiabatic Effects in Dense $\mathrm{H}_{3} \mathrm{~S}$ and $\mathrm{PH}_{3}$ Superconductors. Sci. Rep. 2016, 6, 38570.

325. Bi, T.; Miller, D. P.; Shamp, A.; Zurek, E. Superconducting Phases of Phosphorus Hydride under Pressure: Stabilization by Mobile Molecular Hydrogen. Angew. Chem. 2017, 129, $10326-10329$.

326. Fu, Y.; Du, X.; Zhang, L.; et al. High-Pressure Phase Stability and Superconductivity of Pnictogen Hydrides and Chemical Trends for Compressed Hydrides. Chem. Mater. 2016, 28, 1746-1755. 
327. Ma, Y.; Duan, D.; Li, D.; et al. The Unexpected Binding and Superconductivity in $\mathrm{SbH}_{4}$ at High Pressure. arXiv:1506.03889v2.

328. Abe, K.; Ashcroft, N. W. Stabilization and Highly Metallic Properties of Heavy Group-V Hydrides at High Pressures. Phys. Rev. B. 2015, 92, 224109.

329. Ma, Y.; Duan, D.; Li, D.; et al. High-Pressure Structures and Superconductivity of Bismuth Hydrides. arXiv:1511.05291v1.

330. Petrenko, V. F.; Whitworth, R. W. Physics of Ice; Oxford University Press, Oxford, 1999.

331. Loubeyre, P.; LeToullec, R.; Wolanin, E.; Hanfland, M.; Hausermann, D. Modulated Phases and Proton Centring in Ice Observed by X-ray Diffraction up to 170 GPa. Nature 1999, 397, 503-506.

332. Benoit, M.; Bernasconi, M.; Focher, P.; Parrinello, M. New High-Pressure Phase of Ice. Phys. Rev. Lett. 1996, 76, 2934-2936.

333. Militzer, B.; Wilson, H. F. New Phases of Water Ice Predicted at Megabar Pressures. Phys. Rev. Lett. 2010, 105, 195701.

334. Wang, Y.; Liu, H.; Lv, J.; et al. High Pressure Partially Ionic Phase of Water Ice. Nat. Commun. 2011, 2, 563.

335. Hermann, A.; Ashcroft, N. W.; Hoffmann, R. High Pressure Ices. Proc. Natl. Acad. Sci. U.S.A. 2012, 109, 745-750.

336. Ji, M.; Umemoto, K.; Wang, C. Z.; Ho, K. M.; Wentzcovitch, R. M. Ultrahigh-Pressure Phases of $\mathrm{H}_{2} \mathrm{O}$ Ice Predicted Using an Adaptive Genetic Algorithm. Phys. Rev. B 2011, 84, 220105(R).

337. McMahon, J. M. Ground-State Structures of Ice at High Pressures from AbInitio Random Structure Searching. Phys. Rev. B 2011, 84, 220104(R) (1-4).

338. Vos, W. L.; Finger, L. W.; Hemley, R. J.; Mao, H. K. Novel $\mathrm{H}_{2}-\mathrm{H}_{2} \mathrm{O}$ Clathrates at High Pressures. Phys. Rev. Lett. 1993, 71, 3150-3153.

339. Qian, G. R.; Lyakhov, A. O.; Zhu, Q.; Oganov, A. R.; Dong, X. Novel Hydrogen Hydrate Structures under Pressure. Sci. Rep. 2014, 4, 5606.

340. Pickard, C. J.; Martínez-Canales, M.; Needs, R. J. Decomposition and Terapascal Phases of Water Ice. Phys. Rev. Lett. 2013, 110, 245701.

341. Zhang, S.; Wilson, H. F.; Driver, K. P.; Militzer, B. $\mathrm{H}_{4} \mathrm{O}$ and Other Hydrogen-Oxygen Compounds at Giant-Planet Core Pressures. Phys. Rev. B 2013, 87, 024112.

342. Shimizu, H.; Murashima, H.; Sasaki, S. High-Pressure Raman Study of Solid Deuterium Sulfide up to 17 GPa. J. Chem. Phys. 1992, 97, 7137-7139. 
343. Endo, S.; Ichimiya, N.; Koto, K.; Sasaki, S.; Shimizu, H. X-ray-Diffraction Study of Solid Hydrogen Sulfide under High Pressure. Phys. Rev. B 1994, 50, 5865-5867.

344. Shimizu, H.; Yamaguchi, H.; Sasaki, S.; et al. Pressure-Temperature Phase Diagram of Solid Hydrogen Sulfide Determined by Raman Spectroscopy. Phys. Rev. B 1995, 51, 9391(R)-9394(R).

345. Loveday, J. S.; Nelmes, R. J.; Marshall, W. G.; Besson, J. M.; Klotz, S. et al. Structural Studies of Ices at High Pressure. Physica B 1997, 241, 240-246.

346. Fujihisa, H.; Yamawaki, H.; Sakashita, M.; et al. Structures of $\mathrm{H}_{2} \mathrm{~S}$ : Phases I and IV under High Pressure. Phys. Rev. B 1998, 57, 2651-2654.

347. Endo, S.; Honda, A.; Koto, K.; et al. Crystal Structure of High-Pressure Phase-IV Solid Hydrogen Sulfide. Phys. Rev. B 1998, 57, 5699-5703.

348. Endo, S.; Honda, A.; Sasaki, S.; et al. High-Pressure Phase of Solid Hydrogen Sulfide. Phys. Rev. B 1996, 54, R717(R)-R719(R).

349. Shimizu, H.; Ushida, T.; Sasaki, S.; et al. High-Pressure Phase Transitions of Solid $\mathrm{H}_{2} \mathrm{~S}$ Probed by Fourier-Transform Infrared Spectroscopy. Phys. Rev. B 1997, 55, 5538-5541.

350. Sakashita, M.; Yamawaki, H.; Fujihisa, H.; et al. Pressure-Induced Molecular Dissociation and Metallization in Hydrogen-Bonded $\mathrm{H}_{2} \mathrm{~S}$ Solid. Phys. Rev. Lett. 1997, 79, 1082-1085.

351. Rousseau, R.; Boero, M.; Bernasconi, M.; Parrinello, M.; Terakura, K. Static Structure and Dynamical Correlations in High Pressure $\mathrm{H}_{2}$ S. Phys. Rev. Lett. 1999, 83, 2218-2221.

352. Shimizu, H.; Nakamichi, Y.; Sasaki, S. Pressure Induced Phase Transition in Solid Hydrogen Sulphide at 11 GPa. J. Chem. Phys. 1991, 95, 2036-2040.

353. Rousseau, R.; Boero, M.; Bernasconi, M.; Parrinello, M.; Terakura, K. AbInitio Simulation of Phase Transitions and Dissociation of $\mathrm{H}_{2} \mathrm{~S}$ at High Pressure. Phys. Rev. Lett. 2000, 85, 1254-1257.

354. Wang, L.; He, Z.; Ma, Y.; et al. Structural and Dynamical Properties of $\mathrm{H}_{2} \mathrm{~S}$ under High Pressure: Ab Initio Molecular Dynamics Studies. J. Phys. Condens. Matter 2007, 19, 425232.

355. Wang, L.; Tian, F.; Feng, W.; Chen, C.; He, Z. Order-Disorder Phase Transition and Dissociation of Hydrogen Sulfide under High pressure: Ab Initio Molecular Dynamics Study. J. Chem. Phys. 2010, 132, 164506.

356. Li, Y.; Hao, J.; Liu, H.; Li, Y.; Ma, Y. The Metallization and Superconductivity of Dense Hydrogen Sulfide. J. Chem. Phys. 2014, 140, 174712.

357. Durajski, A. P.; Szczęśniak, R.; Li, Y. Non-BCS thermodynamic properties of $\mathrm{H}_{2} \mathrm{~S}$ superconductor. Physica C 2015, 515, 1-6. 
358. Strobel, T. A.; Ganesh, P.; Somayazulu, M.; Kent, P. R. C.; Hemley, R. J. Novel Cooperative Interactions and Structural Ordering in $\mathrm{H}_{2} \mathrm{~S}-\mathrm{H}_{2}$. Phys. Rev. Lett. 2011, 107, 255503.

359. Duan, D.; Liu, Y.; Tian, F.; et al. Pressure-Induced Metallization of Dense $\left(\mathrm{H}_{2} \mathrm{~S}\right)_{2} \mathrm{H}_{2}$ with high-T ${ }_{c}$ Superconductivity. Sci. Rep. 2014, 4, 6968.

360. Einaga, M.; Sakata, M.; Ishikawa, T.; Shimizu, K.; Eremets, M. I. et al. Crystal Structure of 200 K-Superconducting Phase of Sulfur Hydride System. Nat. Phys. 2016, 12, 835-838.

361. Troyan, I.; Gavriliuk, A.; Rüffer, R.; Chumakov, A.; Mironovich, A. et al. Observation of Superconductivity in Hydrogen Sulfide from Nuclear Resonant Scattering. Science 2016, 351, 1303-1306.

362. Capitani, F.; Langerome, B.; Brubach, J. B.; Roy, P.; Drozdov, A. et al. Spectroscopic Evidence of a New Energy Scale for Superconductivity in $\mathrm{H}_{3} \mathrm{~S}$. Nat. Phys. 2017, 13, 859-863.

363. Goncharov, A. F.; Lobanov, S. S.; Prakapenka, V. B.; Greenberg, E. Stable High-Pressure Phases in the HS System Determined by Chemically Reacting Hydrogen and Sulfur. Phys. Rev. B 2017, 95, 140101(R).

364. Guigue, B.; Marizy, A.; Loubeyre, P. Direct Synthesis of Pure $\mathrm{H}_{3} \mathrm{~S}$ from $\mathrm{S}$ and H Elements: No Evidence of the Cubic Superconducting Phase up to 160 GPa. Phys. Rev. B 2017, 95, 020104(R).

365. Li, Y.; Wang, L.; Liu, H.; et al. Dissociation Products and Structures of Solid $\mathrm{H}_{2} \mathrm{~S}$ at Strong Dompression. Phys. Rev. B 2016, 93, 020103(R).

366. Ishikawa, T.; Nakanishi, A.; Shimizu, K.; Katayama-Yoshida, H.; Oda, T. et al. Superconducting $\mathrm{H}_{5} \mathrm{~S}_{2}$ Phase in Sulfur-Hydrogen System under High-Pressure. Sci. Rep. 2016, 6, 23160.

367. Flores-Livas, J. A.; Sanna, A.; Gross, E. K. U. High Temperature Superconductivity in Sulfur and Selenium Hydrides at High Pressure. Eur. Phys. J. B. 2016, 89, 1-6.

368. Papaconstantopoulos, D. A.; Klein, B. M.; Mehl, M. J.; Pickett, W. E. Cubic $\mathrm{H}_{3} \mathrm{~S}$ around 200 GPa: An Atomic Hydrogen Superconductor Stabilized by Sulfur. Phys. Rev. B 2015, $91,184511$.

369. Bernstein, N.; Hellberg, C. S.; Johannes, M. D.; Mazin, I. I.; Mehl, M. J. What Superconducts in Sulfur Hydrides under Pressure and Why. Phys. Rev. B 2015, 91, 060511(R).

370. Duan, D.; Huang, X.; Tian, F.; et al. Pressure-Induced Decomposition of Solid Hydrogen Sulfide. Phys. Rev. B 2015, 91, 180502(R).

371. Errea, I.; Calandra, M.; Pickard, C. J.; Nelson, J.; Needs, R. J. et al. Hydrogen Sulphide at High Pressure: a Strongly-Anharmonic Phonon-Mediated Superconductor. Phys. Rev. Lett. 2015, 114, 157004. 
372. Akashi, R.; Kawamura, M.; Tsuneyuki, S.; Nomura, Y.; Arita, R. First-Principles Study of the Pressure and Crystal-Structure Dependences of the Superconducting Transition Temperature in Compressed Sulfur Hydrides. Phys. Rev. B 2015, 91, 224513.

373. Errea, I.; Calandra, M.; Pickard, C. J.; Nelson, J. R.; Needs, R. J. et al. Quantum HydrogenBond Symmetrization in the Superconducting Hydrogen Sulfide System. Nature 2016, 532, 81-84.

374. Quan, Y.; Pickett, W. E. Van Hove Singularities and Spectral Smearing in HighTemperature Superconducting $\mathrm{H}_{3}$ S. Phys. Rev. B 2016, 93, 104526.

375. Sano, W.; Koretsune, T.; Tadano, T.; Akashi, R.; Arita, R. Effect of Van Hove Singularities on High- $\mathrm{T}_{c}$ Superconductivity in $\mathrm{H}_{3}$ S. Phys. Rev. B 2016, 93, 094525.

376. Ortenzi, L.; Cappelluti, E.; Pietronero, L. Band Structure and Electron-Phonon Coupling in $\mathrm{H}_{3} \mathrm{~S}$ : A Tight-Binding Model. Phys. Rev. B 2016, 94, 064507.

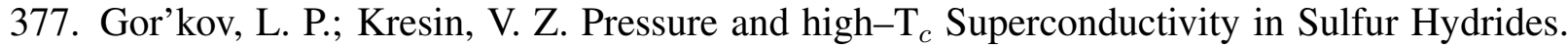
Sci. Rep. 2016, 6, 25608.

378. Goncharov, A. F.; Lobanov, S. S.; Kruglov, I.; Zhao, X. M.; Chen, X. J. et al. Hydrogen Sulfide at High Pressure: Change in Stoichiometry. Phys. Rev. B 2016, 93, 174105.

379. Bussmann-Holder, A.; Köhler, J.; Simon, A.; Whangbo, M.; Bianconi, A. Multigap Superconductivity at Extremely High Temperature: A Model for the Case of Pressurized $\mathrm{H}_{2} \mathrm{~S}$. $J$. Supercond. Nov. Magn. 2017, 30, 151-156.

380. Jarlborg, T.; Bianconi, A. Breakdown of the Migdal approximation at Lifshitz transitions with giant zero-point motion in the $\mathrm{H}_{3} \mathrm{~S}$ superconductor. Sci. Rep. 2016, 6, 24816 (1-12).

381. Szczęśniak, R.; Durajski, A. P. The Isotope Effect in $\mathrm{H}_{3} \mathrm{~S}$ Superconductor. Solid State Commun. 2017, 249, 30-33.

382. Azadi, S.; Kühne, T. D. High-Pressure Hydrogen Sulfide by Diffusion Quantum Monte Carlo. J. Chem. Phys. 2017, 146, 084503.

383. Arita, R.; Koretsune, T.; Sakai, S.; et al. Nonempirical Calculation of Superconducting Transition Temperatures in Light-Element Superconductors. Adv. Mater. 2017, 29, 1602421.

384. Gordon, E. E.; Xu, K.; Xiang, H.; Bussmann-Holder, A.; Kremer, R. K. et al. Structure and Composition of the $200 \mathrm{~K}$-Superconducting Phase of $\mathrm{H}_{2} \mathrm{~S}$ at Ultrahigh Pressure: The Perovskite $\left(\mathrm{SH}^{-}\right)\left(\mathrm{H}_{3} \mathrm{~S}^{+}\right)$. Angew. Chem. Int. Ed. 2016, 55, 3682-3684.

385. Majumdar, A.; Tse, J. S.; Yao, Y. Modulated Structure Calculated for Superconducting Hydrogen Sulfide. Angew. Chem. 2017, 129, 11548-11551.

386. Akashi, R.; Sano, W.; Arita, R.; Tsuneyuki, S. Possible "Magneli" Phases and SelfAlloying in the Superconducting Sulfur Hydride. Phys. Rev. Lett. 2016, 117, 075503. 
387. Heil, C.; Boeri, L. Influence of Bonding on Superconductivity in High-Pressure Hydrides. Phys. Rev. B 2015, 92, 060508(R).

388. Ge, Y.; Zhang, F.; Yao, Y. First-Principles Demonstration of Superconductivity at $280 \mathrm{~K}$ in Hydrogen Sulfide with Low Phosphorus Substitution. Phys. Rev. B 2016, 93, 224513.

389. Hirsch, J. E.; Marsiglio, F. Hole Superconductivity in $\mathrm{H}_{2} \mathrm{~S}$ and Other Sulfides under High Pressure. Physica C 2015, 511, 45-49.

390. Zhang, S.; Wang, Y.; Zhang, J.; et al. Phase Diagram and High-Temperature Superconductivity of Compressed Selenium Hydrides. Sci. Rep. 2015, 5, 15433.

391. Pace, E. J.; Binns, J.; Peña Alvarez, M.; Dalladay-Simpson, P. Synthesis and Stability of Hydrogen Selenide Compounds at High Pressure. J. Chem. Phys. 2017, 147, 184303.

392. Zhang, X.; Xu, W.; Wang, Y.; et al. Synthesis and Properties of Selenium Trihydride at High Pressures. Phys. Rev. B 2018, 97, 064107.

393. Zhong, X.; Wang, H.; Zhang, J.; et al. Tellurium Hydrides at High Pressures: HighTemperature Superconductors. Phys. Rev. Lett. 2016, 116, 057002.

394. Liu, Y.; Duan, D.; Tian, F.; et al. Prediction of Stoichiometric $\mathrm{PoH}_{n} \mathrm{Compounds}_{\text {: Crystal }}$ Structures and Properties. RSC Adv. 2015, 5, 103445-103450.

395. Johnson, M. W.; Sándor, E.; Arzi, E. The Crystal Structure of Deuterium Fluoride. Acta Cryst. 1975, B31, 1998-2003.

396. Sándor, E.; Farrow, R. F. C. Crystal Structure of Solid Hydrogen Chloride and Deuterium Chloride. Nature 1967, 213, 171-172.

397. Ikram, A.; Torrie, B. H.; Powell, B. M. Structures of Solid Deuterium Bromide and Deuterium Iodide. Mol. Phys. 1993, 79, 1037-1049.

398. Pinnick, D. A.; Katz, A. I.; Hanson, R. C. Pressure Effects on Raman Scattering from Solid HF and DF. Phys. Rev. B 1989, 39, 8677-8688.

399. Katoh, E.; Yamawaki, H.; Fujihisa, H.; Sakashita, M.; Aoki, K. Raman and Infrared Study of Phase Transitions in Solid HBr under Pressure. Phys. Rev. B 1999, 59, 11244-11250.

400. Aoki, K.; Katoh, E.; Yamawaki, H.; Sakashita, M.; Fujihisa, H. Hydrogen-Bond Symmetrization and Molecular Dissociation in Hydrogen Halids. Physica B 1999, 265, 83-86.

401. Ikeda, T.; Sprik, M.; Terakura, K.; Parrinello, M. Pressure-Induced Structural and Chemical Changes of Solid HBr. J. Chem. Phys. 1999, 111, 1595-1607.

402. Sode, O.; Hirata, S. Second-Order Many-Body Perturbation Study of Solid Hydrogen Fluoride under Pressure. Phys. Chem. Chem. Phys. 2012, 14, 7765-7779.

403. Zhang, L.; Wang, Y.; Zhang, X.; Ma, Y. High-Pressure Phase Transitions of Solid HF, HCl, and HBr: An Ab Initio Evolutionary Study. Phys. Rev. B 2010, 82, 014108. 
404. Duan, D.; Tian, F.; He, Z.; et al. Hydrogen Bond Symmetrization and Superconducting Phase of $\mathrm{HBr}$ and $\mathrm{HCl}$ under High Pressure: An Ab Initio Study. J. Chem. Phys. 2010, 133, 074509.

405. Chen, C.; Xu, Y.; Sun, X.; Wang, S. Novel Superconducting Phases of $\mathrm{HCl}$ and $\mathrm{HBr}$ under High Pressure: An Ab Initio Study. J. Phys. Chem. C 2015, 119, 17039-17043.

406. Lu, S.; Wu, M.; Liu, H.; Tse, J. S.; Yang, B. Prediction of Novel Crystal Structures and Superconductivity of Compressed HBr. RSC Adv. 2015, 5, 45812-45816.

407. Wang, Z.; Wang, H.; Tse, J. S.; Iitaka, T.; Ma, Y. Stabilization of $\mathrm{H}_{3}^{+}$in the High Pressure Crystalline Structure of $\mathrm{H}_{n} \mathrm{Cl}(\mathrm{n}=2-7)$. Chem. Sci. 2015, 6, 522-526.

408. Shamp, A.; Zurek, E. Superconducting High Pressure Phases Composed of Hydrogen and Iodine. J. Phys. Chem. Lett. 2015, 6, 4067-4072.

409. Duan, D.; Tian, F.; Liu, Y.; et al. Enhancement of $\mathrm{T}_{c}$ in the Atomic Phases of Iodine-Doped Hydrogen at High Pressures. Phys. Chem. Chem. Phys. 2015, 17, 32335-32340.

410. Duan, D.; Huang, X.; Tian, F.; et al. Predicted Formation of $\mathrm{H}_{3}^{+}$in Solid Halogen Polyhydrides at High Pressures. J. Phys. Chem. A 2015, 119, 11059-11065.

411. Zeng, Q.; Yu, S.; Li, D.; Frapper, G.; Oganov, A. R. Emergence of Novel Hydrogen Chlorides under Pressure. Phys. Chem. Chem. Phys. 2017, 19, 8236-8242.

412. Duan, D.; Tian, F.; Huang, X.; et al. arXiv:1504.01196.

413. van Straaten, J.; Silvera, I. F. Observation of Metal-Insulator and Metal-Metal Transitions in Hydrogen Iodide under Pressure. Phys. Rev. Lett 1986, 57, 766-769.

414. van Straaten, J.; Silvera, I. F. Temperature Dependence of Resistance of Solid Hydrogen Iodide at High Pressure. J. Chem. Phys. 1988, 88, 478-480.

415. Binns, J.; Dalladay-Simpson, P.; Wang, M.; Ackland, G. J.; Gregoryanz, E. et al. Formation of $\mathrm{H}_{2}$-Rich Iodine-Hydrogen Compounds at High Pressure. Phys. Rev. B 2018, 97, 024111.

416. Morales, M. A.; Schwegler, E.; Ceperley, D.; Pierleoni, C.; Hamel, S. et al. Phase Separation in Hydrogen-Helium Mixtures at Mbar Pressures. Proc. Natl. Acad. Sci. U.S.A. 2009, 106, $1324-1329$.

417. Morales, M. A.; Hamel, S.; Caspersen, K.; Schwegler, E. Hydrogen-Helium Demixing from First Principles: From Diamond Anvil Cells to Planetary Interiors. Phys. Rev. B 2013, 87,174105 .

418. Loubeyre, P.; Le Toullec, R.; Pinceaux, J.-P. Helium Compressional Effect on $\mathrm{H}_{2}$ Molecules Surrounded by Dense $\mathrm{H}_{2}-\mathrm{He}$ Mixtures. Phys. Rev. B 1985, 32, 7611-7613.

419. Barrett, C. S.; Meyer, L.; Wasserman, J. Crystal Structure of Solid Hydrogen and Deuterium, and of Neon-Hydrogen and Neon-Deuterium Mixtures. J. Chem. Phys. 1966, 45, 834-837. 
420. Loubeyre, P.; Jean-Louis, M.; LeToullec, R.; Charon-Gérard, L. High Pressure Measurements of the He-Ne Binary Phase Diagram at $296 \mathrm{~K}$ : Evidence for the Stability of a Stoichiometric $\mathrm{Ne}(\mathrm{He})_{2}$ Solid. Phys. Rev. Lett. 1993, 70, 178-181.

421. Cazorla, C.; Errandonea, D.; Sola, E. High-Pressure Phases, Vibrational Properties, and Electronic Structure of $\mathrm{Ne}(\mathrm{He})_{2}$ and $\mathrm{Ar}(\mathrm{He})_{2}$ : A First-Principles Study. Phys. Rev. B 2009, 80, 064105.

422. Loubeyre, P.; Letoullec, R.; Pinceaux, J. P. Compression of $\operatorname{Ar}\left(\mathrm{H}_{2}\right)_{2}$ up to $175 \mathrm{GPa}$ : A New Path for the Dissociation of Molecular Hydrogen? Phys. Rev. Lett. 1994, 72, 1360-1363.

423. Ji, C.; Goncharov, A. F.; Shukla, V.; et al. Stability of $\operatorname{Ar}\left(\mathrm{H}_{2}\right)_{2}$ to $358 \mathrm{GPa}$. Proc. Natl. Acad. Sci. U.S.A. 2017, 114, 3596-3600.

424. Kleppe, A. K.; Amboage, M.; Jephcoat, A. P. New High-Pressure van der Waals Compound $\mathrm{Kr}\left(\mathrm{H}_{2}\right)_{4}$ Discovered in the Krypton-Hydrogen Binary System. Sci. Rep. 2014, 4, 4989.

425. Somayazulu, M.; Dera, P.; Goncharov, A. F.; Gramsch, S. A.; Liermann, P. et al. PressureInduced Bonding and Compound Formation in Xenon-Hydrogen Solids. Nat. Chem. 2010, $2,50-53$.

426. Somayazulu, M.; Dera, P.; Smith, J.; Hemley, R. J. Structure and Stability of Solid Xe( $\left.\mathrm{H}_{2}\right)_{n}$. J. Chem. Phys. 2015, 142, 104503.

427. Chacham, H.; Koiller, B. Band-Gap Closure of $\operatorname{Ar}\left(\mathrm{H}_{2}\right)_{2}$ under Pressure. Phys. Rev. B 1995, 52, 6147-6149.

428. Bernard, S.; Loubeyre, P.; Zérah, G. Phase Transition in $\operatorname{Ar}\left(\mathrm{H}_{2}\right)_{2}$ : a Prediction of Metalic Hydrogen Organized in Lamellar Structures. Europhys. Lett. 1997, 37, 477-482.

429. Matsumoto, N.; Nagara, H. Ab Initio Calculations for High-Pressure Phases of $\operatorname{Ar}\left(\mathrm{H}_{2}\right)_{2}$. J. Phys.: Condens. Matter 2007, 19, 365237.

430. Cazorla, C.; Errandonea, D. Ab Initio Study of Compressed $\operatorname{Ar}\left(\mathrm{H}_{2}\right)_{2}$ : Structural Stability and Anomalous Melting. Phys. Rev. B. 2010, 81, 104108 (1-5).

431. Yao, Y.; Klug, D. D. High-Pressure Phase Transition and Metallization in $\operatorname{Ar}\left(\mathrm{H}_{2}\right)_{2}$. Phys. Rev. B 2011, 83, 020105(R).

432. Kaewmaraya, T.; Kim, D. Y.; Lebègue, S.; Pickard, C. J.; Needs, R. J. et al. Theoretical Investigations of Xenon-Hydrogen Solids under Pressure using Ab Initio DFT and GW Calculations. Phys. Rev. B 2011, 84, 092101.

433. Yan, X.; Chen, Y.; Kuang, X.; Xiang, S. Structure, Stability, and Superconductivity of New Xe-H Compounds under High Pressure. J. Chem. Phys. 2015, 143, 124310.

434. Tanaka, K.; Tse, J. S.; Liu, H. Electron-Phonon Coupling Mechanisms for Hydrogen-Rich Metals at High Pressure. Phys. Rev. B 2017, 96, 100502(R). 